\title{
LA CERAMICA RINVENUTA NELLA VILLA ROMANA DI SAN POTITO DI OVINDOLI (AQ)
}

\author{
DÉNES GABLER \\ Istituto di Archeologia, \\ Úri utca 49, H-1014 Budapest, Ungheria \\ E-mail: gabler.denes@btk.mta.hu
}

\begin{abstract}
Coarse pottery from deposits in the Roman villa at San Potito di Ovindoli. This paper presents the pottery originating from three groups of objects found in close association - in a well, a cistern and a burnt layer -, and the most characteristic vessel types as well as all the lamps found during the series of excavations at the site. The well excavated in the eastern part of the villa yielded pottery dating from the second half of the $\mathrm{AD} 1^{\text {st }}$ and the beginning of the $2^{\text {nd }}$ century. Only $0.5 \%$ of this ensemble was imported pottery, $6.4 \%$ was thin-walled and color-coated pottery, and $91.4 \%$ coarse pottery. In addition to these, the deposit contained a few ampullae and incense burners (turibulum). Only one piece of North-African red slip ware was found in the fill of the cistern located in the middle of the northern courtyard and the channel leading there. Here $10 \%$ of the pottery finds was composed of fine ware, and the amount of coarse pottery was significant. The fill of the well established in the later phase of the villa dated from the second half of the AD $3^{\text {rd }}$ century. However, the pottery found in it is composed of types characteristic for the AD $1^{\text {st }}$ and $2^{\text {nd }}$ centuries - if its chronology can be reconstructed at all. Amphora fragments from Hispania and a piece of samian ware form Dr. 29 from La Graufesenque was unearthed in a burnt layer in the southern part of the early villa, as well as household ware dating from the period of the Flavian dynasty and the reign of Trajan. Here fine ware constituted $7.8 \%$ of the pottery finds. Vessels that were intact or could be refit were found atop the terrazzo floor in the central part of the villa, which might have been still in use when the villa was destroyed.
\end{abstract}

Keywords: coarse pottery, deposits, Roman villa, ampullae, Samian ware from La Graufesenque, turibulum, well, cisterna

\section{INTRODUZIONE}

In passato abbiamo già pubblicato le terre sigillate italiche, (e l'unico pezzo proveniente dalla Gallia Meridionale), le sigillate africane, la ceramica comune importata dall'Africa del Nord, e le anfore rinvenute nella villa di San Potito. ${ }^{1}$

Questa volta, oltre alla pubblicazione dell'intero materiale di ceramica comune di tre contesti sigillati (Fig. 1), che, permettono di eseguire modeste analisi statistiche, si desidera presentare anche le tipologie maggiormente caratteristiche del materiale fittile rinvenuto durante le campagne di scavi eseguiti in questi ultimi venticinque anni. Pur avendo trovato questi reperti principalmente nell'humus arato o nello strato di detriti già rimossi, tuttavia

${ }^{1}$ D. GABLER: Ceramica fine rinvenuta nella villa di San Potito (AQ). ActaArchHung 62 (2011) 91-124; P. Hárshegyi: Le anfore della villa romana di San Potito .in: Ricerche archeologiche a San
Potito di Ovindoli e le aree limitrofe nell'antichità e nell'alto medioevo. (a cura di D. Gabler-F. Redő), L'Aquila 2008,133-177. 


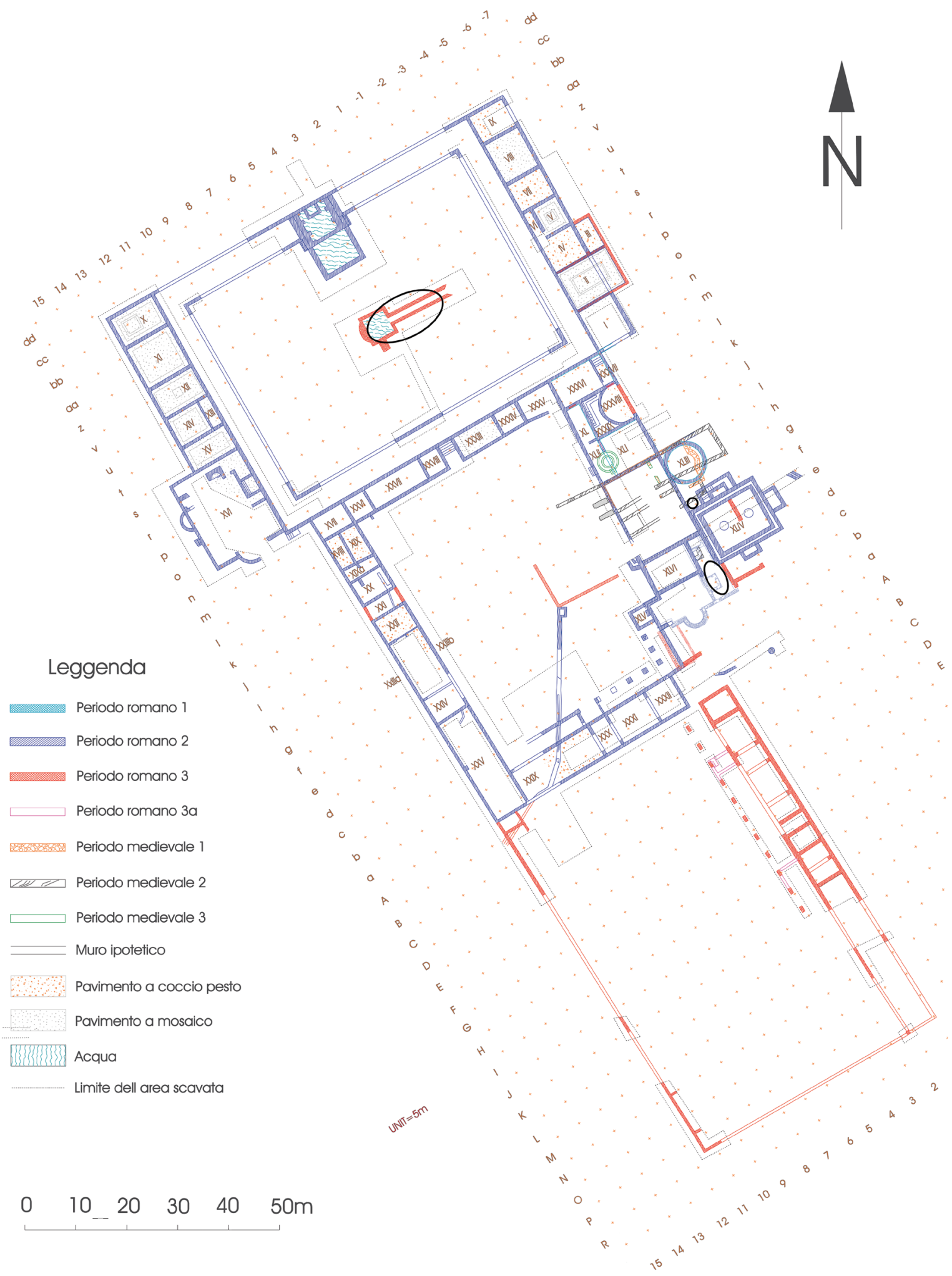

Fig. 1. Siti di rinvenimento dei complessi di reperti "chiusi” nella planimetria della villa. 
includono anche tipi che non compaiono nei contesti chiusi. Presentiamo, inoltre, anche l'intero complesso di lucerne di cui in passato abbiamo già comunicato una breve notizia².

In ogni sito di scavi la ceramica comune costituisce numericamente la proporzione preponderante del materiale venuto alla luce. Questa tipologia ceramica riveste un ruolo di rilievo nelle ricerche finalizzate a conoscere gli oggetti della vita quotidiana. In Italia fu N. Lamboglia a dare inizio a queste indagini pubblicando i materiali ritrovati ad Albintimilium e più tardi durante lo scavo della nave di Albenga ${ }^{3}$ e giunsero a maggior intensità negli anni Sessanta e Settanta del secolo passato con la pubblicazione dei reperti ritrovati a Gabii ${ }^{4}$, $\mathrm{Cosa}^{5}$, $\mathrm{Luni}^{6}$, Ostia ${ }^{7}$ e Settefinestre ${ }^{8}$. Ultimamente l'interesse di numerosi specialisti si è volto verso questo tipo di ceramica ${ }^{9}$, rendendo possibile l'elaborazione della tipologia stessa ${ }^{10}$. Prima, infatti, i reperti erano classificati secondo tre grandi categorie mentre nei volumi "ostiensi" si poterono definire anche gruppi nuovi. La classificazione della ceramica avveniva in conformità ai criteri morfologici e di funzione. Nei diversi siti si trovano tipi diversi che sono stati definiti non solo tenendo conto dell'impiego ma anche del gusto artistico, la qualità dell'argilla, il valore di mercato, nonché la relazione con il prototipo, inoltre rivestono una certa importanza anche le condizioni che resero possibile la fabbricazione locale ${ }^{11}$. Pur trattandosi di prodotti locali, tuttavia erano esposti a influenze di differente sfera d'azione, sia dal punto di vista della forma, che da quello della tecnologia adottata ${ }^{12}$. Questi prodotti erano utilizzati per il trasporto di merci differenti (vino, olio, cereali) eppure non venivano messi in circolazione come semplici contenitori, ma venivano offerti nei negozi come vasellame d'uso comune. Quest'ultima categoria è rappresentata principalmente dalla ceramica da fuoco africana che riuscì ad affermarsi nei mercati sia nell'età media dell'impero romano, che in quella tarda ${ }^{13}$. Dal punto di vista dello studio della ceramica comune la classificazione dei reperti pompeiani costituì un punto di rilievo. M. Annechino tentò di definire la funzione e la denominazione delle suppellettili fittili da fuoco di Pompei sulla base delle fonti scritte e l'iconografia. ${ }^{14}$ La rassegna e le operazioni di inventario dei reperti iniziarono solo negli anni Ottanta. Pur essendo considerata Pompei di per sé, come un sito archeologico datato, tuttavia si può definire meglio i cambiamenti avvenuti nello spettro della ceramica in base ai rilevamenti stratigrafici eseguiti nel corso degli scavi archeologici ${ }^{15}$.Di recente sono stati fatti anche tentativi finalizzati alla definizione dei luoghi di produzione sulla base di analisi archeometriche e petrografiche. ${ }^{16}$

${ }^{2}$ Zs. PONGRÁCZ: Lucerne, in: D. Gabler-F. Redő: Scavi nella villa romana di San Potito di Ovindoli (AQ). Rapporto 19891990. ActaArchHung 46 (1994) 192.

${ }^{3}$ N. LAMBOGLIA: Gli scavi di Albintimilium e la cronologia della ceramica romana. Campagne di scavi 1938-1940. Bordighera 1950; N. Lamboglia: La nave romana di Albenga. Storia e vicenda delle scoperte. Riv.Studi Liguri 18 (1952) 131-236.

${ }^{4}$ M. VeGas: Römische Keramik von Gabii (Latium). BonnerJb 168 (1968) 13-55; Eadem: Cerámica común romana del Mediterráneo Occidental. Univ. Barcelona. Publ. eventuales 22. Barcelona 1973.

${ }^{5}$ S. L. Dyson: Cosa. The Utilitarian Pottery. Mem. American Academy Rome 33, 1976.

${ }^{6}$ G. MASSARI-G. RATtI: Osservazioni sulla ceramica comune di Luni, in: Scavi di Luni II. Relazione delle campagne di Luni 1972-1974 (a cura di A. Frova) Roma 1977, 590-630.

${ }^{7}$ Ostia I. Studi miscellanei 13. Roma 1968; Ostia II. Studi miscellanei 16. Roma 1970; Ostia III. Studi miscellanei 21. Roma 1973; Ostia IV. Studi miscellanei 23, Roma 1977.

${ }^{8}$ A. RICcI: Settefinestre. Una villa schiavistica nell'Etruria romana 2. La villa e i suoi reperti (a cura di A. Ricci). Modena 1985.

${ }^{9}$ G. OLCESE: Le ceramiche comuni di Albintimilium, indagine archeologica e archeometrica sui materiali dell'area del cardine. Quad.Dipto.Archeol. e Storia delle Arti, Univ. Siena. Firenze 1993; G. Olcese: Ceramiche comuni di origine centro-meridionale tra il II secolo a.C. e il I d.C.: problemi aperti. L'evidenza dei reperti di Albintimilium. In: Les céramiques de Campanie et de Narbonnaise (Ier s. av.J.-C.- II e s. ap.J.-C.). La vaisselle de cuisine et de table. Actes des Journées d'étude (Naples 1994). Naples 1996 , 421-445.
${ }^{10}$ C. Pavolini: La ceramica comune. Le forme in Argilla depurata dell'Antiquarium. Scavi di Ostia 13. Roma 2000.

${ }^{11}$ M. BELTRÁN LLORIS: Cerámica romana, tipologia y clasificacion. Zaragoza 1978.

${ }^{12}$ G. MASSARI: Metodologia di ricerca applicata alla ceramica d'uso comune di Luni. Acta RCRF 19-20 (1979) 38-52.

${ }^{13}$ G. GASPARETTI: Produzione e consumo della ceramica comune da mensa e dispensa nella Campania romana. In: Les céramiques communes de Campanie et de Narbonnaise (1 er s.av.J.-C.-II e s. ap.J.-C). La vaisselle de cuisine et de table. Actes des Journées d' étude organisées par le Centre Jean Bérard et la Soprintendenza Archeologica per le Province di Napoli e Caserta. Naples, 27-28 mai 1994 sous la direction M-Bats. Naples 1996, 19-63.

${ }^{14}$ M. AnNECCHINO: Suppellettile fittile da cucina di Pompei, in: L'instrumentum domesticum di Ercolano e Pompei nella prima età imperiale (a cura di A. Carandini). Roma 1977, 105-120.

15 A. Gallo: La casa di Lucio Elvio Severo a Pompei. Accademia di Archeologia, Lettere e Belle Arti. Monumenti IX. Napoli 1994; S. De Caro: La villa rustica in località Villa Regina a Boscoreale. Roma 1994, 156-184.

${ }^{16}$ G. OLCESE: Le ceramiche comuni di Albintimilium. Indagine archeologica e archeometrica sui materiali dell'area del cardine. Firenze 1993; S. Santoro Bianchi-B. Fabbri: Il contributo delle analisi archeometriche allo studio delle ceramiche grezze e comuni. Il rapporto forma/funzione/impasto. Atti della I giornata di archeometria della ceramica. (a cura di S. Santoro Bianchi) Studi e scavi 4. Bologna 1997. 
Del resto, questa merce, è presente nella bibliografia del tema sotto diverse denominazioni, e la loro indicazione rimane coerente solo negli idiomi neolatini. ${ }^{17}$ Per ceramica comune praticamente s'intende un tipo di ceramica priva di ornamenti, non pregiata, perciò è più difficile individuare le officine in cui fu fabbricata ed è, naturalmente, anche meno vistosa. I ricercatori, anche nel caso di un solo luogo di fabbricazione, trattano separatamente la cosiddetta ceramica fine da quella da fuoco, e d'altronde, proprio nel caso della ceramica a pareti sottili, si osservano chiaramente le difficoltà di individuare una linea netta che divida le due tipologie fittili e così nel corso delle ricerche, la denominazione di ceramica comune copre tipologie di funzione, luogo di fabbricazione, uso e data di produzione molto. Generalmente sono distinte le tipologie della ceramica da mensa e da dispensa (brocche, coppe, coperchi) e della ceramica da fuoco (tegame, pentole, olle, coperchi) ma a queste due categorie principali si possono integrare anche le mortaria, i grandi vasi usati anche come mezzi agricoli, e il vasellame votivo. Benché l'individuazione dei piccoli frammenti sia difficile, abbiamo tentato di distinguere le suppellettili fittili da mensa dalla ceramica da fuoco ed entro l'ambito di quest'ultima tipologia abbiamo cercato di individuare il vasellame o contenitori e il pentolame da fuoco. Abbiamo ricevuto un sostegno di rilievo dalla monografia di G. Olcese, dall'analisi riassuntiva del materiale dei luoghi di produzione dell'area Romana. $1 .^{18}$

\section{I REPERTI VENUTI ALLA LUCE DAL POZZO DELLA VILLA PIÙ ANTICA.}

Nel 1987 nella parte meridionale della chiesa medievale costruita trasformando il sudatorium sito nel lato orientale della villa abbiamo trovato un muro della chiesa, orientato in direzione $\mathrm{E}-\mathrm{O}$, la cui parte superiore sfiorava quasi la superficie del suolo. Il muro costruito di pietre poste irregolarmente e di mattoni romani riutilizzati era stato edificato su una base composta di pietrame e poca malta e si collegava alla sporgenza settentrionale, quadrata, a muri doppi, pur essendone separata da uno spazio di $10 \mathrm{~cm}$. Verso Est si osservava un lieve cedimento causato probabilmente dalla cisterna di epoca romana (Fig. 2). A $52 \mathrm{~cm}$ dalla superficie, tra le pietre del muro, abbiamo trovato una moneta di argento del XIV sec. Del muro si è conservata una parte di 45 centimetri. Sotto il muro è venuto alla luce un largo strato di crollo composte nella parte occidentale principalmente di mattoni, mentre verso E di pietrame, ma nel corso degli scavi si sono rinvenuti anche grandi blocchi bugnati e la ripulitura del muro ha portato alla luce frammenti bianchi e rossi di dipinti murali, piastrelle di marmo, pezzi di vetro da finestra, nonché tasselli per mosaico di vetro blu. Nello strato di detriti oltre a questi reperti d'epoca romana sono venuti alla luce solo frammenti di ceramica medievale, più che altro frammenti di vasi di ceramica invetriata ${ }^{19}$. Nel taglio g-4 tracciato per ricostruire la pianta della chiesa abbiamo recuperato anche due tombe medievali (le tombe $\mathrm{N}^{\circ} 22$ e 23 ), orientate all'incirca nella direzione dell'asse della chiesa. A circa 70-90 cm dalla superficie aprendo un varco attraverso uno strato d'incendio abbiamo trovato resti carbonizzati sporadicamente; in questo strato sono venute alla luce ceramiche medievali invetriate, pentole con ansa piatta, a nastro, un coperchio e alcuni ossi animali, nonché un blocco abbastanza grande con 40 tasselli bianchi. Sotto lo strato d'incendio abbiamo potuto osservare un seguente strato di detriti contenente una grande quantità di ceramiche medievali. Tutti gli strati, e particolarmente lo strato d'incendio, indicavano una profonda cisterna sottostante, giacché questo strato allungatosi in profondità per un mezzo metro mostrava i contorni di una cavità. Si è toccato il fondo dello strato a $180 \mathrm{~cm}$ dalla superficie con sotto macerie di malta e poche pietre e a una profondità di $265-270 \mathrm{~cm}$ si trovarono le tracce di una cavità a forma di un cerchio quasi regolare questa era la fossa della cisterna, riempito di macerie contenenti pietre e mattoni. La parte superiore era composta di humus e poca calce o malta. A $280-300 \mathrm{~cm}$ di profondità continuavano a venire alla luce detriti di ceramiche medievali, in maggior parte anse di vasi invetriati, ma a 290-295 cm apparvero anche frammenti di vasi d'epoca romana, per esempio una terra sigillata tipo Consp. 34 e un pezzetto pertinente a una tazza di color grigio-nerastro, decorata alla barbottina, di ceramica a pareti sottili, frammenti di lucerne e anfore, mentre a una profondità di $297 \mathrm{~cm}$ si trovò una moneta di Domiziano, coniata nell' 86 d.C.(BMC II. n. 393), qui, però il numero dei frammenti rinvenuti di piastrelle di marmo o di pitture murali era minore.

${ }^{17} \mathrm{~V}$. in lingua inglese: A. Martin: Ostia-Overview of the pottery. Acta RCRF 38 (2003) 46.

${ }^{18}$ G. OlCESE: Ceramiche comuni a Roma, in area romana. Produzione, circolazione e analisi di laboratorio. Doc. di Arch. 28. Mantova 2003.
19 Á. Kolláth: Le ceramiche medievali di San Potito. ActaArchHung. 62 (2011) 141, Cat.14, 16-17, 142 Cat. 22, 25, 143, Cat. 26, 145 Cat. 36. 

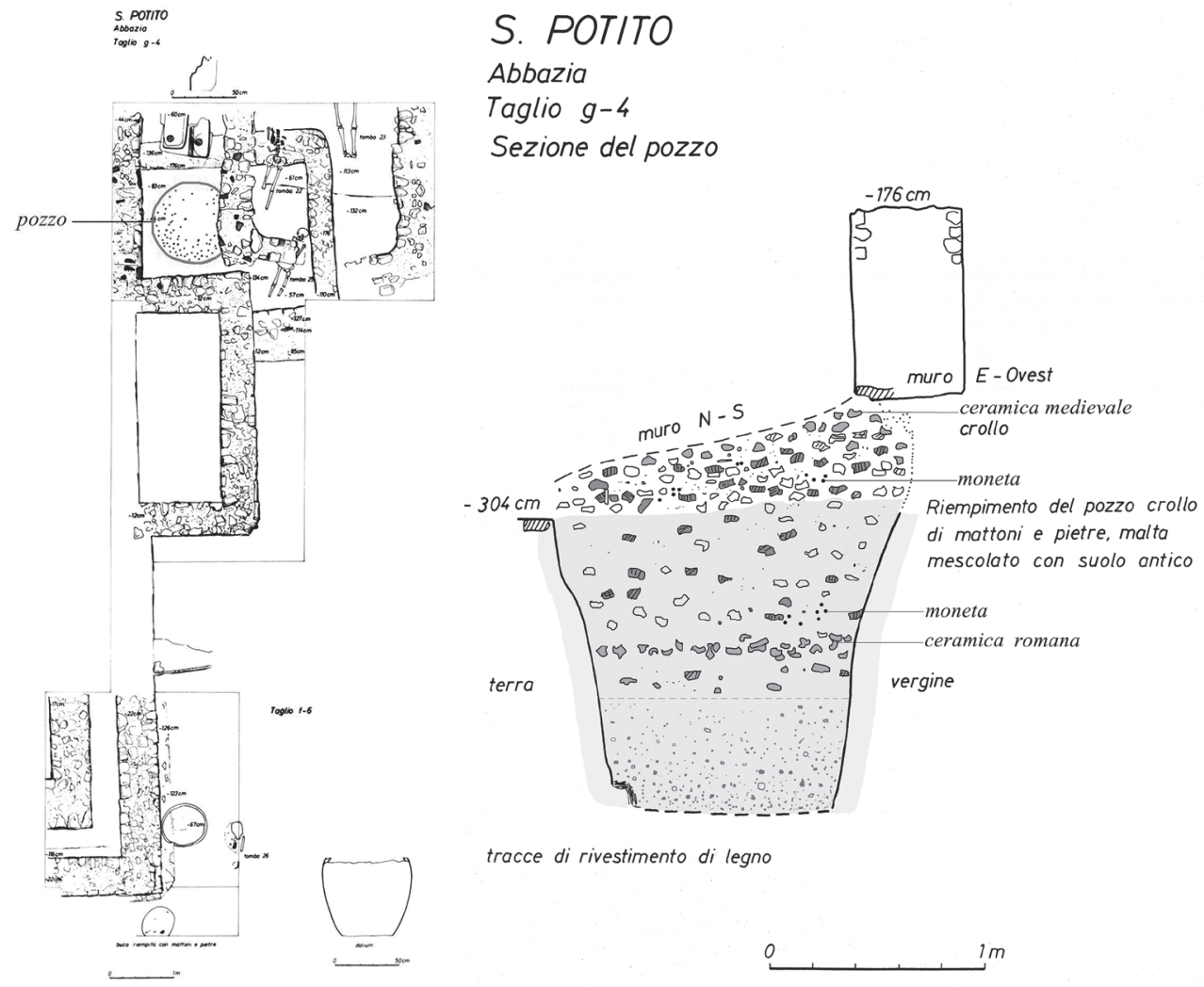

Fig. 2. Pozzo del taglio g-4 e la sezione del riempimento

Il pozzo fu scoperta tra la sporgenza settentrionale della costruzione a muri doppi e un muro orientato verso $\mathrm{N}-\mathrm{S}$ e nel corso della demolizione si osservarono degli strati giallognoli mescolati a malta e anche mattoni, nonché pietre di dimensioni piuttosto grandi. La distanza tra gli strati era in parte regolare. Il muro di orientazione N-S fu costruito sopra questo riempimento, di conseguenza la parte sottostante alle fondamenta verso Nord è più alta di circa $40 \mathrm{~cm}$ del resto, rivelando un cedimento del muro provocato dal pozzo. Il muro summenzionato, orientato da E verso O, è stato posto su questa struttura, mentre nel Medioevo il morto della tomba $\mathrm{N}^{\circ} 22$ fu sistemato su questo muro tolto. Nella parte meridionale del muro, sotto lo strato con mattoni, a 280-305 cm dalla superficie, vennero trovate macerie composte di pietre, carbonella, mattoni in una miscela maltosa. Procedendo all'ingiù la cavità della cisterna diventava sempre più stretta, il diametro che all'inizio misurava $170 \mathrm{~cm}$ in fondo era meno di 1 metro, allargandosi nella parte settentrionale. Ambedue i muri, quello orientato da $\mathrm{N}$ verso $\mathrm{S}$ e l'altro, orientato da $\mathrm{E}$ verso $\mathrm{O}$ sono stati costruiti su questa cavità riempita di detriti. Abbiamo portato avanti la demolizione fino a una profondità di $452 \mathrm{~cm}$. Nel riempimento si vedono diverse tracce lasciate dal livello della falda acquifera. Arrivando a 360-370 m abbiamo potuto portare alla luce una quantità relativamente maggiore di ceramiche d'epoca romana, trovando anche due monete di bronzo, quella più recente è un asse d'epoca traiana coniato nel 101/102 d.C., (BMC n. 751-752) e la moneta anteriore, un asse di Galba, coniato nel 69 d.C.(definito da F. Redö), fu ritrovato a $365 \mathrm{~m}$ di profondità, in cui si trovarono frammenti di terre sigillate, vasellame rosso con decorazione barbottina, anfore e ampolle, pentole, brocche. Oltre a queste vennero alla luce anche un listello di marmo, l'ansa a nastro di un vaso di vetro, tasselli per mosaico di impasto di vetro blu e due pezzi di piombo. Procedendo verso il fondo del pozzo, nel riempimento di ghiaia e pietrisco i reperti divennero più rari. A $120 \mathrm{~cm}$ dal livello in cui si è potuto avvistare il pozzo, abbiamo notato una colorazione scura che, copriva grosso modo tutta la circonferenza della cavità. Si ipotizza che questa colorazione più scura e meno compatta dell'orlo del buco possa essere la traccia o il resto della struttura di legno del pozzo. Il pozzo fu scavata in terra vergine e non ci fu possibile arrivare fino in fondo perché in questa profondità i lavori presentavano troppi rischi. 


\section{II.a Ceramica a pareti sottili}

Nell'ormai lontano 1986 sono stati pubblicati frammenti di questa tipologia fittile, provenienti dal materiale della villa di San Potito $^{20}$. Una coppa biansata, decorata a rotella $1^{21}$, due coppe con decorazione alla barbottina ${ }^{22}$ e una coppetta liscia ${ }^{23}$. Tutti i pezzi sono di color beige tendente al rosso e la superficie è coperta di vernice rosso-giallastra. Nessun pezzo può essere valutato dal punto di vista stratigrafico, essendo venuti alla luce da humus arato, da uno strato di frantumi di pietre o dalla cavità formatasi durante la demolizione del muro eseguita nella parte meridionale del cortile centrale.

L'importanza di questo tipo di ceramica ci ha indotti a pubblicare tutti i pezzi rinvenuti durante i lavori di scavo e non solo il materiale dei tre contesti chiusi di reperti.

Abbiamo trovato in tutto 40 pezzi:

taglio g-4, pozzo

cortile settentrionale, cisterna

strato d'incendio dell'edificio sito nella parte $S$ della villa più antica

altri siti

$\begin{array}{cccc}\text { Orlo } & \text { Parete } & \text { Profilo intero } & \text { Totale } \\ 5 & 7 & & 12 \\ 7 & 2 & 1 & 10 \\ 3 & 2 & & 5 \\ 5 & 5 & 3 & 13\end{array}$

L'impasto del vasellame a pareti sottili generalmente è di color marrone rossastro, beige rossastro, ocra pallido, l'argilla è depurata a dovere, farinosa. La superficie della maggior parte è verniciata. Solo in 3 casi l'argilla ben decantata è grigia ( $\mathrm{g}$-4 è lo strato d'incendio della villa più antica), in alcuni casi la superficie è grigio-nerastra. Solo un vaso mostra ingobbio.

La decorazione alla barbottina compare su 7 pezzi:

$\begin{array}{ll}\text { g-4 pozzo } & 1 \\ \text { il cortile settentrionale, cisterna } & 1 \\ \text { lo strato d'incendio della villa più antica } & 2 \\ \text { altri siti } & 3\end{array}$

La decorazione può essere costituita da foglie d'acqua (taglio g-4, parte S della villa, strato d'incendio) ed in un caso abbiamo trovato foglie a spina (cortile settentrionale, cisterna).

In 4 casi abbiamo trovato sui vasi una decorazione a rotelle.

$\begin{array}{ll}\text { il cortile settentrionale } & 1 \\ \text { lo strato d'incendio della villa più antica } & 1 \\ \text { altri siti, stratigraficamente non valutabili } & 2\end{array}$

Questa tipologia fondamentalmente fa parte della cosiddetta ceramica comune, al massimo può essere ritenuta un sottogruppo, non tanto in base al metodo di fabbricazione quanto sulla base della funzione ${ }^{24}$. Questo vasellame da mensa comprende vasi potori e contenitori di liquidi e vasellame per servire bevande, principalmente bicchieri e tazze, in certi casi la merce fittile sostituisce il vasellame più costoso di vetro o metallo. La ceramica a pareti sottili appare in varie forme o variazioni, se ne può osservare la fabbricazione nel periodo che va dal II sec. a. C. fino al III sec. d. C. ${ }^{25}$ Pur essendo le varie tipologie esportate nelle pro-

${ }^{20}$ D. GABLER-F. REDÖ: Gli scavi nella villa romana a San Potito di Ovindoli (L'Aquila) 1983-1984. ActaArchHung 38 (1986) 74-75.

${ }^{21}$ IBIDEM Fig. 34,2.

${ }^{22}$ IBIDEM Fig. 34, 3-4.

${ }^{23}$ IBIDEM Fig. 34,5.

${ }^{24}$ E. SChindleR-KaudelKa: Die dünnwandige Gebrauchskeramik von Magdalensberg 2. Die pareti sottili vom Südhang des
Magdalensberg. Die Ausgrabungen auf dem Magdalensberg 1980 bis 1986. Klagenfurt 1998, 390-391.

${ }^{25}$ M. VEGAS: Difusión de algunas formas de vasitos de paredes finas. Acta RCRF 5-6 (1963-1964) 61-83 L. Gervasini: La ceramica a pareti sottili, in: D. GANDOLFI: La ceramica e i materiali di età romana. Classi, produzioni, commerci e consumi. Bordighera 2005, 279. 
vince $^{26}$ ben conosciute dai ricercatori, tuttavia fu N. Lamboglia a scoprirne l'importanza ${ }^{27}$. Le sue ricerche acquistarono maggior slancio all'inizio degli anni Settanta con la pubblicazione del materiale di Sutri ${ }^{28}$, uno dei luoghi di produzione nonché con la comunicazione dei siti di maggior rilievo, fra cui sono da evidenziare $\mathrm{Cosa}^{29}{ }^{2}$ Luni $^{30}$, Ostia $^{31}$, Russi, Porto Recanati, Angera ${ }^{32}$, Emona $^{33}$. Le analisi tipologiche e stratigrafiche e la seriazione cronologica di particolare rilievo dal punto di vista dei luoghi di fabbricazione dell'Etruria di M. T. Marabini rivestono per noi una straordinaria importanza. Nel corso della pubblicazione del materiale della Casa delle Pareti Gialle di Ostia I. Pohl e F. Zevi hanno potuto localizzare un altro centro di produzione dell'Italia Centrale i cui motivi decorativi ricorrono su varie forme di vasellame. La decentralizzazione della produzione ebbe come conseguenza la nascita di una grande varietà degli impasti, dei colori e dei motivi decorativi adottati dalle diverse officine per la produzione della medesima tipologia, e anche la capienza dei vasi permette solo una standardizzazione regionale. È ipotizzabile inoltre, che anche le usanze culinarie delle singole regioni potessero contribuire alla diversità dell' assortimento di forme adottate nei singoli siti. Tra i reperti ritrovati nelle province il materiale di Magdalensberg con la sua facile datazione ${ }^{34} \mathrm{e}$ i prodotti venuti alla luce in Baetica ${ }^{35}$ rivestono un interesse straordinario. A. Ricci ha pubblicato uno studio minuzioso, riguardante le ceramiche a pareti sottili avendo analizzato il materiale non solo dal punto di vista morfologico, ma tentando anche di separare i luoghi di produzione e i relativi mercati, definendo in tal modo l'importanza commerciale di questa merce. La sua attività con l'introduzione del concetto della ceramica di tradizione a pareti sottili aggiunge alle ricerche i tipi di vasi che, indipendentemente dallo spessore delle pareti, possono essere inquadrati in questa tipologia, benché questi tipi di epoca tarda possano essere attribuiti alla categoria di ceramica comune ${ }^{36}$. Questi fanno parte anche dei contesti da noi rinvenuti.

Le forme della ceramica a pareti sottili sono molto varie, però fondamentalmente possono essere attribuite a due tipi primari, alle coppe e alle tazze. Tra le decorazioni vale la pena di menzionare la sabbiatura, l'incisione a pettine, nonché la decorazione a rotella o alla barbottina spesso adottati anche a San Potito ${ }^{37}$. Nel nostro materiale, compaiono i motivi del tipo barbottina che si definiscono "alle foglie d'acqua" e la decorazione a spine.

Si ipotizza che le prime officine in cui si fabbricarono ceramiche a pareti sottili fossero attive nella regione settentrionale del Lazio e nell'Etruria del S. All'inizio i vasi probabilmente giunsero nei diversi punti del Mediterraneo come merce commerciale ${ }^{38}$, in epoca augustea poi vennero costituite nuove officine ad Aquileia, nella Valle Padana e a Siracusa. Abbiamo poche notizie riguardanti i luoghi di produzione e di conseguenza le informazioni deducibili dalla merce a pareti sottili e riguardanti i rapporti commerciali, possono essere prese in considerazione solo limitatamente. Non si può stabilire con certezza se nelle singole officine fosse fabbricata proprio questa tipologia fittile. Generalmente furono prodotte solo coppe e tazze e in questo periodo ha inizio la verniciatura dei vasi. Nel I sec. anche nelle province, quindi anche in Pannonia, sorsero le officine in cui si fabbricavano questi tipi di vasi. Oltre alle officine summenzionate della Baetica la produzione gallica era pure notevole. Le ceramiche a pareti

${ }^{26}$ CHR. SimonetT: Tessiner Gräberfelder. Basel 1941-passim; É. Bónis: Die kaiserzeitliche Keramik von Pannonien I. DissPann ser.II. 20. Budapest 1942, 27-30.

${ }^{27}$ N. LAmBogLiA: Gli scavi di Albintimilium e la cronologia della ceramica romana I. Campagne di scavo 1938-1940. Bordighera 1950; Idem: La nave romana di Albenga. RivStLiguri XVIII.3-4 (1952) 17-175, 182.

${ }^{28}$ G.C. DunCAN: A Roman Pottery near Sutri. PBSR 32. n.s. 19 (1964) 38-88.

${ }^{29}$ M.T. Marabini MoEvs: The Roman thin walled pottery from Cosa (1948-1954) Roma 1973.

${ }^{30}$ AAVV: Scavi di Luni I. Relazione preliminare delle campagne di scavo 1970-1971 (a cura di A. Frova). Roma 1973; AAVV: Scavi di Luni II. Relazione preliminare delle campagne di scavo 1972-1974. (a cura di A. Frova) Roma 1977.

${ }^{31}$ I. PoHL-F. Zevi: Ostia. Casa delle Pareti Gialle. Salone Centrale. Scavo sotto il pavimento a mosaico. NotSc 24 (1970) suppl. 43-234; AAVV: Ostia I. Studi miscellanei 13 (a cura di A. Carandini) Roma 1968; AAVV: Ostia II. Studi miscellanei 16 (a cura di A. Caran- dini). Roma 1970; AAVV: Ostia III. Studi miscellanei 21 (a cura di A. Carandini). Roma 1973.

${ }^{32}$ AAVV: Angera romana. Scavi nella necropoli 1970 1979 (a cura di G. Sena Chiesa). Roma 1985, L. PleniCAR-GEC: The northern necropolis of Emona. Ljubljana 1972; P. Petru: Emonske nekropole. Ljubljana 1972.

${ }^{33}$ L. Plenicar-Gec: The northern necropolis of Emona. Ljubljana 1972; P. Petru: Emonske nekropole. Ljubljana 1972.

${ }^{34}$ E. SCHINDLER KaudelKa: Die dünnwandige Gebrauchskeramik vom Magdalensberg 2. Die pareti sottili vom Südhang des Magdalensberges in: Die Ausgrabungen auf dem Magdalensberg 1980 bis 1986. Klagenfurt 1998, 389-427.

${ }^{35}$ M. VEGAS: Cerámica común romana del Mediterráneo Occidental. Barcelona 1973; F. MAYET: Les céramiques à parois fines dans la Péninsule Ibérique. Paris 1975.

${ }^{36}$ A. RICCI: Ceramica a pareti sottili. Atlante delle forme ceramiche. Ceramica fine romana nel bacino del Mediterraneo (Tardo ellenismo e primo impero) II. Roma 1985.

${ }^{37}$ RiCCI 1985, tav. CI-CXIV.

${ }^{38}$ Gervasini 2005, tav. 3, 1-2. 
sottili videro il periodo di maggior fioritura nell'epoca flavia, dopodiché si osserva un lento declino della loro produzione. Nello stesso tempo emerge a Ventimiglia, Ostia e a Lipari il cosiddetto boccalino a collarino. Questa forma tarda fu fabbricata per tutto il II sec. (Marabini forma LXVIII), anzi, è ritrovabile perfino in un insieme di reperti del III sec. rinvenuto nell'agorà di Atene. Questi vasi che, dal punto di vista tipologico fanno quasi corpo con i prodotti di ceramica comune, è l'unica tipologia che rimase in circolazione nel II sec. ed all'inizio del III sec. Si ipotizza in base alla stratigrafia di Ostia, che, l'altro tipo di merce d'epoca tarda, la forma Mayet XXIV, fu prodotta fino all'epoca di Adriano ${ }^{39}$. A. Ricci sostiene che le caratteristiche qualitative e l'impasto dei tipi ritrovabili nei materiali di Roma e Ostia, indicano che furono prodotti sul luogo, pur non essendo state finora scavate le relative officine. Sulla base di queste caratteristiche si distinguono nettamente dai prodotti di Sutri, dell'Etruria o della Campania. Sono caratterizzati dall'argilla ben depurata e omogenea, poco cotta e non troppo dura, dallo spessore delle pareti dei vasi che è di 2-3 mm. L'argilla è color viola rossastro o beige rossastro, il rivestimento è di un rosso vivace tendente al viola. I pezzi decorati hanno per lo più una decorazione a rotella e raramente alla barbottina. La coppa probabilmente sparisce dal repertorio di fabbricazione dell'officina alla fine del I sec., mentre il boccalino monoansato rimane uno dei tipi principali di questo centro di produzione fino alla metà del II sec. Probabilmente, le officine centro italiche hanno iniziato la loro attività alla fine dell'epoca repubblicana, o al più tardi nell'epoca augustea ${ }^{40}$, che, suppergiù coincide con la crisi delle esportazioni di vasi di produzione etrusca. Le officine campane e siciliane non tardarono ad adottare le innovazioni etrusche. Comunque, la distinzione dei prodotti centro italici e etruschi richiede una massima circospezione, particolarmente se si tratta dei cosiddetti prodotti campani e centro italici. Le esportazioni dei prodotti provenienti dal/i luogo (luoghi) di produzione centro italico/i ebbero ampia diffusione, la protrazione di tale attività produttiva è attestata fino alla metà del II sec., ma, in base al complesso di reperti ritrovato a Scoppieto il cui 73\%-a è databile al periodo tra la fine del I e l'inizio del II secolo ${ }^{41}$ il periodo di maggior fioritura è databile alla seconda metà del sec. I e (Sutri, a Roma La Celsa e Prima Porta). La concorrenza delle officine provinciali, come quella di Baetica, però troncò quest'attività. La produzione delle officine della Renania e della regione danubiana ${ }^{42}$ guadagnò invece importanza proprio in quest'epoca, alla metà del II sec. Più tardi, nel mercato dell'area mediterranea rimase solo il boccalino 1/122 di Ricci probabilmente fabbricato nell'Italia Centrale o Meridionale ${ }^{43}$. Per quel che riguarda gli esemplari di argilla grigia, cotti in atmosfera riducente, nel nostro contesto figurano 3 frammenti di questo tipo che, oltre al litorale adriatico vennero alla luce anche in altri siti abruzzesi. Non è escluso, però che questi siano stati importati da Ravenna o dalle Marche. ${ }^{44}$ Nell'officina di Scoppieto fu prodotta anche una piccola quantità di ceramica grigia, circa il $4 \%$ dell'intera quantità di produzione ${ }^{45}$.

Nel nostro caso è difficile individuare l'officina, giacché i siti che dispongono di materiale comparativo, come Cosa, Bolsena o, Benevento, Ordona (Herdonia), a Roma La Celsa, Sutri o anche Scoppieto ${ }^{46}$ sono situati molto lontano dal territorio abruzzese. (In base a fonti scritte pare che, le caratteristiche dei materiali e dei rivestimenti adottati dall'officina di Benevento siano simili, ma ciò non permette però di collegare decisamente le tipologie a parete fine a questo luogo di produzione).$^{47} \mathrm{E}$ ciò è ancor maggiormente vero in riferimento ai luoghi di produzione padani. Molte volte, nel caso di officine "attestate" solo dall'uso dei colori o dalle forme, o dalle fornaci o altri mezzi adottati per la produzione, non si può stabilire se si tratti di figline di rilievo locale o officine che producevano anche per esportazione, al massimo è la merce italica ritrovata nelle province lontane, in cui per un lungo periodo non si sviluppò la produzione locale che, indica l'esistenza di esportazioni ad ampio raggio che coinvolsero grandi territori.

Sono pochi i reperti venuti alla luce a San Potito, di cui si poté definire più precisamente la forma, infatti, in molti casi si sono conservati solo piccoli frammenti della parete. La coppa emisferica, di forma Mayet XXXV, con rivestimento marrone $\mathrm{o}$ arancione rossiccio fu prodotta già dall'epoca di Tiberio. La produzione della coppetta

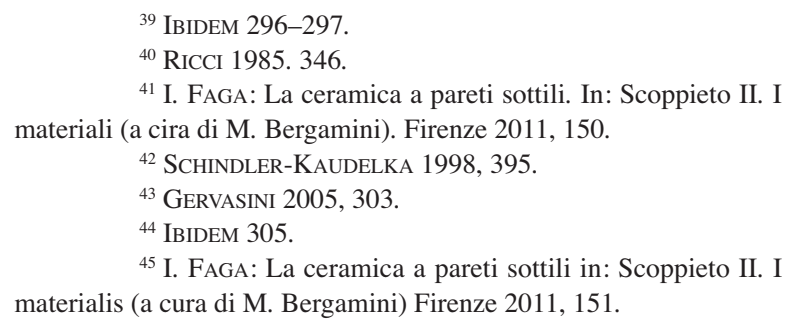

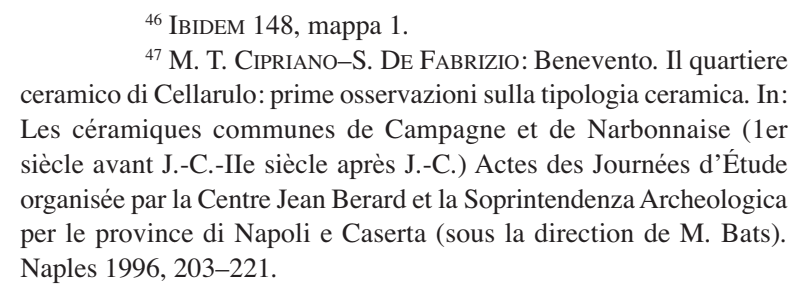

${ }^{47}$ M. T. Cipriano-S. De Fabrizio: Benevento. Il quartiere ceramico di Cellarulo: prime osservazioni sulla tipologia ceramica. In: Les céramiques communes de Campagne et de Narbonnaise (1er siècle avant J.-C.-IIe siècle après J.-C.) Actes des Journées d'Étude organisée par la Centre Jean Berard et la Soprintendenza Archeologica per le province di Napoli e Caserta (sous la direction de M. Bats). Naples 1996, 203-221. 
di forma Mayet XXX ebbe inizio circa nella stessa epoca. ${ }^{48} \mathrm{Al}$ terzo quarto del I sec. d. C. possono essere datate le coppe ansate globulari. ${ }^{49} \mathrm{Il}$ periodo di diffusione delle varianti color ocra o tendente al rosa della coppa Mayet XXXVII, con decorazioni a rotella o alla barbottina durò dall'epoca tiberiana fino all'inizio del II sec.; oltre ai prodotti ben cotti e duri si conoscono anche pezzi sgretolati e farinosi ${ }^{50}$. Si ritiene che, la coppa di forma Mayet XLIII decorata a rotella, prodotta di materiale color ocra e con rivestimento rosso-arancione sia di origine iberica. Secondo L. Gervasini questa tipologia si è diffusa in territorio iberico, però alcuni esemplari sono noti anche come provenienti da $\operatorname{Cosa}^{51}$. Il nostro esemplare probabilmente deriva da un'officina centro italica, benché nel nostro materiale la proporzione delle anfore di provenienza ispanica sia rilevante ${ }^{52}$. La coppetta rientra nel tipo Ricci 2/222 ed è vicina ad esemplari provenienti dalla fornace La Celsa, datati della prima metà del I sec al $70 \mathrm{~d}$. C. ${ }^{53}$ Una delle forme più diffuse è il boccalino di tipo Mayet XXXIV. Le importazioni dell'Italia Centrale, provenienti da Baetica, databili alla seconda metà del I sec. d. C. sono insignificanti. ${ }^{54}$

\section{Catalogo delle ceramiche a pareti sottili.}

1. Fra le ali costruite attorno al cortile centrale e meridionale, nel taglio 2, a 60-65 cm di profondità, in uno strato dal suolo pietroso e calcinoso del taglio 1, è venuta alla luce una coppetta biansata con orlo verticale e profilo arrotondato, simile alla Marabini LXIII. L'ansa con due solcature è impostata sulla spalla. Su diverse fasce del corpo si vedono decorazioni a rotella ${ }^{55}$. Argilla ocra-bruna, vernice bruno-nerastra, lucente e metallica.

Seconda metà del I sec. d. $\mathrm{C}^{56}$

2. Nella parte E della sezione 7, dall'humus arato è stato scavato un fr. di parete di una tazza decorata alla barbottina con una foglia d'acqua e tralci. Argilla depurata di colore rosso-ocra. La parete esterna è rivestita di vernice bruna. ${ }^{57}$ Non si è potuto definire la forma a causa della frammentarietà dell'esemplare. ${ }^{58}$

3. Angolo SE del cortile centrale, crollo (12/10/1996). Fr. Di una tazza decorata alla barbottina con luna crescente e puntini a ri$\operatorname{lievo}^{59}$ v. Marabini Moevs 1973, 83, tipo 2560. (Fig. 3.1) Epoca flavia.

4. Il boccalino biansato, venuto alla luce nel 1998 (5/11) nell'ambiente ubicato a O. dall'entrata principale del secondo periodo della villa, può essere inquadrato forse tra i reperti d'epoca tarda. Boccalino biansato a pareti sottili (2,5-3 mm) ricomposto da 14 frammenti. La parte inferiore del corpo è arrotondata, la parte superiore troncoconica. Residuo di un frammento di orlo. Due anse a nastro con due solcature profonde, impostate tra collo e spalla. Fondo ad anello. Labbro ricurvo verso l'esterno. Sulla spalla decorazione a rotella. Presenta corpo ceramico depurato, giallo arancione, un po' farinoso, rivestito di vernice rossa-marrone. (Quest'ultima si è conservata solo in tracce). (Fig. 3.2, Fig. 25.1) La forma mostra le caratteristiche formali dei tipi Marabini LXXI (Ricci /45) e Marabini LXXV(=Ricci 1/82) ${ }^{61}$. Periodo di produzione ipotizzabile dall'epoca claudia ${ }^{62}$ fino all'inizio del II sec. ${ }^{63}$

${ }^{48}$ Schindler-KaudelKa 1975, forma 134; E. Schindler-Kaudelka: La ceramica a pareti sottili del Magdalensberg 19751998-2011, in: Emona between Aquileia and Pannonia. (ed. I. Lazar-B. Županek) Koper 2012, fig. 14,23.

${ }^{49}$ FAGA 2011, 139.

${ }^{50}$ IBIDEM 297.

${ }^{51}$ IBIDEM 297.

${ }^{52}$ P. HÁRshegyi: Le anfore della villa romana di San Potito, in: D. Gabler-F. Redő: Ricerche archeologiche a San Potito di Ovindoli e le aree limitrofe nell' antichità L'Aquila 2008, 140, 164.

${ }^{53}$ M. CARrara: Prima porta. La collina della torre. Bollettino della Commissione Archeologia Comunale di Roma 99 (1998) 389.

${ }^{54}$ FAGA 2011, 147.

${ }^{55}$ DunCAN 1964, 33; M. VeGas: Cerámica común romana del Mediterráneo Occidental. Publ. eventuales 22. Univ. de Barcelona 1973, Fig.27, tipo 34,5.

${ }^{56}$ Pubblicato da: D. GABLER-F. REDÕ: Gli scavi nella villa romana a San Potito di Ovindoli (L'Aquila) 1983-1984. ActaArchHung 38 (1986) 74, Nr. 14 Fig 34, 2.
${ }^{57}$ M.T. Marabini-Moevs: The thin walled pottery from Cosa (1948-1955) MAAR 32 (1973) pl. XLV; M. Bergamini: La ceramica romana. Quaderni degli Studi Romagnoli 8. Faenza 1973, 57; L. Mazzeo Saracino: Russi (Ravenna). Campagne di scavo 1971. NotSc 31 (1977) 5 ss.; M.P. Lavizzari-Pedrazzini: Angera romana. Scavi nella necropoli 1970-1979. (a cura di G. Sena Chiesa) Roma 1985, 406.

${ }^{58}$ Pubblicato da: GABLER-REdō 1986, 74, Nr.15, fig. 34,3.

${ }^{59}$ DunCAN 1964, fig. 8,42.

${ }^{60}$ E. SCHINDLER-KAUDELKA: La ceramica a pareti sottili del Magdalensberg 1975-1998-2011. In: Emona between Aquileia and Pannonia. (ed. I. Lazar-B. Županek) Koper 2012, fig. 14,7.

${ }^{61} \mathrm{Ld}$. Settefinestre. Una villa schiavistica nell' Etruria romana (a cura di A. Carandini-A. Ricci). Modena 1985, tav. 45,4.

${ }^{62}$ M.T. Marabini-MoEvs: The thin walled pottery from Cosa (1948-1955) MAAR 32 (1973) 257.

${ }^{63}$ S. MASSERoli: Ceramica a pareti sottili in: Calvatone romana. Un cisterna e il suo contesto. (A cura di G. Sena Chiesa-S. Masseroli-T. Medici-M. Volontè) Quaderni di ACME 29, 70 . 

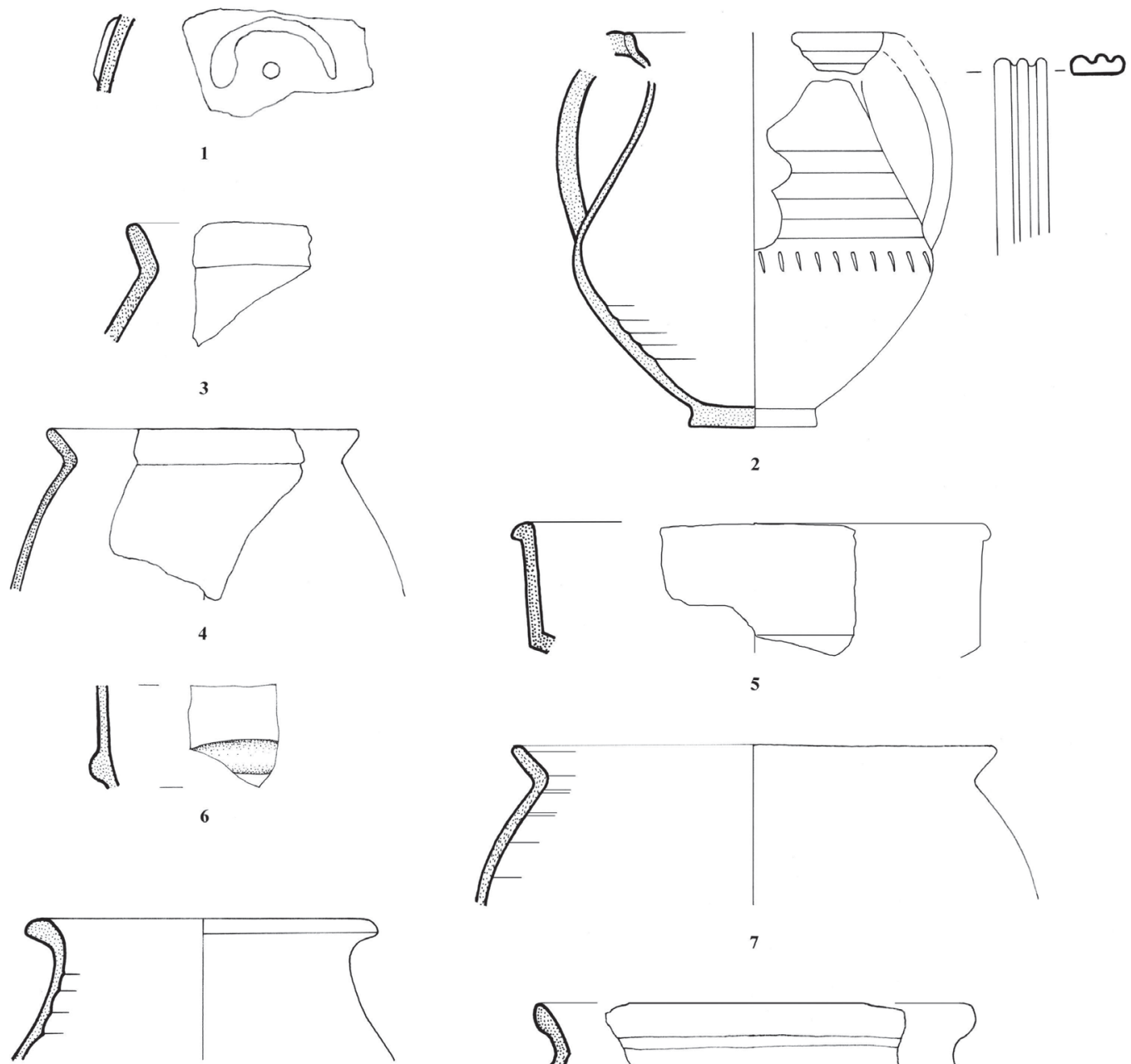

7

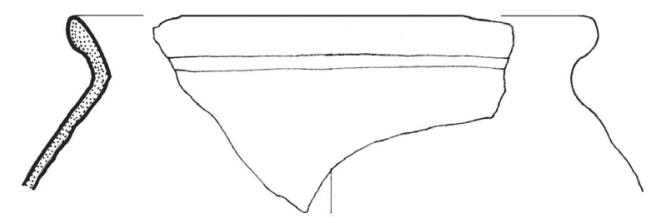

9

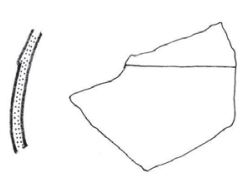

10
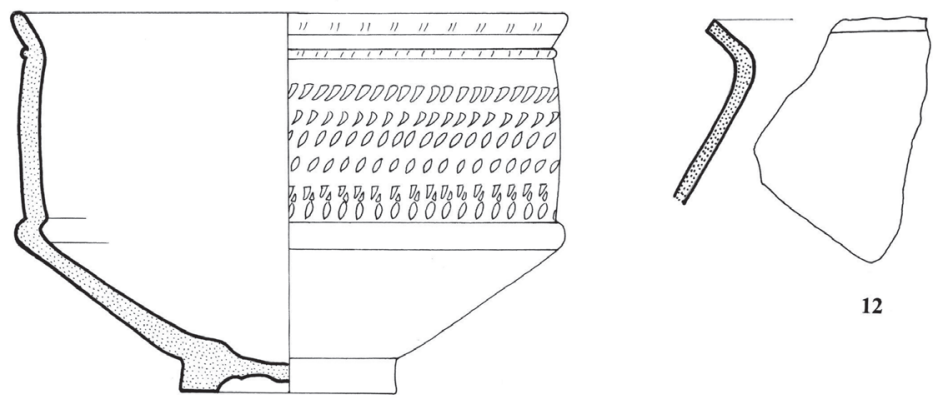

12

11

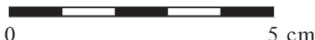

Fig. 3. Ceramica a pareti sottili dal pozzo del taglio g-4. 
5. Nella cavità del taglio 8 , ad una profondità di 35-60 cm è venuta alla luce una coppetta a corpo emisferico, con labbro sottolineato da una solcatura orizzontale Marabini XXXVI=Ricci 2/407. Argilla beige tendente al rosso, vernice arancio-bruna, opaca. ${ }^{64}$ Probabilmente è del I sec. ${ }^{65}$

6. Nel riempimento del pozzo scavato nel taglio $\mathbf{g}-4$ a $350-370 \mathrm{~cm}$ di profondità abbiamo ritrovato un fr. di un boccalino con collarino. Argilla ben depurata, giallo-arancione. (Fig. 3.3) Seconda metà del I sec.

7. Fr. di un boccalino a corpo globulare con collarino Ricci tav.XXXIV, tipo 14, forma 1/111. Argilla ben depurata, rosso arancione, digrassata con alcuni grani di sabbia ${ }^{66}$. (Fig. 3.4) Seconda metà del I sec.

8. Fr. di una coppa (biansata?) a carena molto bassa e spigolo vivo. Le pareti sopra della carena, sono verticali. L'orlo è di piccole dimensioni, diritto e inclinato verso l'esterno. V. Mayet XXVII= Marabini XL= Ricci 2/249. Argilla piuttosto fine, di colore giallo-arancione. (Fig. 3.5)

9. Fr. di una coppa dello stesso tipo. Argilla giallo-arancione.

10. Fr. di una coppa dello stesso tipo. Argilla dura, ocra.

11. Fr. di una coppetta carenata a parete verticale. Sulla parete foglia alla barbottina. Argilla gialla ocra, vernice: marrone consumata. ${ }^{67}$. Fig. 3.6)

12. Fr. di un bicchiere a pareti con sezione arrotondata. Il labbro ingrossato è inclinato verso l'esterno. v. Marabini LXX; Duncan fig. 7,7. Argilla giallo-arancione, con particelle micacee di colore bianco. ${ }^{68}$

13. Fr. di un bicchiere a pareti con sezione arrotondata. Il labbro ingrossato è inclinato verso l'esterno, forma Sutri 1 (Ricci 1/123), Duncan 1964 fig.7,7. Dm:10,8 cm. Argilla giallo-arancione con particelle micacee di colore bianco. (Fig. 3.7)

14. Fr. di parete di un bicchiere con parete arrotondata. Il labbro è inclinato verso l'esterno tipo Ricci 1/102, tac. LXXXIV tipo $8=$ Mayet XXIV. Dm:8 cm. Argilla giallo-arancione, digrassata con alcuni grani di sabbia (Fig. 3.8)

15. Fr. di parete di un bicchiere. Argilla molto fine di colore ocra. (Fig. 3.10)

16. Fr. della parete di un di un boccalino a parete arrotondata. Argilla dura, grigia, vernice rossa. V. Duncan 1964, forma 1 Fig. 7.9.

17. Fr. di orlo e parete di un vaso a parete globulare. Argilla giallo-ocra, vernice marrone.

18. Fr. della parete di un vaso a parete globulare. Tracce di tornitura. Argilla giallo arancione, la parete interna è marrone.

19. Fr. di orlo e parete di un vaso con collarino, v. Duncan 1964, forma 1 (Ricci 1/123). Argilla giallo ocra (Fig. 3.9)

20. Taglio d+11, saggio fuori i muri, accanto alla buca per il canale, sotto il livello del basamento del muro N-S, crollo di mattoni (3/10/1995). Fr. di un boccalino a corpo globulare con collarino, v. Marabini XLVI ${ }^{69}$. Argilla depurata, giallo-arancione, vernice: rosso-marrone. (Fig. 3.12) (gruppi II)

Fine del I sec. d. C

21. Nel riempimento del pozzo scavato nella parte centrale del cortile settentrionale, nel taglio t+4 il 2 ott. 2001 è venuta alla luce una coppa carenata ad orlo distinto, a parete verticale con curvatura a spigolo vivo. Sulla parete decorazione a rotella in sei fasce. La decorazione compare anche sull'orlo. La parete inferiore è troncoconica. Piede ad anello. La parete è alquanto spessa (4 mm). Altezza: 7,1 cm, dm (dell'orlo): 10,5 cm, dm (fondo): 4,2 cm Argilla depurata giallo-arancione, ingobbio rosso-marrone. (Fig. 3.11) La forma mostra le caratteristiche formali dei tipi Mayet XLIII= Ricci 2/248. Per la datazione si può tener conto del periodo dalla metà del I sec. ${ }^{70}$ fino al II sec ${ }^{71}$. È emersa l'ipotesi della produzione iberica di tale tipologia ${ }^{72}$.

22. Nel medesimo luogo sono stati trovati tre fr. di una coppetta ad orlo distinto, a parete verticale. La parte inferiore è arrotondata, con una solcatura sulla parete interna. ${ }^{73}$ Mayet XXX; Ricci 2/327; Luni I. Tav.0, 1 CM 532/8; Brecciaroli Taborelli 2010, fig. 116,12. Argilla depurata giallo-arancione. (Fig. 4.1.) Epoca flavio-adrianea.

23. Similmente, nel riempimento della cisterna situata nella parte centrale del cortile N, è venuto alla luce anche un vasetto di forma tendenzialmente emisferica, leggermente più allungata. Orlo leggermente estroflesso; nel corpo uno spigolo. Presenta un fondo

${ }^{64}$ DunCAN 1964, 30, forma 6.

${ }^{65}$ Publ. Gabler-Redó 1986, 74, Fog. 34,5.

${ }^{66}$ Duncan 1964, fig.7,12; A. Carbonara-G. Messineo: La Celsa (circ. XX). Bollettino della Commissione Archeologica di Roma 1991-1992, 243, 5.

${ }^{67}$ Carbonara-Messineo 1991-1992, 244, 7 b.

${ }^{68}$ IBIDEM p. 184, fig. 243, 1 b.

${ }^{69}$ Confronti puntuali a Napoli dal complesso archeologico di Carminiello ai Mannesi - v. I. Faga: Ceramica a pareti sottili dal complesso archeologico di S. Lorenzo MagGiore (Napoli). Acta RCRF 40 (2008) fig.6,2.

${ }^{70}$ L. BRECCIAROLI TABORELli: Ceramica a pareti sottili in: Oro, pane e scrittura. Memorie di una comunità "inter Vercellas et Eporediam' (a cura di L. Brecciaroli Taborelli) 2010, fig.116,13 b-c.

${ }^{71}$ I. FAGA: Ceramica "a pareti sottili" della prima età imperiale dal porto di Neapolis. Acta RCRF 41 (2010) fig. 5,4.

${ }^{72} \mathrm{~V}$. nota 25.

${ }^{73}$ Duncan 1964, p. 75, forma 6, Fig. 8,30. 
piano, con piccolo piede ad anello, inquadrabile nel tipo di forma A. Ricci 2/28174. (Simile a Mayet XXXVII). Argilla di colore beige rosato, il rivestimento è di colore rosso con mica. Sulla parete decorazione a rotella in 5 fascie (Fig. 4.2)

Non è possibile definire un centro di produzione.

Fine del II sec. d. $\mathrm{C}^{75}$

24. Fr. di un boccalino con collarino a pareti sottili Marabini XLVI. Argilla depurata, giallo-arancione. (Fig. 4.3) Fine del I sec.

25. Fr. di un boccalino a corpo globulare con collarino. Sulla parete decorazione a rotella in 4 fasce. v. Duncan 1964, forma 1, fig.

7,16. Argilla rosso-arancione. (Fig. 4.4)

Simile alla tipologia della ceramica a pareti sottili.

Prima metà e fine del II. sec. ${ }^{76}$

26. Fr. di un boccalino a corpo globulare con collarino. Sotto l'orlo un listello. v. Duncan 1964, forma 1, fig.7,20.(Fig. 4.5) Argilla ocra

27. Fr. di una coppa. Le pareti piuttosto rigonfie in prossimità dell'orlo si ristringono rapidamente verso il fondo. All'altezza dell'imboccatura le pareti si inclinano leggermente verso l'interno. v.. Ricci tipo 2/307. Argilla rossiccia, farinosa, vernice rossa, all'altezza dell'orlo marrone ${ }^{77}$ (Fig. 4.6)

Fine del I - inizio del II sec.

28. Coppa a parete leggermente arrotondata. Il labbro a sezione circolare; una solcatura rileva il labbro. Decorazione alla barbottina con una fascia di motivi a spina.(Ricci tav. CVII tipo 8) Ostia, suppl. XXX (1978) 1987, fig. 91,38. Confronto della forma:

Schindler-Kaudelka 2012, fig.15,23. Argilla dura, giallo-marrone ${ }^{78}$ (Fig. 4.7)

Seconda metà del I sec. d. C

29. Fr. di una coppa tendenzialmente emisferica. L'orlo estroverso, a sezione circolare è rilevato da una solcatura ${ }^{79}$. v. Ricci tav. XCII. 9; Marabini 1973, tav.24, n.231; Luni I. tav.60, 1 CM 532,8. Argilla rosso arancione, della vernice rossa si sono conservate solo alcune tracce (Fig. 4.8)

II sec.

30. Fr. di una coppa tendenzialmente emisferica. Sotto l'orlo due solcature. Mayet XXXIII.

Argilla: all'interno rivestimento nero grigiastro, all'esterno vernice marrone, tendente al grigio con inclusioni notevoli di calce. (Fig. 5.1)

31. Sul lato meridionale del "rafforzamento" rettangolare, tra i due muri, nello strato d'incendio è stato trovato (9/10/2008) un fr. di parete di una tazza. Argilla depurata, rosso arancione (Fig. 4.9)

32. Sul lato meridionale del "rafforzamento" rettangolare, tra i due muri, nello strato d'incendio è venuto alla luce un boccalino a parete arrotondata a forma Mayet XXIV=Ricci 1/102, simile alla forma Marabini LI, decorato alla barbottina con foglie d'acqua (Ricci 226). Argilla rosso -arancione, con inclusioni nere, internamente rivestimento rosso. Confronti: I. Faga: Ceramica a pareti sottili dal complesso archeologico S. Lorenzo Maggiore (Napoli). Acta RCRF 40 (2008) 401, fig.5,9., F. Fabbri: Some pottery productions from the kilns of Vingone in Scandicci (Florence, Italy). Acta RCRF 41 (2010) 183, fig.2,5. (Fig. 4.10) Dall'epoca augustea fino all'inizio del II sec.

33. Fr. di una coppa carenata. Le pareti sono quasi verticali con orlo diritto, inclinato verso l'esterno. Tipo Ricci 2/298, tav. XLI. 4. Il corpo è decorato a rotella. Vernice rossa ${ }^{80}$ (Fig. 5.2)

Seconda metà del I sec. d. C.

34. Dal medesimo sito è venuta alla luce una coppa a parete arrotondata, con orlo estroflesso. Argilla rosso-ocra, con inclusioni nere, v. Luni Tav.60, 4 CM 420,1; Luni II. Tav.245,24? I..; S. Menchelli O. Cerbone: Ceramiche fini nell'ager Firmanus (Fermo, Marche Meridionali). Fasti Online Documents and Research 2012. Tav.2,34 (Fig. 5.3)

Dalla fine del I sec. fino all'inizio del II.

${ }^{74}$ MAYET 1975, tav.XXVI, n. 198, p. 313.

${ }^{75}$ Brecciaroli Taborelli 2010, fig. 116, 17 b.

${ }^{76}$ H.PATTERSON-A. Bousquet-H.Di Giuseppe-F. FeliciS. FONTANA-R. WITCHER-S. ZAMPONI: Le produzioni ceramiche nella media valle del Tevere tra l'età repubblicana e tardoantica. Acta RCRF 38 ( 2003) fig.9,8.

${ }^{77}$ PH. M. KenRick: The fine pottery. Excavations at Sidi Khrebish, Benghazi (Berenice) III 1 Libya Antiqua, suppl.5 Tripoli 1985, 271-282.
${ }^{78}$ A. Carbonara-G. Messineo la Celsa (cic. XX). Bollettino della Commissione Archeologica di Roma 1991-1992, tipo 7 c.

${ }^{79}$ MAYET 1975, p.7, 78-79.

${ }^{80}$ La datazione per gli esemplari di Luni oscilla in base allo schema decorativo e fasce di rotellazione dall' età tiberiana a tutto il I. sec.d. C. Luni CAVAlieri-MANESSE 1975, Tav.60,10; M. CARTA-I. Pohl-F. Zevi: Ostia, La taberna dell'Invidioso. Piazzale delle corporazioni, portico ovest: saggi sotto i mosaici. Not Sc 8, ser.32 1978, suppl. Roma 1987 p. 110, fig.111,85; BRECCIAROLI-TABORELLI 2010, fig.116,9 - seconda metà del I sec. 

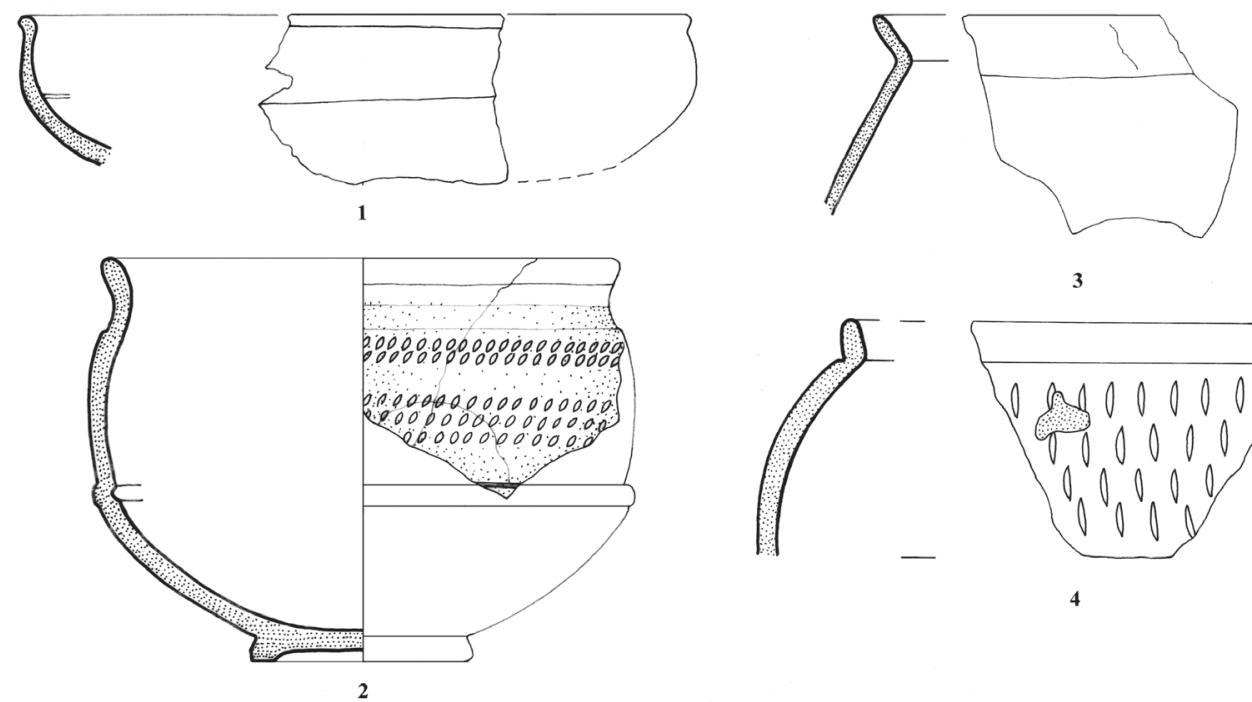

3
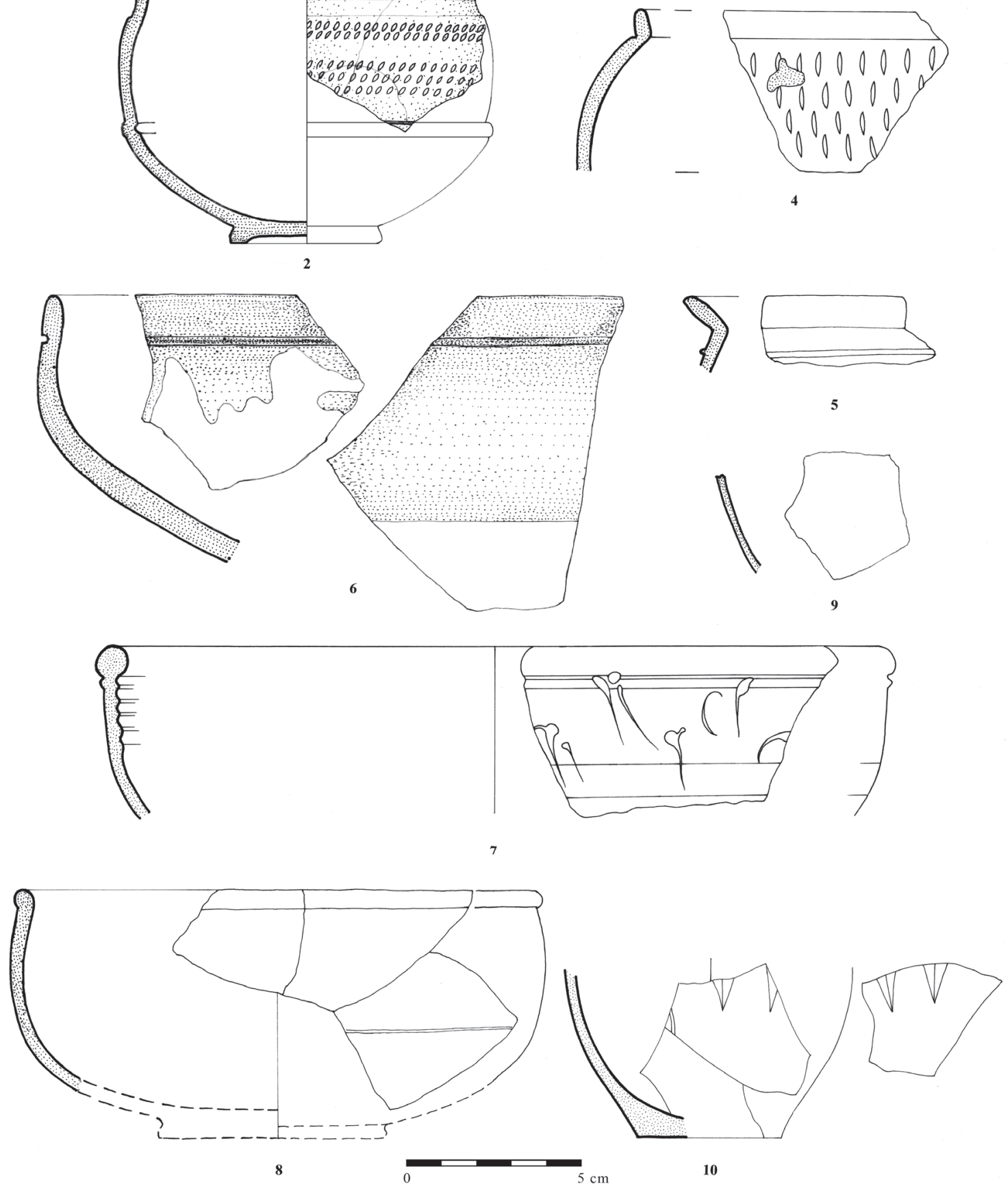

Fig. 4. Ceramica a pareti sottili dalla cisterna scavato nel centro del cortile settentrionale e dal canale che vi conduce, nonché dallo strato d'incendio della villa più antica. 
35. Taglio A2, riempimento del fosso del muro, a 50-60 cm. Scodella carenata a parete leggermente arrotondata, tendente a una forma di patera, più schiacciata, a labbro introflesso; decorazione alla barbottina con motivo di foglie peduncolate, più o meno raggruppate. Tracce di decorazione a rotella. Ricomposta da numerosi frammenti con alcune lacune. Corpo ceramico ocra, ben depurato, verniciato. Vernice marrone arancione. ${ }^{81}$

Pubblicato da: Gabler-Redő 1986, 74, Nr.16, Fig.34,4

36. Nell'ambiente verso Ovest dall'ingresso principale della villa (5/10/1998) è stata rinvenuta un'olletta ricomposta da 9 frammenti; molti altri appartengono a un vaso simile a corpo globulare. Orlo a mandorla incavato internamente, con superficie esterna convessa $^{82}$. Profilo esternamente convesso .v. Olcese 2003, tav. VIII .7 Corpo ceramico rosso, ruvido con inclusioni micacei, parete interna annerita, orlo annerito. ${ }^{83}$ (Fig. 5.4)

Fine del I sec. -III sec. d. C.

37. Taglio B+11, (5/10/1995) al di fuori dei muri, riempimento del canale. Coppa emisferica priva di ansa, con orlo leggermente introflesso, forma Ricci 2/403. Sotto il labbro, una solcatura. Piede ad anello con fondo concavo v. Duncan 1964, forma 12, fig.9,48. Argilla color rosso arancione, vernice di colore marrone violaceo ${ }^{84}$. (Fig. 5.5)

38. Taglio h-1, verso l'Ovest dall'ossuarium, sotto il livello del "Westwerk" (29/09/2005).

Fr. di parete di una tazza. Argilla ocra (Fig. 5.6)

39. Taglio j-1, lungo il muro N-S, ad Ovest dal muro sopra lo zoccolo, a 60-80 cm dalla superficie della soglia (13/10/1999). Coppa tendenzialmente globulare. L'orlo arrotondato, leggermente inclinato verso l'esterno. v. Ricci 2/338 Argilla grigia con pareti brunastre. (Fig. 5.7)(gruppo II)

40. Taglio d+11, saggio fuori i muri, accanto all'apertura per il canale, sotto il basamento, crollo (2/10/ 1995).

Ansa a nastro con due solcature ${ }^{85}$. Argilla ocra giallognola, vernice: bruna. I manufatti simili all'esemplare n. 35 all'inizio furono prodotti principalmente nell'Italia Centrale dove si diffusero già nel I sec. a. C. Nelle figline la loro produzione è attestata fino all'età antonina (Fig. 5.8). ${ }^{86}$

Pur essendo quasi uguale la quantità di ceramica a pareti sottili nei due complessi di reperti, tuttavia si può stabilire che, il riempimento della cisterna del cortile $\mathrm{N}$ contiene un numero maggiore di esemplari del tipo databile al II sec., come per es. il boccalino con collarino a forma Marabini XLVI.

\section{CERAMICA COMUNE RINVENUTA NEL POZZO NEL TAGLIO G-4}

Nel riempimento della cisterna abbiamo trovato in tutto 390 frammenti di vasellame. Questo numero, però, comprende anche i frammenti di ceramica medievale trovata negli strati superiori e pubblicata già prima da Á. Kolláth. Il contenuto del contesto comprendeva 5 terre sigillate, 14 esemplari di ceramica a pareti sottili, nonché 10 anfore.

\section{II b Ceramica da mensa}

\section{Brocche}

Si tratta di una tipologia la cui datazione presenta varie difficoltà essendo presente dappertutto dall'età tardo-repubblicana fino al I e II sec. d. C. ${ }^{87}$

1. Brocca con orlo trilobato, con un incavo interno in corrispondenza del beccuccio. Tracce dell'ansa impostata sotto l'orlo, tipo Olcese $5 . .^{88}$ (gruppo I a)

Argilla ben depurata, marrone rossastra.

${ }^{81}$ DunCAN 1964, 42; M.G. MAIOLI: Vasi a pareti sottili grigie dal Ravennate. Acta RCRF 14-15 (1972-1973) 121, 28.

${ }^{82}$ C. Annese-G. De Felice-M. Turchiano: Ceramiche della prima e media età imperiale dai riempimenti delle latrine della domus A. Ordona X. (2000) tav.VII, 10.5; C. Bonnet-C. Batigne Vallet: Contribution à l'étude des céramiques grises kaolinitiques voconces d'après le mobilier issu des fouilles de tracé drômois du TGV Méditerranée. Acta RCRF 37 (2001) 79. fig. 3,6.
${ }^{83}$ S. MASSEROLI: La ceramica a pareti sottili. In: R. Invernizzi (a cura di):...et in memoriam ceorum. La necropoli romana dell'area Pleba di Casteggio, 2010, Tav.III.6.

${ }^{84}$ S. Menchelli-M. Pasquinucci: Ceramiche con rivestimento rosso nella Tuscia settentrionale. Acta RCRF 42 (2012) fig. 3,14.

${ }^{85}$ FAGA 2008, fig. 3,2.

${ }^{86}$ Masseroli 2010, 144.

${ }^{87}$ C. Pavolini: Scavi di Ostia XIII. La ceramica comune. Le forme in Argilla depurata dell' Antiquarium. Roma 2000, 149-150.

${ }^{88}$ OlCese 2003, tav.XXVIII.2; Vegas 1968, Abb.14,141. 

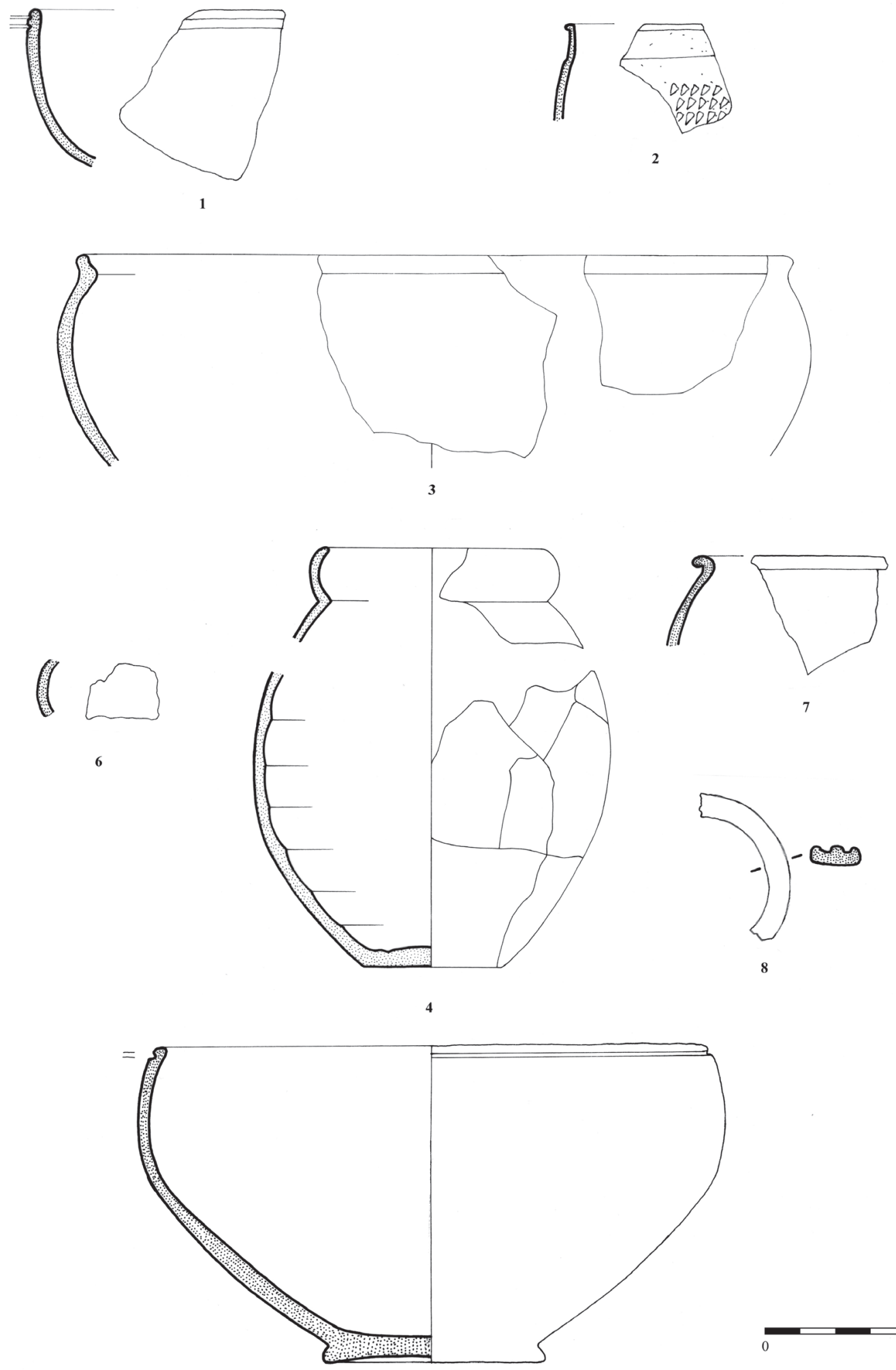

0

Fig. 5. Ceramica a pareti sottili rinvenuta nello strato d'incendio della villa più antica e in siti stratigraficamente indefinibili (fuori contesto). 
2. Fr. di una brocca con corpo piriforme, tipo Olcese $2^{89}$. A questa brocca appartengono 17 frammenti, tra gli altri il punto di impostazione dell'ansa. La forma è nota nei sec. I e II, ma ebbe maggior diffusione del I sec. Fu prodotta a Sutri e anche sul Gianicolo. ${ }^{90}$ Argilla rosso mattone all'esterno, all'interno la superficie di frattura è di color grigio (Fig. 6.1)

3. Ansa costolata da due solchi. Argilla marrone grigiastra (Fig. 6.,2)

4. Fr. del collo di una brocca ${ }^{91}$ Forma Sutri 32. Argilla rosso-giallastra, poco digrassata con sabbia (Fig. 6.3).

5. Fr. del fondo di una brocca tipo Sutri 58. Argilla rosso-giallastra ${ }^{92}$

6. Ansa a nastro di una brocca. Argilla rosso-giallastra, la superficie di frattura è grigia (Fig. 6.,4)

7. Orlo inclinato verso l'esterno, di una brocca, unito alla parete da un gradino. L'orlo è accentuato incavi esterni. Dm: $8,6 \mathrm{~cm}$ Argilla rosso-giallastra. (Fig. 6.5)

8. Fondo di una brocca. Argilla giallo-rossastra chiara, con mica sporadica

9. Fondo di una brocca con piede ad anello ${ }^{93}$ di forma Sutri 36. Argilla all'esterno rossa, con superficie di frattura grigia (Fig. 6.6)

10. Fr. di parete di una brocca con un listello di forma Sutri 37. Argilla giallo-rossastra ${ }^{94}$ (Fig. 6.7)

11. Parete di una brocca a profilo arrotondato. Argilla marrone giallastra, digrassata con sabbia

12. 3 frammenti di una brocca a pareti spesse. Argilla con superficie di frattura rossa, all'esterno scolorita, ben cotta.

13. 9 frammenti di una brocca. Argilla rosso-giallastra, la superficie di frattura è sporadicamente grigia, all'interno digrassata con granelli di sabbia

14. Fondo di una brocca-di forma Sutri 56. Argilla rosso-giallastra, nera all'interno (Fig. 6.8)

\section{Olpai}

Questa tipologia si trova anche nella Campania, ma a Pompei non è frequente ${ }^{95}$. Il materiale rinvenuto a Gabii proviene dall'età tardo-repubblicana, mentre ad Ostia, nel materiale delle a Terme del Nuotatore è venuta alla luce in un contesto di età flavia. ${ }^{96} \mathrm{G}$. Gasparetti azzarda anche una datazione più tarda ( fine del sec. I e inizio del sec.-II). ${ }^{97}$

15. Olpe con orlo a fascia distinto, ingrossato, arrotondato, internamente concavo. Collo alto, stretto cilindrico, ansa a nastro, scanalata, impostata nel collo. ${ }^{98} \mathrm{Dm}: 6,6 \mathrm{~cm}$. Argilla rosso, marrone (Fig. 7.1).

\section{Coppe}

16. Orlo di una coppa emisferica con orlo ingrossato e un listello sotto il labbro di forma Sutri 7. Argilla rosso arancione, con vernice marrone, molto consumata. L'interno è quasi nero. ${ }^{99}$ (Fig. 7.2)

17. Orlo di una coppa emisferica con orlo tagliato obliquamente di forma Sutri 6. Argilla rosso-giallastra (Fig. 7.3)

18. Orlo di una coppa emisferica? con orlo obliquamente tagliato. Argilla rosso-giallastra, all'interno la vernice rosso-arancione è scrostata.

${ }^{89}$ Olcese 2003, tav. XXV.1.

${ }^{90}$ Olcese 2003, 93-94; M. Vegas-A. Martín López: Ceramica comun y de paredes finas. In: M. Almagro-Gorbea: El santuario de Juno en Gabii. Excavaciones 1956-1969. Roma 1982, 474, fig.9.

${ }^{91}$ A. Carbonara-G. Messineo: L Celsa (circ.XX) Bollettino della Commissione Archeologica di Roma 1991-1992, 250,22.

${ }^{92}$ Duncan 1964, fig. 17,209; C. M. Coletti-C. PAVOlini: Ceramica comune da Ostia. In: Les céramiques communes de Campanie et de Narbonnaise. Actes des Journées d'étude organisée par le Centre Jean Bérard et la Soprintendenza Archeologica per le Province di Napoli e Caserta (sous la direction M. Bats). Naples 1996, 397, fig. 5,1 .

${ }^{93}$ DunCAN 1964, fig. 14,146.

${ }^{94}$ DunCAN 1964, fig. $14,156$.

95 OLCESE 2003, 96.

${ }^{96}$ Ostia IV. Le Terme del Nuotatore. Scavo dell' ambiente XVI e dell'area XXV. (a cura di A. Carandini-C. Panella). Studi miscellanei 23. Roma 1977,349, tav.LXI, fig.491.

${ }^{97}$ Gasparetti 1996, 40.

${ }^{98}$ Olcese 2003, tav.XXX.2; Gasparetti 1996, fig.6,35.

${ }^{99}$ DunCAN 1964, fig. 8,41 . 

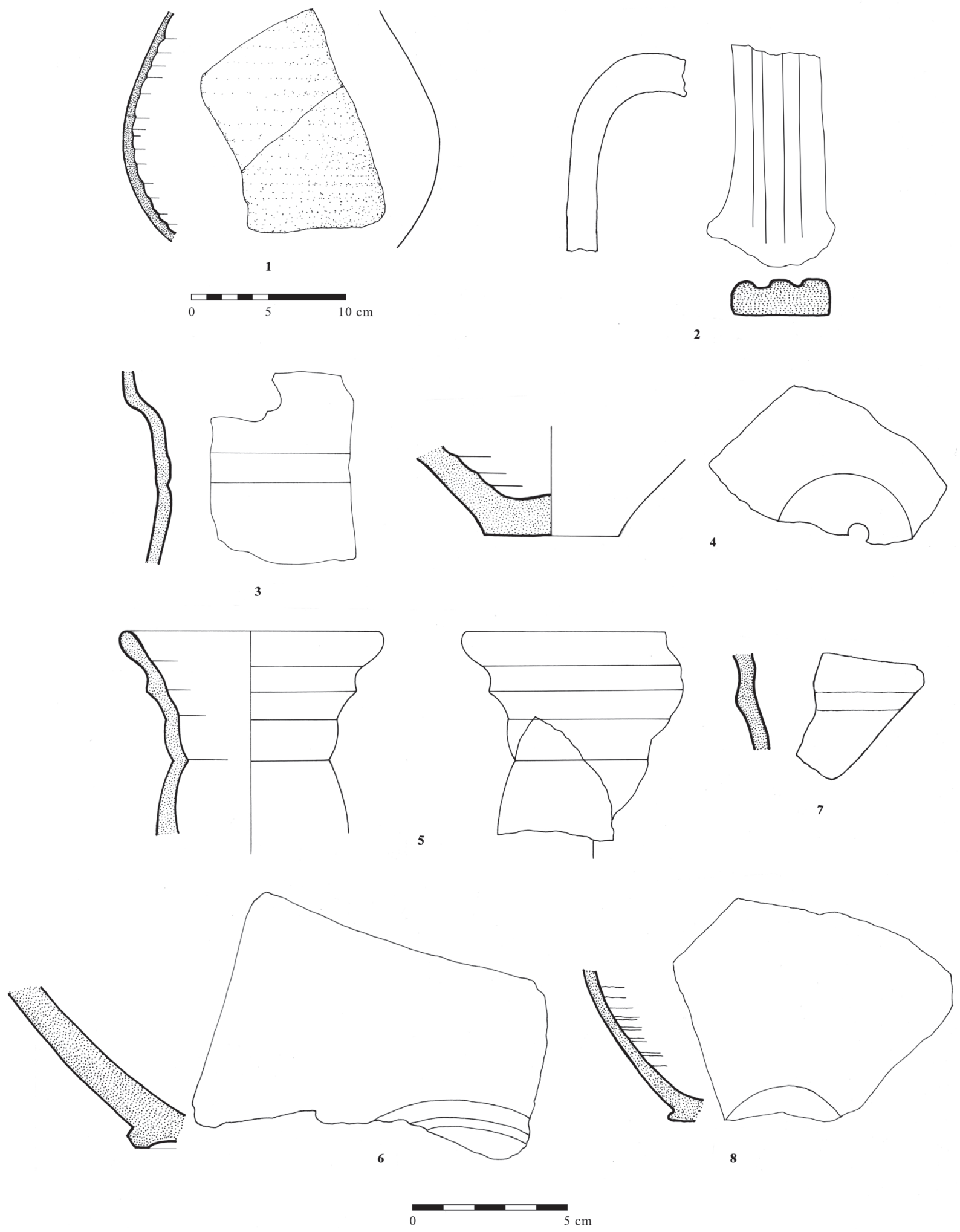

Fig. 6. Brocche dal pozzo trovato nel taglio g-4.

Acta Archaeologica Academiae Scientiarum Hungaricae 66, 2015 


\section{II c Ceramica da fuoco}

\section{Pentole}

12 o 13 esemplari delle 45 pentole in tutto, possono essere identificati come pentole con orlo a tesa appartenente alla forma Olcese $3 \mathrm{~b}$. La maggior parte dei reperti è stata ritrovata nel riempimento della cisterna, a 350-370 cm di profondità. Abbiamo trovato in questo materiale anche un esemplare al quale appartenevano 9 frammenti e poté essere ricostruito quasi perfettamente.

19. Pentola con orlo a tesa. L'orlo è quasi completamente diritto, a sezione quadrangolare e presenta scanalature. La parete quasi diritta è leggermente inclinata verso l'interno; la carena è poco accentuata. Parete inferiore leggermente convessa. Sulla spalla si vede una solcatura orizzontale. Pasta marrone nerastra all'esterno, all'interno colore rosso-mattone (Munsell 2.5-YR 5/6-5/8). La superficie di frattura all'esterno è rossa, all'interno grigia. Alcuni granuli di quarzo, piccole inclusioni di mica. L'orlo bruciato è nero. Dm: 24,2 cm, altezza: 11,6 cm, fondo Dm: 6,6 cm. Dal punto di vista tipologico può essere ritenuta in parte di forma 2211 c, questa variante è frequente negli anni intorno al 100 d. C. ${ }^{100}$ (Fig. 7.4)

I contorni delle pareti laterali di uno dei frammenti sono tendenzialmente globulari mentre nelle altre varianti troviamo pareti quasi verticali, o con parte superiore leggermente introversa. Anche nel caso di questa variante sulla spalla si osserva una solcatura orizzontale.

Forse si può inquadrare a questo gruppo un vaso con orlo più sottile e introverso, mentre la parete laterale è nella parte superiore leggermente inclinata verso l'esterno e la parte sottostante poco arcuata c'inclina verso l'interno, formando in tal modo un contorno tendenzialmente globulare. Sul lato di questo vaso si trova pure una solcatura divisoria orizzontale. Tra gli altri frammenti abbiamo trovato varianti grigiastre, brunastri e giallastri, però l'orlo anche in questi casi rimane nero. Il diametro ricostruibile degli altri vasi oscilla tra i cm 26-27,2.

Pentola da fuoco, generalmente caratteristico nei primi sec. dell'età imperiale: la denominazione antica sarà stata caccabus. Apicio la menziona parlando della cottura o ripassatura dei cibi. M. Bats la ritiene di derivazione punica passata poi ai greci. ${ }^{101} \mathrm{~A}$ Pompei questo tipo di pentola è documentato dal II sec. a. C.; era in uso nell'ultimo periodo delle città vesuviane ${ }^{102}$ e conobbe la sua maggior diffusione nell'Italia Centrale e Meridionale nei secoli I e II d C. M. Vegas sostiene che derivasse dai tegami, di forma simile nel II sec. a. C. ${ }^{103}$ Uno dei luoghi di produzione nel terzo quarto del I sec. a. C. probabilmente fu Sutri ${ }^{104}$. Per la sua produzione in periodi precedenti abbiamo solo pochi dati a disposizione. ${ }^{105} \mathrm{Il}$ tipo fu estremamente diffuso in tutto il Mediterraneo, specialmente in Occidente. Nonostante l'orlo a tesa variamente sagomato, nel periodo che va dal II sec. a. C. al II sec. d. C. non si osserva una ${ }^{106}$ diversificazione evolutiva. La produzione e l'esportazione di questa tipologia ha avuto inizio anche nell'Etruria circa nel I sec. a. C. Si conoscono numerosi confronti derivanti dall'Italia Centrale, da Ostia ${ }^{107}$, dal materiale rinvenuto nella Casa delle Pareti Gialle, da Mola di Monte Gelato e dal Poggio del Capitano, nonché dalla Villa dei Quintili ${ }^{108}$ di Roma. Si trovano analogie anche più distanti a Gabii ${ }^{109}$, Cosa ${ }^{110}$, Albintimilium ${ }^{111}$, Sutri ${ }^{112}$ e Settefinestre $^{113}$, e anche a Roma, nel complesso di reperti della figlina La Celsa situata lungo la via Flaminia ${ }^{114}$, nonché nel

${ }^{100}$ V. Di Giovanni: La ceramica da cucina nella Campania romana. In: Les céramiques communes de Campanie et de Narbonnaise. Actes des Journées d'étude organisée par le Centre Jean Bérard et la Soprintendenza Archeologica per le Province di Napoli e Caserta (sous direction M. Bats). Naples 199684.

${ }^{101}$ M. BATS: Vaiselle et alimentation à Olbia de Provence (v. 350-v.50 av.J.-C). Modèles cultureles et catégories céramiques. Revue archéologique de Narbonnaise suppl.18. Paris 1988, 69.

${ }^{102}$ A. BRUCKNER: Küchengeschirr aus der Casa del Fauno in Pompeji. Acta RCRF 7 (1965) 9, fig.2,n.9; V. Di Giovanni: La ceramica da cucina nella Campania romana. In: Les céramiques communes de Campanie et de Narbonnaise. Actes des Journées d'étude organisée par le Centre Jean Bérard et la Soprintendenza Archeologica per le Provice di Napoli e Caserta (sous la direction M. Bats). Naples 1996, 82. 103 Vegas 1968, 38.
${ }^{104}$ DunCAN 1964, tipi 20-24.

105 Olcese 2003,39.

106 Di GiovanNi 1996, 82-84.

${ }^{107}$ Ostia II. fig.478; Ostia III. 454.

108 Olcese 2003, 76.

109 Vegas 1968. Abb. 15,148.

${ }^{110}$ Dyson 1976, fig.43, 10, tipo 22II.

${ }^{111}$ N. LAmBogLiA: Gli scavi di Albintimilium e la cronologia della ceramica romana. Bordighera 1950, 57, 103, 107.

${ }^{112}$ Duncan 1964, fig. 10, 68, 76, 82-83.

${ }^{113}$ Settefinestre 1985, Tav.24, 13/14.

${ }^{114}$ A. Carbonara-G. Messineo: Ceramica dalle fornaci della Celsa. In: G. Messineo: La via Flaminia da Porta del Popolo a Malborgehtto Roma 1991, fig. 248,6. 

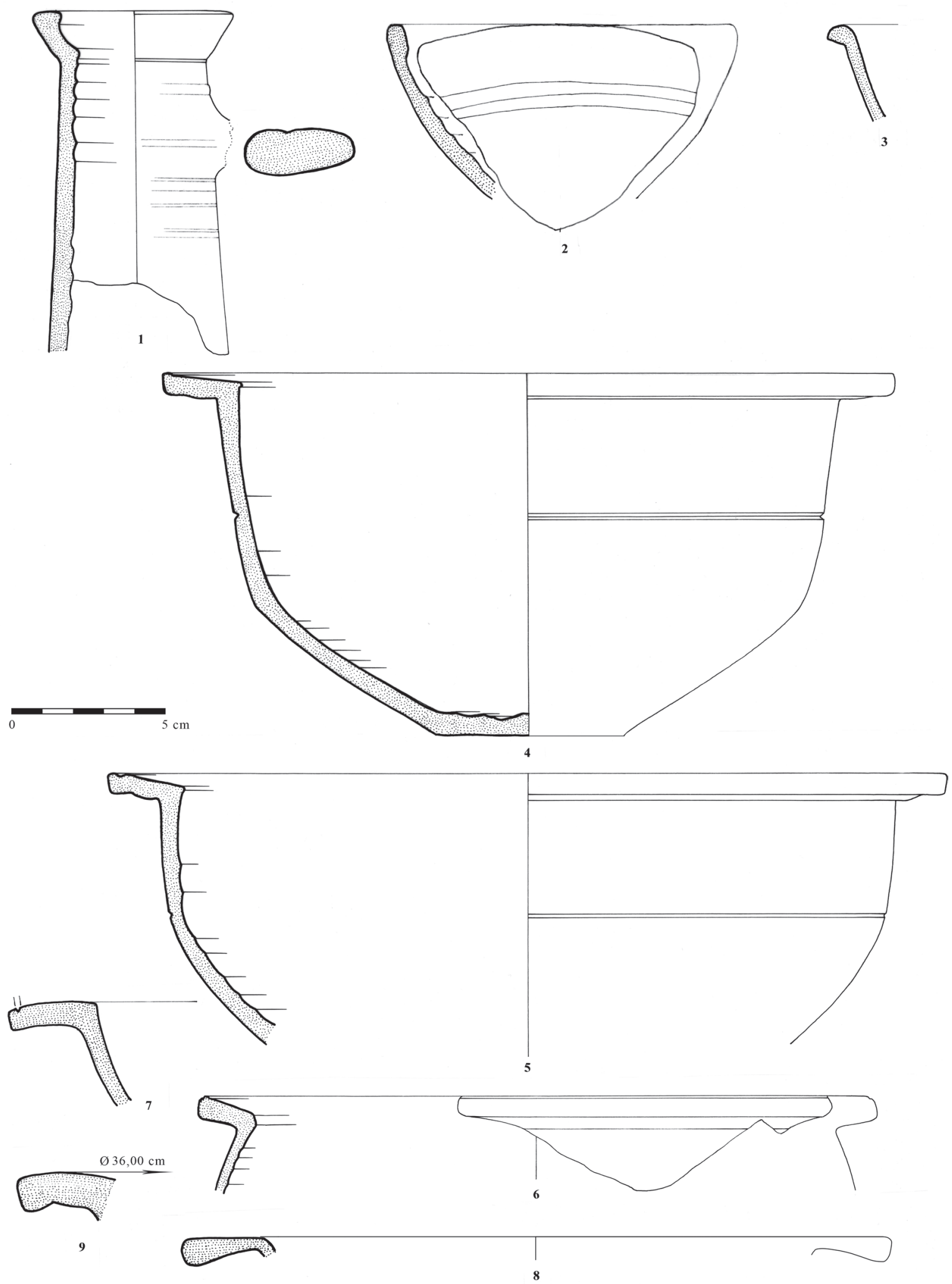

Fig. 7. Olpai, coppe e pentole dal pozzo rinvenuto nel taglio g-4. 
sito di età augustea ubicato sul versante settentrionale del Palatino e a Vasanello ${ }^{115}$. Questo tipo conobbe ampia diffusione oltre che in Campania, anche della parte E. dell'Italia Meridionale ${ }^{116}$. In Spagna e 1'Africa del Nord si conoscono i tipi di fabbricazione locale, ma compare anche a Corinto e Creta ${ }^{117}$. Le varianti venute alla luce a Ordona sono di colore grigio-tendente al bruno, similmente ai nostri reperti, ma anche in questo caso l'orlo è annerito. Abbiamo potuto individuare i frammenti della forma Olcese $3 \mathrm{~b}=$ Annese tipo 5.1 sin dall'inizio dei nostri lavori di scavo eseguiti a San Potito, questi però erano comparsi in uno strato di crollo stratigraficamente non valutabile ${ }^{118}$. Per la cronologia abbiamo a disposizione diversi dati, essendo il tipo frequente a Ostia, nel periodo che va dall'età flavia fino all'inizio del regno di Adriano (90-140 d.C. $)^{119}$, d'altronde il tipo appare anche nel relitto di nave affondata a metà del I sec. d. C., ritrovato a Dramont. In base al contesto venuto alla luce dalla cisterna di San Potito in cui trovammo una moneta coniata nel 101/102 permette di azzardare una simile datazione.

Le proporzioni di tale forma sono conosciute solo dai contesti ostiensi in cui vennero analizzate anche le quantità delle tipologie ritrovate. ${ }^{120}$

20. La pentola molto frammentaria di cui si è conservata solo la parte superiore con orlo a tesa provvisto di una solcatura e leggermente inclinato verso l'interno, potrebbe forse essere un'analogia del vasellame di cui sopra. Le pareti laterali sono sottili e leggermente inclinate, l'impasto e giallo-rossastro con inclusi di calce. Il Dm dell'orlo è di 22,6 cm. (Fig. 7.5)

Somiglia più che altro al tipo Olcese 6 , se ne conosce un ritrovabile a Bolsena ${ }^{121}$. Analogie più lontane comparvero a $\operatorname{Cosa}^{122} \mathrm{e}$ Sutri. ${ }^{123}$

21. Due frammenti del materiale ritrovato nel pozzo possono essere individuati come appartenenti al tipo Annese 5.5/5.3, mentre un esemplare più o meno intero è stato ritrovato nel materiale portato alla luce dalla cisterna scavato nel centro del cortile settentrionale.

Pentola con orlo a tesa, faccia superiore piana e inferiore, leggermente convessa. La carena è accentuata. Attacco con la parete sottolineato da uno spigolo. Fondo piano.

I due frammenti di orlo sono di coloro ocra, la superficie frammentaria è grigia. L'argilla è stata digrassata con sabbia. Siamo riusciti a determinare il diametro di solo uno degli esemplari che risulta essere $\mathrm{cm} \mathrm{11,8.}$

Il tipo si diffuse nel periodo che va dall'età flavia al III sec.; a Ordona venne alla luce dagli strati dei sec. II-III $^{124}$. Si conoscono paralleli rinvenuti ad Ostia ${ }^{125}$, Cosa ${ }^{126}$, Settefinestre ${ }^{127}$ a Luni ${ }^{128}$

22. Pentola con orlo a tesa, con incavo interno di tipo Olcese $6=$ Bolsena 455. Dm: 22,6 cm. Argilla giallo-rossastra, con inclusioni di calce. ${ }^{129}$ (Fig. 7.6)

23. Pentola con orlo leggermente arrotondato, sull'estremità una leggere solcatura. Parete verticale leggermente arrotondata. ${ }^{130}$ Argilla giallo-grigiastra e brunastra (Fig. 7.7)

24. Pentola con orlo ingrossato ${ }^{131}$. Argilla giallo-arancio (Fig. 7.8)

25. Fr. di una pentola a parete diritta di forma Sutri 21. Argilla giallo-arancio, con macchie di vernice arancione ${ }^{132}$

26. 5 frammenti della parete di una pentola a profilo arrotondato di forma Sutri 22. Argilla giallo-rossastra con macchie nere sparse ${ }^{133}$ (Fig. 7.9)

115 OLCESE 2003, 39.

${ }^{116}$ C. Annese-G.De Felice-M. Turchino: Ceramiche della prima e media età imperiale dei riempimenti delle latrine della domus. Ordona X. Ricerche archeologiche a Herdonia (a cura di C. Volpe) Roma 2000, 259- tipo 5.1.

${ }^{117}$ Di Giovanni 1996, 84.

${ }^{118}$ D. GABLER-F. REDŐ: Gli scavi nella villa romana a San Potito di Ovindoli (L'Aquila) (1983-1984. ActaArchHung 38 (1986) 64 , fig. 36,3 .

119 OLCESE 2003, 40.

${ }^{120}$ OLCESE 2003, 27-28.

${ }^{121} \mathrm{La}$ Citerne 5 et son mobilier. Production, importations et consommation III e siècle/debut I er siècle av. J-C. et deuxième tiers du I er siècle ap. J-C. MEFRA suppl. 6 vol.VII. 1995, 178-179, fig.55 n. 455 .

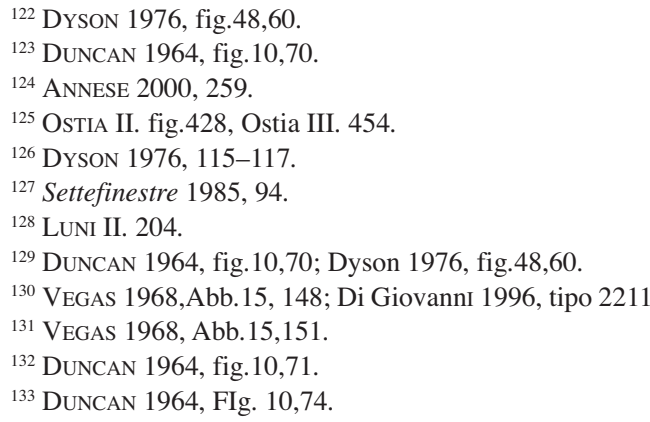


Olle

L'olla (in latino aula) ${ }^{134}$ è la denominazione collettiva di vasi in cui il diametro della bocca è più piccolo di quello della parete laterale, il fondo è piano o articolato in modi differenti. Si tratta di vasi usati prevalentemente sia come vasi da fuoco sia come contenitori in particolare per uva, frutta, noci ecc. ${ }^{135}$

\section{Olla a collarino, tipo Olcese 9}

Questa tipologia può essere qualificata come ceramica da mensa o da fuoco. Si trovano analogie a Ostia ${ }^{136}$, Vasanello $^{137}$, Roma (Curia, Palatino, La Celsa, Villa di Livia, Villa dei Quintili), Settefinestre ${ }^{138}$,Campania, Pompei $^{139}$ és Napoli ${ }^{140}$.

Le analogie ostiensi derivano dall'età dei Flavi e di Traiano; e anche in base agli scavi palatini è databile alla medesima epoca. Il tipo analogo venuto alla luce a Sutri è databile al secondo e terzo quarto del I sec. ${ }^{141}$. A Napoli invece è stato ritrovato in un contesto datato alla fine del I. sec.

Si ipotizza in base alle analisi chimiche eseguite finora che questo tipo fu prodotto a S. di Roma. A La Celsa invece il tipo fu rinvenuto come scarti di officina, attestando la presenza di uno dei luoghi di produzione.$^{142}$

27. Il fr. rinvenuto nel pozzo, la olla a collarino con orlo a tesa breve, verticale, a corpo ovoidale dal dm di $11,3 \mathrm{~cm}$. Argilla marrone scura, digrassata con $\operatorname{sabbia(Fig.~8.1).~}$

28. Olla a collarino con orlo a tesa breve, corpo ovoidale. Sono venuti alla luce due fr. di orlo di questo tipo. Argilla marrone scuro, con orlo annerito.

29. Olla con orlo a tesa, tipo Menchelli $1^{143}$, argilla giallo-rossastra, digrassata con sabbia (Fig. 8.2).

30. Olla con orlo obliquo forma Sutri $20=$ Di Giovanni 2311 b ${ }^{144}$ (Fig. 8.3).

\section{Olla con orlo diritto Olcese tipo 7}

Questo tipo fu prodotto dall'età tardo-repubblicana fino all'età flavia. Fu rinvenuto a Roma, Gabii, Ostia ${ }^{145}$ e Sutri ${ }^{146}$, a Gabii la maggior parte deriva dall'età tardo-repubblicana, a Ostia da contesti dell'età claudia e flavia; a Sutri da materiale databile al terzo quarto del I sec. Quest'ultimi reperti sono fr. di vasi di produzione locale ${ }^{147}$.

31. Olla con orlo diritto, corpo ovoidale. Dm: $10 \mathrm{~cm}$. Argilla rosso-mattone, dura al tatto, digrassata con sabbia(Fig. 8.4).

32. Olla con orlo diritto. Dm: 9,8 cm Argilla rosso-giallastra, rivestita all'esterno e all'interno di vernice rossa. Presso l'orlo, all'esterno è marrone scura.

33. Olla a corpo ovoidale con orlo diritto, quasi orizzontale ${ }^{148}$ di forma Sutri 1. Argilla rosso-giallastra, digrassata con sabbia piuttosto granellosa. (Fig. 8.5).

34. Fondo di un'olla tipo Sutri 1. Argilla rosso-giallastra, all'esterno di color tendente al marrone.

35. Fondo di un'olla tipo Sutri 1. Argilla giallo-scura, digrassata con sabbia ${ }^{149}$ (Fig. 8.6, Fig. 25.4).

36. Parete di un'olla di forma Sutri 1. Argilla grigia, tracce di vernice rossastra sulla superficie.

37. Olla con orlo estroflesso. Orlo a fascia estroflessa, con superficie esterna convessa e interna concava. Corpo globulare. ${ }^{150}$ Argilla di color giallo-rossastro chiaro, digrassata con granuli di sabbia (Fig. 8.7).

38. Fondo di un'olla con corpo ovoidale, forma Sutri $1 .{ }^{151} \mathrm{Dm}: 4,2 \mathrm{~cm}$. Argilla di colore giallo chiaro, digrassata con sabbia (Fig. 8.8)

${ }^{134}$ W. HILGERS: Lateinische Gefäßnamen. Bezeichnungen, Funktion und Form römischer Gefäße nach den antiken Schriftquellen. Beiheft Bonner Jb 31. Düsseldorf 1969, 39, 112, n.43.

${ }^{135}$ Ostia XIII. 221.

136 Ostia II 1969, 91-925, Tav. XX 351-352 (Terme del Nuotatore); Ostia III 1973, 251, Tav. LVIII, Fig. 506 (Taberna dell'Invidioso).

${ }^{137}$ Olcese 2003, Tav. XII, 3.

${ }^{138}$ Settefinestre 1985, tav.30,12

${ }^{139}$ S. DE CARO: La villa rustica in località Villa Regina a Boscoreale. Roma 1994, fig.40, n.118-119.

140 OLCESE 2003, 84.
${ }^{141}$ J.T. PEŇA: Roman-period ceramic production in Etruria Tiberina: a geographical and compositional study. Voll.I-II. Michigan 1987, 251.

142 OlCEse 2003, 84.

${ }^{143}$ MenCHELli 2005, tipo 1.

${ }^{144}$ Duncan 1964, fig. 10,70; Luni II. tav.245,27, K 2148/8

${ }^{145}$ Ostia II. 1969 93, tav.XXI. 367; Ostia III. 1973, 45, tav.

XV. 24.

${ }^{146}$ DunCAN 1964, 61, fi.g12, forma 28

${ }^{147}$ OLCESE 2003, 83.

${ }^{148}$ DunCAN 1964, fig. 7, 15 .

${ }^{149}$ DunCan 1964, fig. 7, 18 .

${ }^{150}$ ANNESE 2000, fig. 10,5.

${ }^{151}$ DunCan 1964, fig. 7,7. 
39. Parete di un'olla di forma Sutri 1. Argilla rosso-giallastra, digrassata con sabbia ${ }^{152}$.

40. Fondo di un'olla con corpo ovoidale. Dm: $8 \mathrm{~cm}$. Argilla ben depurata, rosso-chiara.

41. Fondo di un'olla tipo Sutri 27 ? Dm: 7,2 cm. Argilla rosso-giallastra, all'esterno color marrone scuro, molto granulosa. (gruppo II)

42. Fondo di un'olla. Argilla brunastra all'esterno e grigia all'interno (Fig. 8.9).

43. Olla a collarino con orlo a tesa breve, collo a sezione arrotondata ${ }^{153}$ di forma Sutri 32. Argilla rossa all'interno, marrone all'esterno (Fig. 8.10).

44. Fondo di un'olla. Dm:3 $\mathrm{cm}^{154}$ Argilla giallo-arancio ben cotta, dura al tatto.

\section{Olletta con orlo estroflesso tipo Olcese 13.}

45. Fr. dell'orlo a sezione triangolare, di un'olletta con spalla molto sporgente. ${ }^{155}$ Argilla giallo-rossastra (Fig. 8.11), tipologia diffusa nei sec. I-II. ${ }^{156}$

\section{Olla con orlo a sezione ovale, arrotondata, collo a sezione troncoconica Olcese tipo 8.}

46. Tipo fabbricato sia a Sutri ${ }^{157}$ sia nella figlina La Celsa ubicata lungo la via Flaminia, databile secondo Dramont D alla metà del I sec. ${ }^{158}$ A Sutri fu rinvenuto in un contesto databile al terzo quarto del I sec. Dm: $13 \mathrm{~cm}$. Argilla giallo-brunastra, digrassata con sabbia (Fig. 9.1.)

Olle con orlo estroflesso, internamente concavo. Corpo schiacciato ${ }^{159}$ tipo Sutri 26. Tipo derivante dalla prima età imperiale. Dm: 12,7 cm. Argilla giallo-rossastra (Fig. 9.2).

47. Fondo di un'olla di forma Sutri $26 . \mathrm{Dm}: 7 \mathrm{~cm}$. Argilla di colore rosso mattone scuro e marrone.

\section{Olla con orlo arrotondato, tipo Sutri 27.}

48. Olla con orlo estroflesso, internamente concavo, forma Sutri 28. Di Giovanni 2311 a. ${ }^{160}$ Argilla rosso-giallastra, e grigiastra presso l'orlo (Fig. 9.3).

Olle con orlo a sezione arrotondata, al collo un sottile listello. ${ }^{161}$

49. Olla con orlo inclinato verso l'esterno Olcese tav. XI.8. Tipo rinvenuto ugualmente a Sutri, ${ }^{162}$ Ostia e anche a Roma. ${ }^{163}$ Venuta alla luce in un contesto di prima epoca flavia e antonina.

50. Olla a corpo ovoidale con orlo a mandorla incavato internamente. Dm:13,3 cm. Argilla giallo-rossastra, poco granulosa, con orlo nero.

\section{Olle con orlo inclinato verso l'interno.}

51. Olla ovoidale con orlo leggermente introflesso, incavato internamente. Si conoscono paralleli rinvenuti a Cosa ${ }^{164}$ e Castel Giubileo. ${ }^{165} \mathrm{Dm}: 13,2 \mathrm{~cm}$. Argilla giallo-rossastra, orlo annerito all'interno (Fig. 9.,4).

52. Parete di un'olla. Argilla giallo-rossastra.

53. Fondo di un'olla. Argilla di colore rosso chiaro-giallastro, digrassata con sabbia.

54. Parete di un'olla a profilo di forma $\mathrm{S}^{166}$ tipo Sutri 12. (gruppo I a)

55. Fondo di un'olla. Argilla rosso-tendente al marrone.

56. Parete di un'olla. Le pareti sono leggermente più spesse. Argilla rosso-giallastra, digrassato con sabbia.

57. Parete di un'olla. Pareti leggermente ingrossate. Argilla rosso-giallastra, all'esterno marrone.

58. 7 frammenti di un'olla a corpo ovoidale. Argilla rosso-giallastra, marrone all'esterno, digrassata con sabbia.

59. Fondo e 5 fr. di un'olla. Pareti leggermente ingrossate. Argilla rosso-giallastra, all'esterno marrone, con grossi granuli bianchi.

60. Fondo di un'olla rinvenuto in una profondità di $440-480 \mathrm{~cm}$. Argilla ben cotta dura al tatto, color crema (beige).

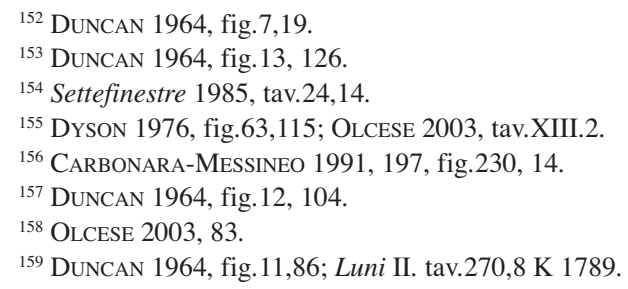

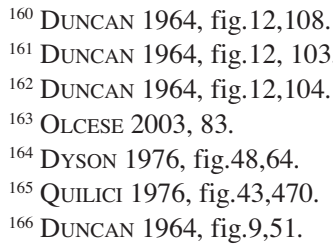



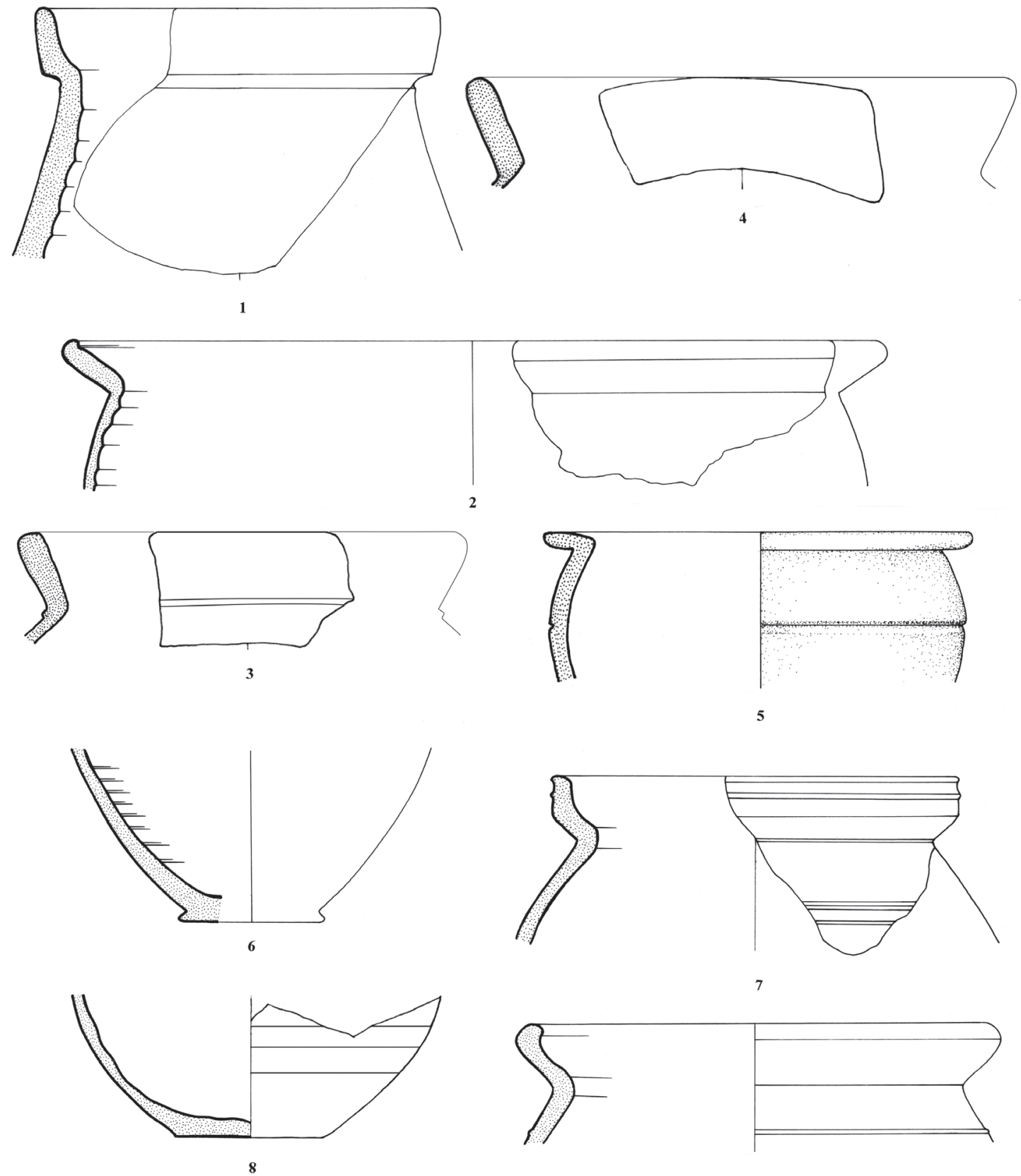

7

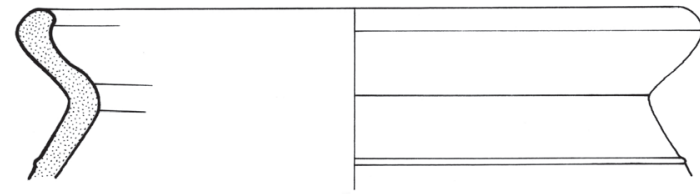

10
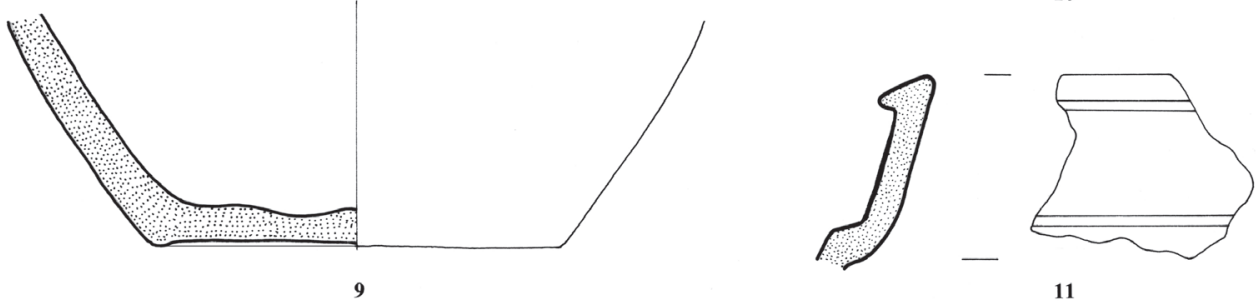

11

Fig. 8. Olle rinvenute nel pozzo del taglio g-4. 


\section{Tegami}

Nel Lazio è diffuso un tipo di tegame con pareti laterali con sezione quasi ad arco e con orlo leggermente rientrante, di cui si conoscono confronti rinvenuti a Pompei, in un contesto databile al secondo e terzo quarto del I sec., a Sutri ${ }^{167}$, mentre a Ostia fanno parte del complesso di reperti derivanti dal II sec. delle Terme del Nuotatore. ${ }^{168}$ Appare anche a Castel Giubileo ${ }^{169}$, La Celsa ${ }^{170}$ sia nel I, sia nel II sec. Gli scarti e i mal cotti di Prima Porta, invece fanno ipotizzare in questo posto un luogo di produzione. ${ }^{171}$ L'analisi di un esemplare derivante da Palestrina permette di presupporre ulteriori luoghi di produzione ubicati a S. e a SO da Roma ${ }^{172}$.

61. Tegame con orlo estroflesso, parete svasata che si restringe verso il basso. Argilla ben depurata, rosso mattone.

62. Fr. di orlo quasi verticale e grosso, a forma Sutri 24. Argilla rossa tendente al marrone, leggermente granulosa (Fig. 10.1)

63. L'orlo verticale tende a ingrossarsi verso l'esterno. ${ }^{173}$. L'argilla rosso-giallastra nella parte esterna dell'orlo è più scura. Si tratta di una forma longeva e ritrovabile nell'Etruria già dal II sec. a. C. ${ }^{174}$

\section{Tegami di tipo Olcese 4}

È un tipo diffuso quasi dappertutto nei sec. I e II. I pezzi venuti alla luce a Ostia derivano dal Lazio e dalla Campania. ${ }^{175}$ Forma ritrovabile anche a La Celsa, contenente relativamente poco materiale di origine vulcanica. ${ }^{176}$ La forma evoca i tipi di ceramica con vernice interna rossa che, erano destinati alla cottura degli alimenti ${ }^{177}$. Questi furono classificati da Ch. Goudineau ${ }^{178}$, e poi fu G. Pucci che, in base ai reperti archeologici e le fonti, ipotizzò che uno dei luoghi di produzione fosse a Cuma. ${ }^{179}$

64. Fondo di un tegame con fondo piano. Argilla rosso-giallastra, all'interno rivestita di vernice rosso-brunastra. Molto digrassata con granelli di calce (Fig. 10.2).

65. Parete di un tegame. Estremità arrotondata e leggermente inclinata verso l'interno. Parete a profilo arrotondato. ${ }^{180}$ Argilla ben cotta, dura al tatto, giallo-rossastra, all'interno tracce di verniciatura rosso-arancione. (Fig. 10.3)

66. Orlo di un tegame con estremità arrotondata e leggermente inclinata verso l'interno. Argilla rossa, all'interno tracce di vernice rossa.

67. Orlo di un tegame a estremità arrotondata, leggermente inclinata verso l'interno. Argilla rosso-giallastra.

\section{Coperchi}

68. Coperchio con orlo distinto rialzato, a profilo arrotondato ${ }^{181}$ tipo Olcese 2. Manico schiacciato, quasi ovale. Tipologia diffusissima probabilmente fabbricata in molti luoghi, l'argilla all'esterno è sporadicamente annerita di colore rossastro - nerastro. (Fig. 9.6, Fig. 25.3)

69. Fr. di un coperchio di forma Sutri 48. ${ }^{182}$ Argilla rosso-giallastra nera, verso l'orlo. (Fig. 9.7)

70. Coperchio con breve manico cilindrico ${ }^{183}$. Argilla scura, marrone-giallastra, la superficie di frattura è nerastra, digrassata con sabbia (Fig. 9.8, Fig. 25.2).

167 PEŇA 1987, 253, fig. 15,3 .

168 Ostia II.308.

${ }^{169}$ L. QuILICI: Saggi di scavo attorno a Fidenae. NotSc 1976, ser.8.ann.CCCLXXIV. XXX, fig.43, 509.

${ }^{170}$ A. Carbonara-G. Messineo: Ceramica dalle fornaci della Celsa in: G.Messineo: La via Flaminia da Porta Popolo a Malborghetto. Roma 1991, fig.247,2.

${ }^{171}$ H. Patterson-A. Bousquet-H. Di Giuseppe-F. FelICI-S. FonTANA-R. WitcheR-S. ZAMPINI: Le produzioni ceramiche nella media Valle del Tevere tra l'età repubblicana e tardoantica. Acta RCRF 39 (2003) fig. 10,2.

${ }^{172}$ Olcese 2003, 87.

${ }^{173}$ L. Gambero: La Liguria costiera tra III e I sec.a.C. Mantova 1999, tav.II.23-24; S. Menchelli Vasi comuni nella Tuscia settentrionale costiera. Aspetti regionali ed apporti mediterranei. Acta RCRF 39 ( 2005) 109, tipo 1.

${ }^{174}$ Menchelli 2005, 109.

${ }^{175}$ Coletti-Pavolini 1996, 407.
176 OLCESE 2003, 87.

177 A.De Bonis-L.Cavanassa-C.Grifa. A. Langella-V. MorRo: Le ceramiche comuni di Cuma. In: Les céramiques communes de Campanie et de Narbonnaise. Actes des Journées d'étude organisées par le Centre Jean Bérand et la Soprintendenza Archeologica per le Province di Napoli e Caserta (sous la direction de M. Bats). Naples 1996315.

${ }^{178} \mathrm{CH}$. Goudineau: Note sur la céramique à engobe interne rouge- pompeien. MEFRA 1970, 159-196.

${ }^{179}$ G. PUCCI: Cumanae testae. La parola del passato. Riv.di Studi antichi 164, sett.-ott. 1975, Napoli, 368-371.

${ }^{180}$ Dyson 1976, fig.46,41; ANNESE 2000, tipo 3.1.

${ }^{181}$ OLCESE 2003, tav.XIX.5.

${ }^{182}$ DunCAN 1964, fig. 15, 178.

${ }^{183}$ C.M. Coletti-C.Pavolini: Ceramica commune da Ostia. In: Les céramiques communes de Campanie et de Narbonnaise. Actes des Journées d'étude organisées par le Centre Jean Bérard et la Soprintendenza Archeologica per le Province di Napoli e Caserta (sous la direction de M. Bats). NAPLES 1996, 405, fig.7,8. 

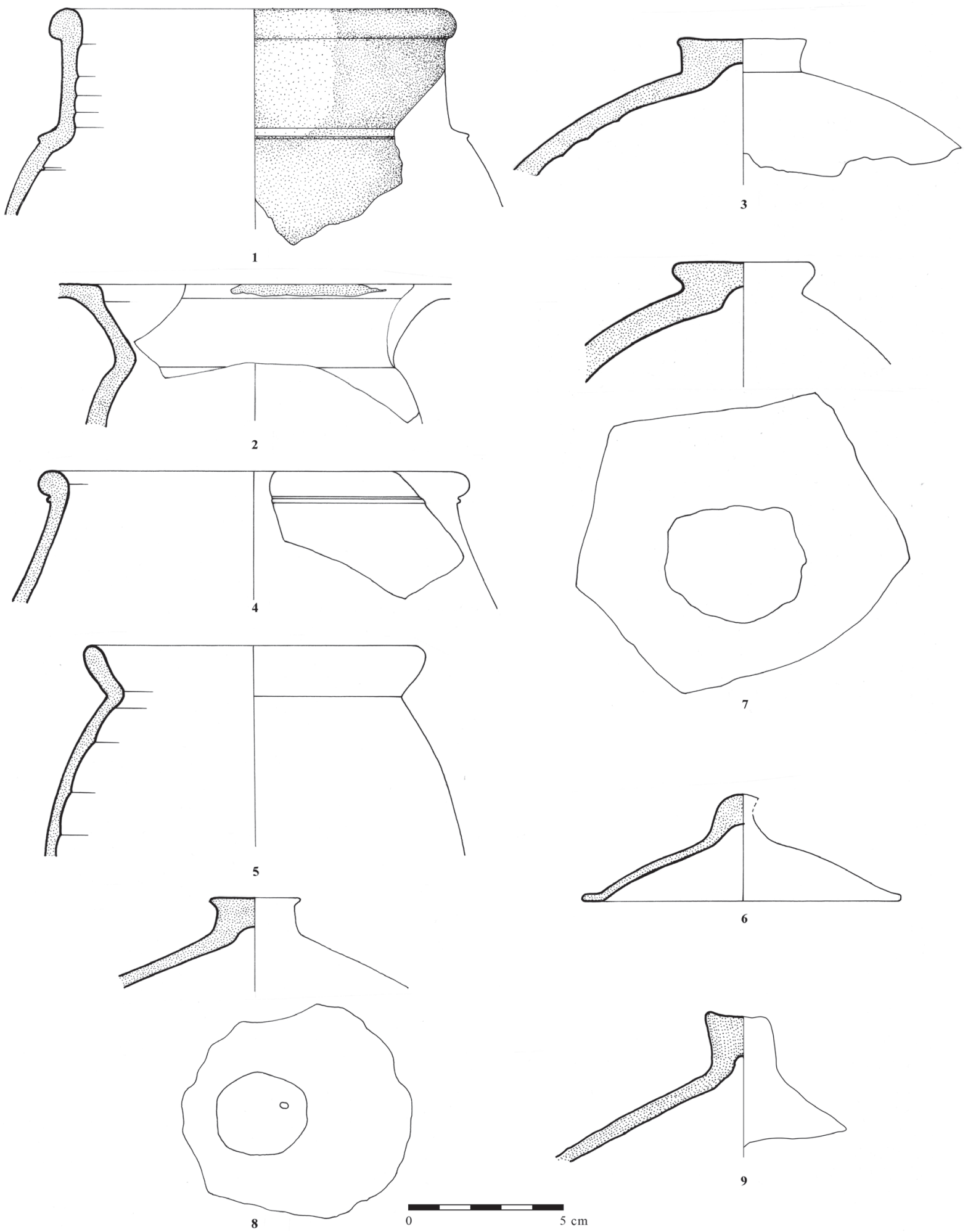

Fig. 9. Olle e coperchi rinvenuti nel pozzo del taglio g-4. 
71. Coperchio con lungo manico cilindrico Sutri forma 48 Argilla giallo-rossastra (Fig.9,9)

72. Coperchio con orlo rialzato a tesa orizzontale, corpo conico di tipo Sutri 48. ${ }^{184}$ Argilla giallo-rossastra (Fig. 9.,5).

73. Coperchio con orlo rialzato a tesa orizzontale, corpo conico. Argilla giallo-rossastra.

\section{II d Ceramica per la preparazione}

\section{Bacino / mortaria}

I precedenti morfologici risalgono ai sec. VI-V a. C. Sono caratteristici gli esemplari privi di inclusi, simili al nostro che, probabilmente serviva come contenitore di sostanze liquide. ${ }^{185}$

I pezzi rinvenuti a Bolsena sono probabilmente di produzione locale. Gli esempi citati da Olcese derivano generalmente dall'età tardo-repubblicana. ${ }^{186}$

74. Mortarium a vasca arrotondata, orlo a profilo a forma di semicerchio. Il bacino presenta le inclusioni del mortarium tipo Olcese 8= Bolsena 433. Dm: 41,6 cm. Argilla marrone-giallastra, con grossi granuli di calce (Fig. 10.4)

\section{Ampolle}

Contenitore di piccole dimensioni utilizzato per profumi, balsami e unguenti quindi soprattutto per scopi cosmetici e farmacologici. ${ }^{187}$ Secondo i rilevamenti stratigrafici degli scavi ostiensi questo tipo è ben documentabile nei complessi archeologici databili al periodo che collega l'età domiziana a quella antonina.

75. Unguentari con corpo piriforme, più allungato ${ }^{188}$. Manca l'orlo. Argilla chiara, color crema biancastra. (Fig. 10.5)

76. Fr. di fondo di un unguentario del medesimo tipo dell'esemplare precedente. Argilla giallo-rossastra, porosa (Fig.10,6).

\section{Incensieri}

Questi incensieri sono definiti nella letteratura archeologica come turibula, incensieri, bacini con orlo decorato o con listello. Ebbero ampia diffusione nell'intero Impero Romano in contesti abitativi e funerari dalla prima età imperiale fino ad epoca tardo antica. Questo tipo di ceramica è ritrovabile sia nell'accampamento militare, sia negli insediamenti civili, nelle case, nei luoghi di culto, negli edifici pubblici o nelle tombe. Se ne conoscono diverse varianti sia per quel che riguarda la forma, le decorazioni e anche le dimensioni.

77. Fr. di un incensiere con vasca quasi emisferica e cordonatura ondulata. ${ }^{189}$. Argilla rosso-giallastra, leggermente porosa, all'interno tracce di combustione di colore marrone-nerastro. (Fig. 10.7,: Fig. 25.7)

78. Fr. di un incensiere con orlo estroflesso, decorato con ondulatura. ${ }^{190}$ Argilla rosso brunastra, porosa. (Fig. 25.6)

Nei siti di rinvenimento di reperti d'epoca romana la vasca di incensiere è una tipologia che ricorre di frequente, pur essendo la sua funzione alquanto discussa ${ }^{191}$, giacché non esiste nessuna fonte antica che, menzioni il turibulum come incensiere di ceramica, cioè come un tipo di vaso con piede che gli archeologi oggigiorno ritengono essere per l'appunto una vasca di incensiere. ${ }^{192}$ (Prima si pensava che il significato fosse mezzo d'illuminazione, piatto da frutta, o vaso di fiori. ${ }^{193}$ ) La denominazione è dovuta al fatto che all'interno, come anche nel nostro

${ }^{184}$ DunCAN 1964, fig. 15, 178.

${ }^{185}$ B. GORI-T. PIERINI: La ceramica comune di argilla figulina. In: Gravisca. Scavi nel santuario greco. Bari 2001, 31.

${ }^{186}$ OLCESE 2003, 104.

${ }^{187}$ Scavi di Ostia XIII. La ceramica comune. Le forme in argilla depurata dell' antiquarium ( C.Pavolini con un contributo di G.Predieri- S.Sfrecola ). Roma 2000, fig.60, 131.

${ }^{188}$ C.M.Coletti-C.PAVolini: Ceramica comune da Ostia. In: Les céramiques communes de Campanie et de Narbonnaise. Actes des Journées d'étude organisées par le Centre Jean Bérard et la Soprintendenza Archeologica per le Province di Napoli e Caserta (sous la direction de M. Bats). Naples 1996, 395, fig.2,4-5.
${ }^{189}$ Luni I, tav.73,34 CM 4444; Dyson 1976, fig.50,104.

${ }^{190}$ DUNCAN 1964,85, fig. $18,233$.

${ }^{191}$ V. MiHAESCU-BîRLIBA: Turibula: concerning the origin and the utilization of a pottery category from the lower Danube. Acta RCRF 33 (1996) 97. Incensieri rinvenuti a Pompei v. A.D’AMBRosio-M. BorRIELlo: Arule e bruciaprofumi da Pompei. Studi della Soprintendenza archeologica di Pompei 3. Napoli 2001.

${ }^{192}$ E. HARsánYi: A füstölőtálak és a füstölés szokása a császárkorban. Antik tanulmányok 50 (2006) 58. (I bacini dei turiboli e l'usanza di bruciare incenso nell'epoca imperiale. Studi sull'Antichità)

${ }^{193}$ HARSÁNYI se ne occupa dettagliatamente in 2006, 59-62 smentendo le relative ipotesi. 


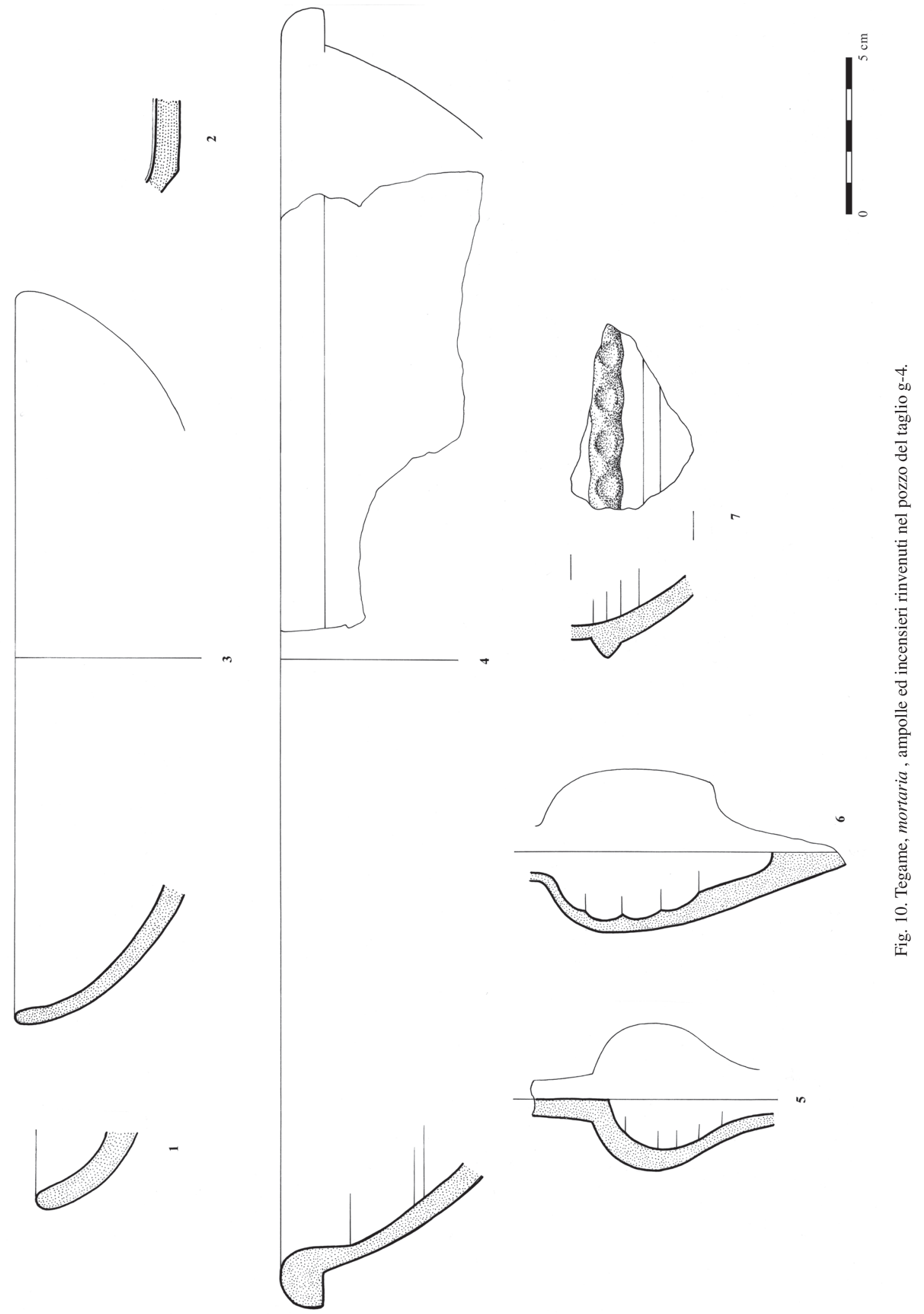

Acta Archaeologica Academiae Scientiarum Hungaricae 66, 2015 
esemplare, si osservano tracce di combustione che trovano spiegazione nella bruciatura di sostanze profumate. Probabilmente furono i greci dell'Italia Meridionale a portarlo in Italia. ${ }^{194}$ In Italia l'uso del turibolo nel culto mortuario o nell'attività di culto non era sconosciuto nemmeno prima. ${ }^{195}$ Sia i confronti di forme, sia i segni esteriori, cioè le tracce di combustione, nonché le iscrizioni votive degli incensieri trovati nelle vicinanze dei templi rivelano che il turibulum servì prima di tutto scopi di culto. A Pompei vennero alla luce diverse vasche di incensieri nei lararium o nelle vicinanze degli stessi. ${ }^{196}$ Nella seconda metà del III sec. il numero di questi oggetti diminuisce decisamente, ${ }^{197}$ probabilmente in relazione alla penetrazione del cristianesimo, siccome i cristiani rifiutavano l'uso del turibolo, ritenuto un rito pagano. ${ }^{198}$

Riassumendo si può costatare che la caratteristica del panorama della ceramica da fuoco consiste nella stragrande maggioranza di prodotti locali per ora non definiti precisamente che, fu completata da tipi di provenienza da aree di incerta localizzazione. La panoramica dell'età flavia e di Traiano pure a Ostia fa supporre che la proporzione della produzione locale o campana fosse quasi di due terzi. Là però, vista la situazione del sito, la proporzione della ceramica comune di produzione africana è notevolmente maggiore, raggiungendo nella fase seguente perfino il $56 \% .{ }^{199} \mathrm{Nel}$ contesto della villa di San Potito fu rinvenuta anche ceramica africana da cucina, ma in una proporzione molto minore rispetto a Ostia. ${ }^{200}$

\section{II e "Imitazione" della terra sigillata italica}

Nel contesto della cisterna situato nel centro del cortile settentrionale è stato rinvenuto un esemplare liscio, inquadrabile alla tipologia dalla forma Consp. 34 .

79. Fr. di una coppa con orlo distinto. Nella parte alta la parete è cilindrica e verticale. Pasta rosso-bruna, senza ingobbiatura. (Fig. 11.1). (gruppo III)

Il tipo spunta anche nel repertorio di forme della ceramica comune; è conforme alla forma 3 della classificazione di G. Olcese. ${ }^{201}$ La coppa a forma Consp. 34 è uno di tipi più diffusi, infatti è frequente a Ostia, Pompei, Luni, Sutri o a Settefinestre. ${ }^{202}$ L'inizio della produzione di questa tipologia può essere datato alla fine del regno di Tiberio, nonché all'epoca claudia. ${ }^{203}$ Pur avendo Ph. Kenrick datato il tipo B 217, simile al nostro, ai decenni trascorsi tra gli anni 35/40 e 80/90 d. C., tuttavia egli aveva anche evidenziato che tale tipo poteva essere inquadrato tra le forme di sigillate italiche più tarde.$^{204} \mathrm{Il}$ gruppo Oct-Pro-Sal che è il più numeroso nel nostro materiale ${ }^{205}$, a Corinto è il secondo per frequenza tra i contesti risalenti alla metà o al terzo quarto del II sec. ${ }^{206}$ La produzione e commercio di questo tipo si è attardata a lungo durante il II secolo non solo nelle province, ma anche in Italia, dove si può notarne l'uso fino alla metà del II secolo. ${ }^{207}$ A San Potito, nel materiale scavato nel taglio g-4 dal pozzo,

${ }^{194}$ C. ZaCCAGNinO: Il thymiaterion nel mondo greco. Analisi delle fonti, tipologia, impieghi. Roma 1998, 78; D’AmbrosioBorRIELlo 2001, 9; H. DobrZANSKA: Early Roman pottery imports from the Northern Pontic area and Asia minor found in Poland- hellenistic traditions and Roman design. Acta RCRF 36 (2000) 339.

${ }^{195}$ HARSÁNYI 2006, 65

${ }^{196}$ D'Ambrosio-Borriello 2001, 70; S. De CARO: La villa rustica in località Villa Regina a Boscoreale . Roma 1994, 34, 179

${ }^{197}$ V. relativamente i pochi esempi di oggetti rinvenuti in area romana: Ceramiche in Lombardia tra II secolo a.C. e VII.secolo d.C. Raccolta dei dati inediti (a cura di G. Olcese). Documenti di archeologia 16 1998, 223 (Milano).

${ }^{198}$ HARSÁNYi 2006, 71-72.

${ }^{199}$ C.M. Coletti-C.PAvolini: Ceramica comune da Ostia. In: Les céramiques communes de Campanie et de Narbonnaise. (sous la direction de M.Bats). Naples 1996,413-414.

${ }^{200}$ V. nota 1.

${ }^{201}$ G. OlCESE: Ceramiche comuni a Roma e in area romana; produzione, circolazione e tecnologia (tarda età repubblica- na-prima età romana). Documenti di archeologia 28. Mantova 2003, 99, tipo 3; ld. még Duncan 1964,57, forma 9.

${ }^{202}$ PuCCI 1985, 397; E. Regoli, Settefinestre 1985, 143.

${ }^{203}$ SCHINDLER-ScheFFENEGGER 1977, 167.

${ }^{204}$ PH. KENRICK: The fine pottery. In: Excavations at Sidi Khrebish, Benghazi (Berenice). III. suppl.to Libya Antiqua 5. Tripoli $1985,158$.

${ }^{205}$ GABLER 2011, 101

206 J.W. HAYES: The Roman Pottery from the South Stoa at Corinth. Hesperia 42 (1973) 445; K. WARNER SLANE: Italian sigillata imports in Corinth. Acta RCRF 35-36 (1987) 195-197.

${ }^{207}$ M.P. LAVIZZARI PEDRAZZINI: Terra sigillata italica e sudgallica. In: Scavi di Luni II. Relazione delle campagne di scavo 1972 1974 (a cura di A. Frova) Roma 1977, 131; S. ZABEHLICKY SCHEFFENEGGER: Terra sigillata tardo-padana. Acta RCRF 31-32 (1992) 422; A. CAGNANO: Un contesto ceramico di età imperiale degli scavi in Palazzo Calissaro di Alba. Quaderni della Soprintendenza Piemonte 12 (1994) 109 

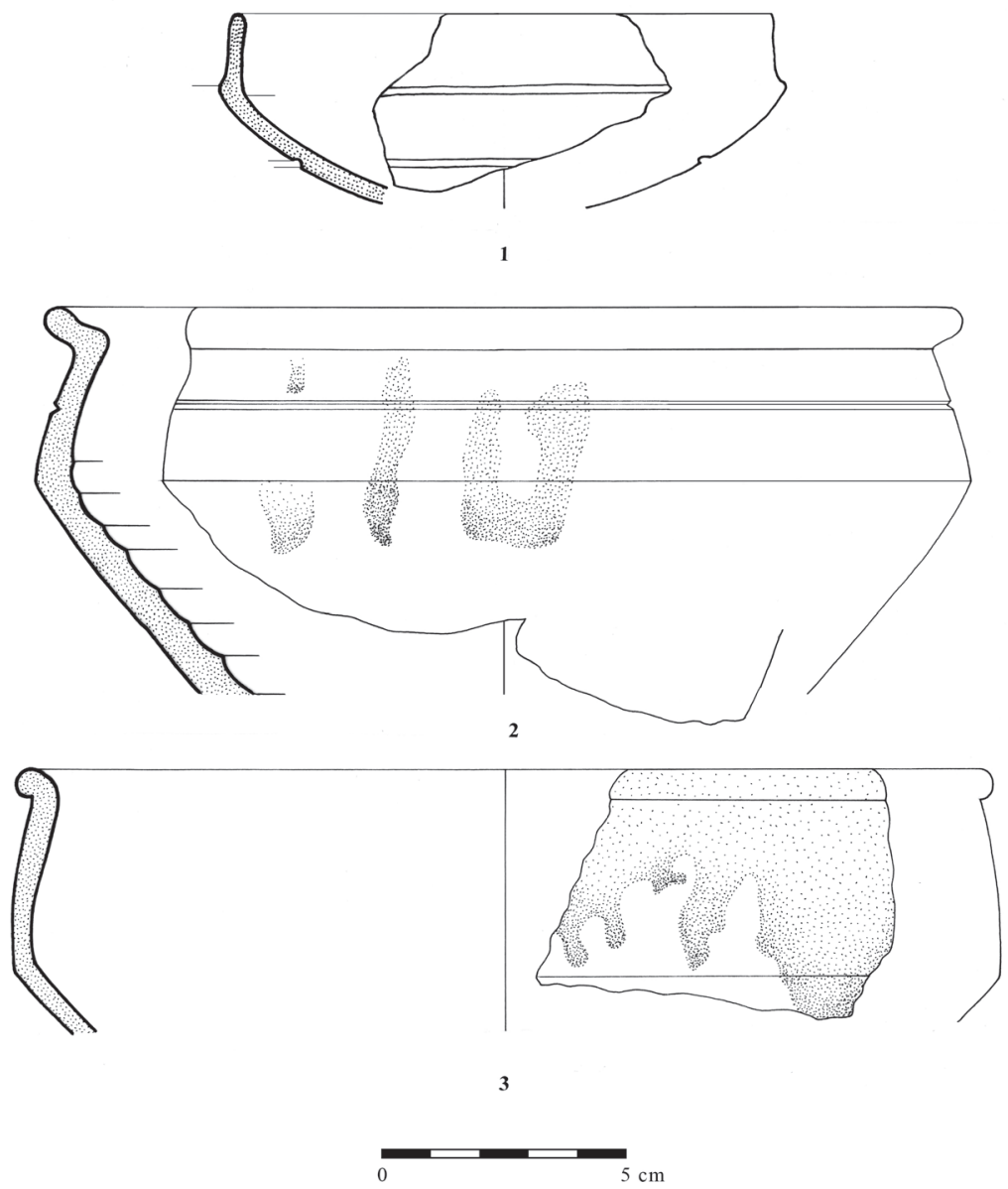

Fig. 11. Imitazione di terra sigillata italica e ceramica con rivestimento.

questo tipo di sigillata è venuto alla luce assieme ad una moneta coniata nel 101/102. ${ }^{208}$ Molti prodotti dell'Italia centrale, come anche i nostri, sono di qualità più mediocre; molte volte la vernice si è conservata solo sugli orli o nelle tracce di tornio. Questa variante è relativamente frequente negli Abruzzi; è venuta alla luce anche nella villa di Grasciano. ${ }^{209}$

Uno dei luoghi di produzione delle cosiddette "imitazioni” delle sigillate italiche forse era a Sutri. ${ }^{210 .}$

\section{II $\mathrm{f}$ Ceramica a rivestimento rosso}

A questo tipo abitualmente s'inquadrano oggetti di ceramica fabbricata generalmente di argilla ben depurata, rossa (Munsell 5YR 7/8), con superficie di frattura granulosa, inclusi bianchi, piuttosto opaca, con rivestimento fine, rosso, arancione o marrone presente soltanto su una parte degli esemplari. Gli esemplari rivestiti non richiedevano processi produttivi molto più complessi rispetto a quelli nudi. Le fornaci in cui veniva cotta la ceramica più semplice erano adatti anche per la cottura della merce rivestita. ${ }^{211} \mathrm{Il}$ colore del rivestimento dipende dalla tempera-

${ }^{208}$ GABLER 2011, 98, Nr. 21 e p. 101.

${ }^{209}$ C.Angeletti-L.Formicone-D. Formicone: La villa romana di Grasciano sec. I. a.C-II. d.C. Mostra archeologica. Museo Civico “G. Romualdi” Notaresco, 2000, p.6 e 8, 10 in basso.
${ }^{210}$ OLCESE 2003, 99.

${ }^{211}$ Menchelli-Pasquinucci 2012, 232. 
tura della cottura. Prima si teneva conto solo delle imitazioni verniciate sigillate, di epoca tarda, ultimamente, invece si è venuti a sapere che, questa merce era reperibile anche in contesti precedenti, databili al I sec. come per es. il materiale scavato nella villa Livia di Prima Porta. ${ }^{212}$ Le produzioni con vernice rossa compariscono nell'Italia Centrale già nella prima età imperiale, in Toscana, nell'Umbria del Sud. Nell'Italia Settentrionale questa tipologia ceramica viene prodotta nelle officine regionali dal II sec. ${ }^{213}$ Nelle zone del Nord si deve tener conto di almeno due centri di produzione, mentre nell'Italia Centro-meridionale si conoscono centri produttivi sul Monte Massico, a Casanova di Carniola e Cascano. Probabilmente la merce ivi prodotta si diffuse solo entro la regione. Nella villa di San Giovanni di Ruoti nei sec. I e II la merce importata dall'Africa prevale su quella locale, mentre nei sec. IV e V la merce locale ha il sopravvento. ${ }^{214} \mathrm{Nei}$ dintorni di Roma, nel materiale derivante dal materiale recuperato dalla fornace sita lungo la Via Flaminia anche fra i tipi di ceramica comune si trovano esemplari con la superficie dipinta completamente o parzialmente. ${ }^{215} \mathrm{~A}$ questo gruppo si può inquadrare un materiale estremamente eterogeneo e non solo dal punto di vista morfologico, ma anche da quello cronologico (Fig. 11.2-3,: Fig. 26.1). Entro i tipi di ceramica comune compaiono le forme ben conosciute dalla produzione di sigillate, tuttavia queste contribuiscono raramente alla datazione come prefigurazioni ispiratrici. C. Corti fa riferimento al problema dell'impianto produttivo della $\mathrm{Ca}^{\prime}$ del Cristo (Castelnuovo Rangone) ubicato nei dintorni di Modena del quale si conosce una fornace situata presso un insediamento rimasto abitato dall'età repubblicana fino ai tempi tardo-antichi. In questo materiale si ritrova il tipo denominato coppa a listello la cui forma rimanda alla Drag.24/25 (Haltern 12; Goudineau 38; Atlante XXXVII; Conspectus 34), ampiamente diffusa tra l'inizio-metà del I e l'inizio-primo quarto del II sec. d.C. ${ }^{216}$ nella produzione di sigillate italiche e dell'Italia Settentrionale. Tuttavia l'esemplare consono alla forma, recuperato nel sito scavato in località Marchesa, proviene dalla frequentazione tarda, inquadrabile tra la metà del IV e la metà del V sec. d. C, mentre un altro reperto derivante dalla zona del Capitolium di Verona è databile al periodo tra i sec. II-III. ${ }^{217}$ Neppure in questo caso la forma, contribuisce alla datazione. Lo stesso dicasi per "l'imitazione di sigillata" presentata più sopra che, però fu rinvenuta in un contesto di alta epoca.

\section{IMPIANTO AL CENTRO DEL CORTILE SETTENTRIONALE}

$\mathrm{Al}$ centro del cortile settentrionale si trova una cisterna rettangolare i cui lati sono di $3,70 \times 3,70$ ed è infossata a 2,40 m sotto la superficie romana. Nei muri della cisterna costruita si di mattoni e pietre risaltano due fili di tubi. Per la costruzione degli angoli furono usati mattoni che offrivano stabilità e precisione, mentre le pietre si trovano solo attorno ai tubi. Da questo deriva il fatto che il muro orientale, dove si trova lo scalino che porta al fondo della cisterna, era costruito solo di mattoni. La cisterna non aveva un pavimento artificiale. Nel livello basso si trova uno zoccolo che sporge a $5 \mathrm{~cm}$ dal muro. Sul livello del pavimento è stato rinvenuto uno strato omogeneo di sabbia alto $2-25 \mathrm{~cm}$ che ovviamente non apparteneva al riempimento della cisterna, ma era il risultato dell'uso del serbatoio. I muri del canale che, parte dall'impianto dirigendosi verso il porticus orientale aderiscono anche al muro orientale della cisterna. La larghezza inferiore di questo canale è di $90 \mathrm{~cm}$, con muri larghi $30-40 \mathrm{~cm}$. I muri non sono adossati con i muri della cisterna, tra di loro c'è un varco. Il canale coperto da mattoni è pure costruito in opus mixtum, mentre i muri sono di pietra e coperti di malta che risultava una superficie liscia. Si poté osservare che i riempimenti di tutti e due gli impianti sono uguali, si tratta di un crollo di costruzione: ornamenti di stucchi di alta qualità, colonne scannellate, capitelli e pezzi di cornici, frammenti di dipinti murali ornamentali, chiodi e ceramiche delle quali in molte si vedono le tracce della pittura. Il riempimento superiore della cisterna formava uno strato duro, pieno di malta e pietre. In base ai rilevamenti possiamo distinguere tre fasi della costruzione della cisterna Nella prima fase fu costruito e usato il sistema di tubi che appartiene al serbatoio. In questa fase non esisteva ancora il canale orientale, ma c'era uno scalino nel lato orientale che serviva alla manutenzione della cisterna. La seconda

${ }^{212}$ A. Ciotola: Ceramica con ingobbio rosso.in: Lacus Iuturnae II. Acta Instituti Finnlandiae 38 (2012) 175.

${ }^{213}$ C. CORTI: Ceramiche a rivestimento rosso della media e tarda età imperiale. In: Insediamento romano della tesa di Mirandola (MO). Ricognizioni e scavi 1930-2011. Quaderni di Archeologia dell'Emilia Romagna 30 (a cura di M. Calzolari-F. Foran). Firenze 2012, 102.
${ }^{214}$ A. Ciotola: Ceramica con ingobbio rosso. In: Lacus Iuturnae II. Acta Instituti Finnlandiae 38 (2012) 175.

${ }^{215}$ Carbonara-Messineo 1991-1992, fig.219; Olcese 2003, 94, tav.26,1-2.

${ }^{216}$ CORTI 2012, 107

${ }^{217}$ IBIDEM. 
fase succedette alle rotture nell'angolo SO che ci fanno pensare ai movimenti sismici che non sono eccezionali nel Fucino. Il danno della cisterna non era notevole, ma il sistema di tubi non poté mai più essere usato. Per questo fu ricostruita la parte orientale della cisterna demolendo lo scalino e congiungendo un canale al muro orientale. Nella fase successiva i due impianti non furono più usati e vennero riempiti del materiale di crollo di costruzione della villa.

Gli scavi rivelano che questo cortile fu costruito nel secondo periodo della villa (dopo Adriano), dunque le tre fasi della cisterna coincidono al secondo, terzo e quarto periodo della villa.

La cisterna nella prima fase segue la simmetria N-S del cortile settentrionale. Nella seconda fase questa simmetria gira di 90 gradi e con il canale che va verso l'ala orientale nasce una nuova costruzione architettonica nel cortile. Il motivo del cambiamento va cercato nel fatto che nel terzo periodo della villa nell'ala occidentale furono usate solo le terme ubicate nell' angolo SO. L'altra parte degli ambienti forse fu distrutta dai movimenti sismici che ha demolito anche il sistema delle tubature, infatti, in quest'area non si vedono le tracce di rifacimenti, che possiamo osservare nell'ala orientale.

Il quarto periodo della villa è caratterizzato dalle tracce di decadenza che segna la fine dell'uso precedente della cisterna che, fu colmata con il materiale del crollo dell' edificio e coperta da uno strato duro di malta e pietre. ${ }^{218}$

Nel riempimento della cisterna nonché del canale sono venuti alla luce in tutto 160 frammenti di vasi, la quantità del materiale ceramico ritrovato nel canale era di gran lunga maggiore di quello derivante dalla cisterna.

Il contesto era costituito da una terra sigillata, il resto erano 10 ceramiche a pareti sottili, 5 anfore e un frammento di lucerna.

\section{III a Ceramica da mensa}

\section{Brocche}

La maggior parte fu rinvenuta in forma molto frammentaria, però i frammenti indicano la presenza di molte tipologie, rendendo veramente difficile la classificazione sia delle brocche, sia delle olpai. ${ }^{219}$ Pur avendo G. Olcese costituito nuclei definiti geograficamente e cronologicamente, tuttavia non si può asserire con sicurezza che la zona degli Abruzzi venisse provvista con merce proveniente dalle officine della città di Roma e dintorni, per cui i limiti cronologici di questo gruppo sono abbastanza vasti partendo dal I sec. per arrivare al III. Abbiamo trovato nel nostro sito pochi frammenti d'imboccature o colli mentre sono venuti alla luce più frammenti di anse a nastro, costolate da solcature, impostate sull'orlo o poco sotto. In vari casi sono poi stati rinvenuti frammenti di corpi ovoidali a fondo piano, convesso o ad anello. I frammenti dei fondi fanno supporre una forma più panciuta e anche più stretta, piriforme, in alcuni casi ricoperta da ingobbio per lo più scadente. La decorazione è rara, si è potuto rilevare solo in un unico caso un motivo a rotella. Sui frammenti abbiamo osservato in vari casi tracce di calce derivanti dal riempimento di detriti.

1. Fr. di orlo con ansa a nastro con due solcature. ${ }^{220} \mathrm{Nel}$ caso di un esemplare di dimensioni maggiori, con ansa di forma simile, non si può escludere l'appartenenza a una brocca biansata. Larghezza dell'ansa: $45 \mathrm{~mm}$. Argilla giallo-rossastra, leggermente porosa (Fig. 12.1).

2. Fr. di una ansa a nastro con due solcature. Larghezza dell'ansa: $35 \mathrm{~mm}$. Argilla ocra giallastra, porosa (Fig. 12.,2).

3. Fr. di parete ed orlo estroflesso con ansa a nastro con una solcatura impostata sull'orlo e sulla pancia. ${ }^{221}$ Larghezza dell'ansa: 18 $\mathrm{mm}$. Argilla rosso-giallastra, porosa. A questo vaso poté probabilmente appartenere anche un frammento di fondo. ${ }^{222} \mathrm{Dm}: 12,4$ cm (Fig. 12.3.)

4. Fr. di un fondo convesso. ${ }^{223} \mathrm{Dm}: 7,9 \mathrm{~cm}$. Argilla rosso-giallastra, ben cotta, dura al tatto (Fig. 12.4).

5. Fr. di fondo piano e parete di forma ovoidale. ${ }^{224} \mathrm{Dm}: 5,9 \mathrm{~cm}$. Argilla rosso-giallastra, porosa, micacea (Fig. 12.5).

${ }^{218}$ GABLER-REDŐ 2008, 57-61.

${ }^{219}$ OLCESE 2003, 93.

${ }^{220}$ Settefinestre, Tav.33,16.

${ }^{221}$ Ostia XIII, fig. 30,44; OlCESE 2003, tav.25,6.
${ }^{222}$ A. MarucCI: Foro Transitorio. Lo scavo dei fori imperiali 1995-2000. Roma 2006, 76, fig.6,17 - questo pezzo risale al II secolo.

\footnotetext{
${ }^{223}$ Coletti-Pavolini, 1996, 393, fig. 1,2; Ostia XIII, fig.30,44.

${ }^{224}$ Dyson 1976, fig.53,138, 22 II.
} 
6. Fr. di fondo ad anello e parete di forma ovoidale. ${ }^{225} \mathrm{Dm}: 9,6 \mathrm{~cm}$. Argilla giallo-rossastra, porosa

7. Fr. di fondo piano. L'impostazione della parete indica una brocca abbastanza larga. Argilla giallo-rossastra, porosa.

8. Fr. di fondo ad nello e parete di forma ovoidale ${ }^{226} \mathrm{Dm}: 10,4 \mathrm{~cm}$. Argilla giallo-rossastra, porosa (Fig. 12.6).

9. Fr. di fondo convesso. L'impostazione della parete indica una brocca abbastanza larga. Dm: 6,6 cm Argilla giallo-rossastra, porosa. Rivestimento rosso scuro, tendente al marrone (Fig. 12.7).

10. Fr. di fondo convesso. Simile a quello di sopra, privo di rivestimento. Dm: $6,9 \mathrm{~cm}$. Argilla rosso-biancastra, sulla superficie residui di calce.

11. Fr. di fondo ad anello. Pareti sferoidali, con incisioni orizzontali. Superficie inferiore con tenue rivestimento rosso. Sulla parete interna tracce di ingobbiatura rossa. ${ }^{227} \mathrm{Dm}: 6,6 \mathrm{~cm}$. Argilla giallastra, leggermente porosa (Fig. 12.8).

12. Fr. di fondo convesso. L'impostazione della parete indica una brocca abbastanza larga ${ }^{228}$ Sulla parete esterna rivestimento rosso, con macchie più chiare. Dm: 6,6 cm. Argilla:giallo-rossastra, leggermente porosa (Fig. 12.9)

13. Fr. di fondo convesso, simile al precedente. Dm:7,1 cm. Argilla rossa e gialla, verso il fondo rossa tendente al marrone, porosa, all'interno inclusioni di calce sparse (Fig. 13.1).

14. Fr. di fondo convesso . Pareti sferoidali ${ }^{229}$ Superficie inferiore rivestita, bruna. Dm: $6,3 \mathrm{~cm}$. Argilla giallo-rossastra, meno porosa (Fig. 13.2, Fig. 27.3).

15. Fr. di fondo convesso, con solcatura formata ad anello. Parete di forma ovoidale. ${ }^{230} \mathrm{Dm}$ : $6 \mathrm{~cm}$. Argilla giallo-rossastra, leggermente porosa. Forse vi appartiene un frammento di spalla con incisioni orizzontali (Fig. 13.3).

16. Fr. di fondo convesso. Dm:5,5 cm. Argilla giallo-rossastra, poco porosa.

17. Fr. di fondo convesso. ${ }^{231}$ Decorazione a rotella. Dm: $5,4 \mathrm{~cm}$. Argilla giallo-rossastra, leggermente porosa. Sul fondo residui di calce. Questo esemplare è stato rinvenuto nel riempimento della cisterna, mentre gli altri vennero alla luce dal riempimento del canale (Fig. 13.4).

18. Fr. di fondo piano. Dm: $5,2 \mathrm{~cm}$. Argilla rosso chiara e giallastra.

19. Fr. di fondo piano. Un frammento di una parete laterale indica un vaso piuttosto largo. ${ }^{232}$ All'esterno tracce di rivestimento rosso, di qualità scadente. Dm: 4,8 cm. Argilla rosso-giallastra., poco porosa (Fig. 13.5)

20. Fr. di fondo piano. Dm: $3,9 \mathrm{~cm}$. Argilla rossa e ocra, porosa

21. Fr. di fondo ad anello ${ }^{233}$ di forma Sutri 55. Argilla rosso-giallastra, porosa.

22. Fr. di fondo piano. ${ }^{234} \mathrm{Dm}: 3,8 \mathrm{~cm}$. Argilla rosso-giallastra, porosa

23. Fr. di fondo piano e parete di forma ovoidale. ${ }^{235} \mathrm{Dm}: 3,6 \mathrm{~cm}$. Argilla giallo-rossastra, porosa

24. Fr. di parete. Lunghezza massima del frammento: $12 \mathrm{~cm}$. Argilla giallastra, porosa

25. Forma ovoidale di brocca costituita di sei pezzi incollati. L: $23 \mathrm{~cm}$. Argilla giallo-rossastra, con strato di calcio polverizzato.

26. Fr. di parete leggermente inarcata. Argilla ocra-giallastra, porosa

27. Diversi frammenti con pareti laterali leggermente inarcate; tracce di rivestimento rosso. Argilla giallo-ocra, poco porosa.

28. Fr. di pareti laterali molto inarcate. L: $14,5 \mathrm{~cm}$. Argilla molto porosa, rosso-giallastra

29. Tre fr. della stessa parete molto inarcata. Argilla ocra-giallastra, molto porosa.

\section{Coppa}

30. In questo gruppo va inquadrato il fr. a forma di goccia di un piccolo piatto emisferico? Argilla giallo-rossastra, porosa (Fig. 13.6).

\section{III $b$ Ceramica da fuoco}

\section{Pentole}

Questo tipo di pentola è alquanto differente dalle pentole rinvenute nel riempimento del taglio g-4, avendo un orlo molto più alto e piegato obliquamente verso l'esterno; all'interno le trace di tornio sono più marcate. A Pom-

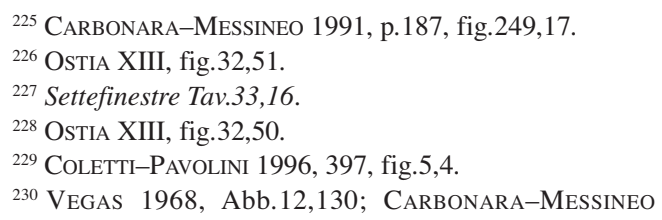
fig. 32,49 .

${ }^{231}$ Coletti-Pavolini 1996, 297, fig.5,1; Ostia XIII,

${ }^{232}$ Ostia XIII. fig. $29,41$.

233 OsTIA XIII. fig. 29,39.

${ }^{234}$ OsTIA XIII, fig. 30, 45.

235 Vegas 1968, Abb.12, 130; Coletti-Pavolini 1996, 396, fig.; OstIA XIII, fig.28,374. 

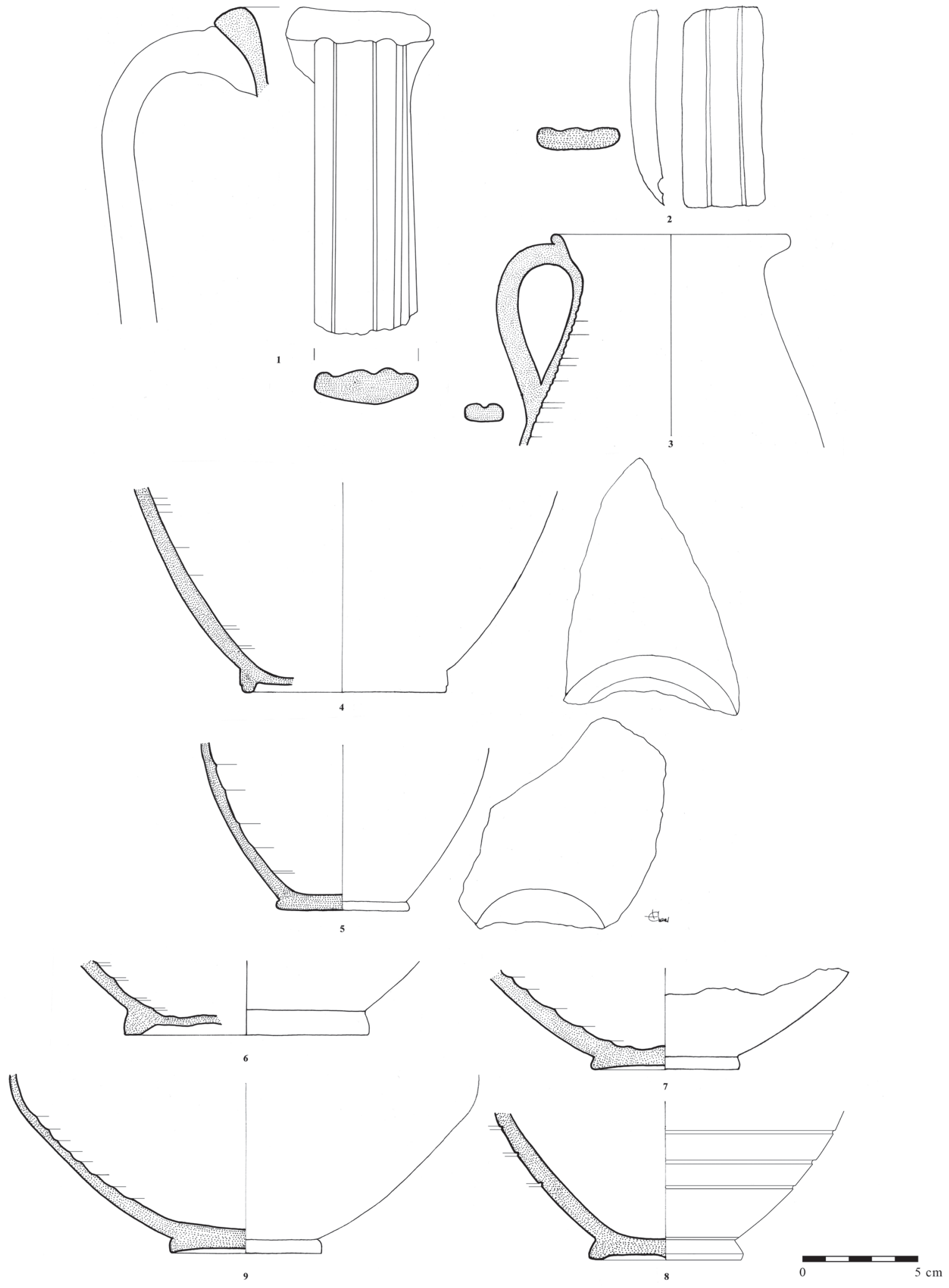

Fig. 12. Brocche rinvenute nel canale conducente alla cisterna nel centro del cortile settentrionale. 
pei sono state rinvenute forme simili (tipo $2211 \mathrm{~g}$ ), ma possono essere identificate come appartenenti a questa variante anche i tipi Olcese 4; quest'ultimi furono prodotti nel I sec. nonché nella prima metà del II sec. Se ne conoscono molte varianti, i centri di produzione potevano trovarsi nell'Italia Centrale, nella stessa Roma e a N. da Roma, forse nei dintorni di Palestrina o Tivoli ${ }^{236}$. Il diametro dell'orlo varia dai $22 \mathrm{~cm}$ ai 26,6. Le tracce di combustione che si osservano sull'esterno delle pareti laterali indicano che si tratti realmente di tipi da fuoco .

31. Pentola con orlo a tesa con superficie superiore piana, parete diritta, carenata. Attacco con la parete sottolineato all'esterno da un sottile listello. La parete inferiore è leggermente arrotondata, il fondo è convesso. Alt.17,4 cm, Dm (orlo): $22 \mathrm{~cm}$, Dm (fondo) $7 \mathrm{~cm}$. Argilla ben cotta, dura al tatto, rosso brunastra (Fig. 13.7, Fig. 25.5).

32. Fr. di orlo e parete di una pentola simile a quella precedente. Il tipo è rinvenibile a Sutri, Cosa, Ostia, Luni e anche a Settefinestre ${ }^{237}$. Dm: 24 cm. Argilla giallo-rossastra, micacea, con tracce verdastre sparse (Fig. 13.,8).

33. Fr. di orlo e parete di una pentola a forma Sutri $20^{238}$. L'orlo a tesa è più spesso della parete laterale leggermente inarcata. Dm: 27,4 cm. Argilla rosso-giallastra, porosa, residui di incrostazione (Fig. 13.9).

34. Fr. di orlo e parete di una pentola a forma Sutri $20^{239} . \mathrm{Dm}: 18,8 \mathrm{~cm}$. Argilla ocra giallastra, porosa.

35. Pentola con orlo leggermente ingrossato verso l'esterno ${ }^{240} \mathrm{Dm}: 25,6 \mathrm{~cm}$. Argilla rosso-giallastra, leggermente porosa (Fig. 13.10).

36. Frammenti dell'orlo e delle pareti di una pentola. L'orlo è meno obliquo, all'interno è leggermente ingrossato. Le pareti sono dritte, con attacco decisamente angolare (Carina) ${ }^{241} \mathrm{Dm}: 24 \mathrm{~cm}$. Argilla giallo-rossastra, porosa, leggermente micacea.

37. Fr. dell'orlo con l'estremità esterna leggermente ingrossata di una pentola tipo Sutri $21 .{ }^{242}$ La parete laterale è leggermente conica. Dm: 26,6 cm. Argilla rosso-giallastra, porosa, leggermente micacea (Munsell 7.5 YR/ 7/6 (Fig. 14.1).

38. Pentola con orlo grosso e listello sottile sul collo. ${ }^{243}$ Argilla brunastra, leggermente micacea, sulla superficie di frattura sedimentazioni di calce (Fig. 14.2).

39. Fr. di pentola con orlo obliquo a tesa. Sotto l'orlo un listello sottile. Dm: $22 \mathrm{~cm}$ Argilla giallo-rossastra (Munsell 7.5 YR 6/6), la superficie di frattura con tracce di calcina (Fig. 14.3).

40. Fr. di pentola. L'orlo è pressoché orizzontale e all'interno si trova un'incisione orizzontale. ${ }^{244}$ Argilla giallo-rossastra, porosa, granulata, micacea (Fig. 14.4).

41. Fr. di pentola con orlo obliquo a tesa e parete laterale conica. Dm: $23,7 \mathrm{~cm}$. Argilla rossa e marrone; la parete laterale è annerita da combustione.

42. Fr. di pentola. Orlo a tesa obliquo. Dm: $22,8 \mathrm{~cm}$. Argilla rosso-giallastra, porosa.

43. Fr. di pentola. Con orlo a tesa ingrossato, obliquo. Dm: $25,3 \mathrm{~cm}$. Argilla marrone-giallastra, annerita da combustione all'esterno.

44. Fr. di parete, la carena è accentuata. Argilla marrone-giallastra (Munsell 7.5 YR 5/6, annerita da combustione sia all'interno, sia all'esterno (Fig. 14.5).

45. Fr. di pentola. Con orlo a tesa obliquo che tende ad assottigliarsi. Pareti laterali coniche. Dm: $24,9 \mathrm{~cm}$. Argilla rosso-giallastra, con macchie brunastre (Fig. 14.6)? Fig. 25,8.

46. Fr. di pentola con orlo piuttosto corto che va leggermente assottigliandosi ${ }^{245}$. Dm: $23,2 \mathrm{~cm}$. Argilla color mattone, ben cotta, dura al tatto.

47. Fr. di pentola con orlo estroverso obliquo e pareti laterali leggermente coniche. Dm: $23,9 \mathrm{~cm}$. Argilla rosso-giallastra.

48. Tre fr. di pentola profonda, a profilo a forma di S, con orlo inclinato verso l'esterno con superficie interna a profilo semicircolare. Parete a profilo S. Dm: 21,4 cm. Argilla rossa e gialla, porosa. ${ }^{246}$ (Fig. 14.7). Probabilmente serviva per la cottura o per mescolare gli ingredienti ${ }^{247}$.

49. Pentola a profilo a forma di S, simile alla precedente, sulla spalla un listello leggermente sporgente. Dm: 10,6 cm, Argilla giallo-rossastra, porosa.(Fig. 14.8)

${ }^{236}$ OLCESE 2003, 76.

${ }^{237}$ Sutri forma 21, DunCAN 1964, fig. 10,71; Dyson 1976, p.117, fig.43, tipo 22, fig.44,3; OstiA XIII. fig.44,87; LUNI II. Tav.270,3; Settefinestre Tav.24,13.

${ }^{238}$ DunCAN 1964, fig. 10,70.

${ }^{239}$ DunCan 1964, fig. 10,69.

${ }^{240}$ LuNi II. Tav.270,6.

${ }^{241}$ Dyson 1976, fig.43,10 22 II.

${ }^{242}$ DUNCAN 1964, fig. 10,71.

${ }^{243}$ DysON 1976, fig.44,15 22 II; LUNI II.tav.270, 6.
${ }^{244}$ Dyson 1976, fig.44,16 22 II.

245 Settefinestre tav.27,13.

246 Duncan 1964, fig. 3,50; Vegas 1973, pp.34-35, fig.7; LuNI II. p.507, tav.263, fig.14; DYSON 1976, 102, fig.37 tipo PD 110 Settefinestre Tav.54,2.

${ }^{247}$ Ch. Bonnet-C. Batigne Vallet: Contribution à l'étude des céramiques grises kaolinitiques Voconces d'après le mobilier issu des fouilles du tracé drômois du TVG mediterranée. Acta RCRF 37 (2001) 81. 

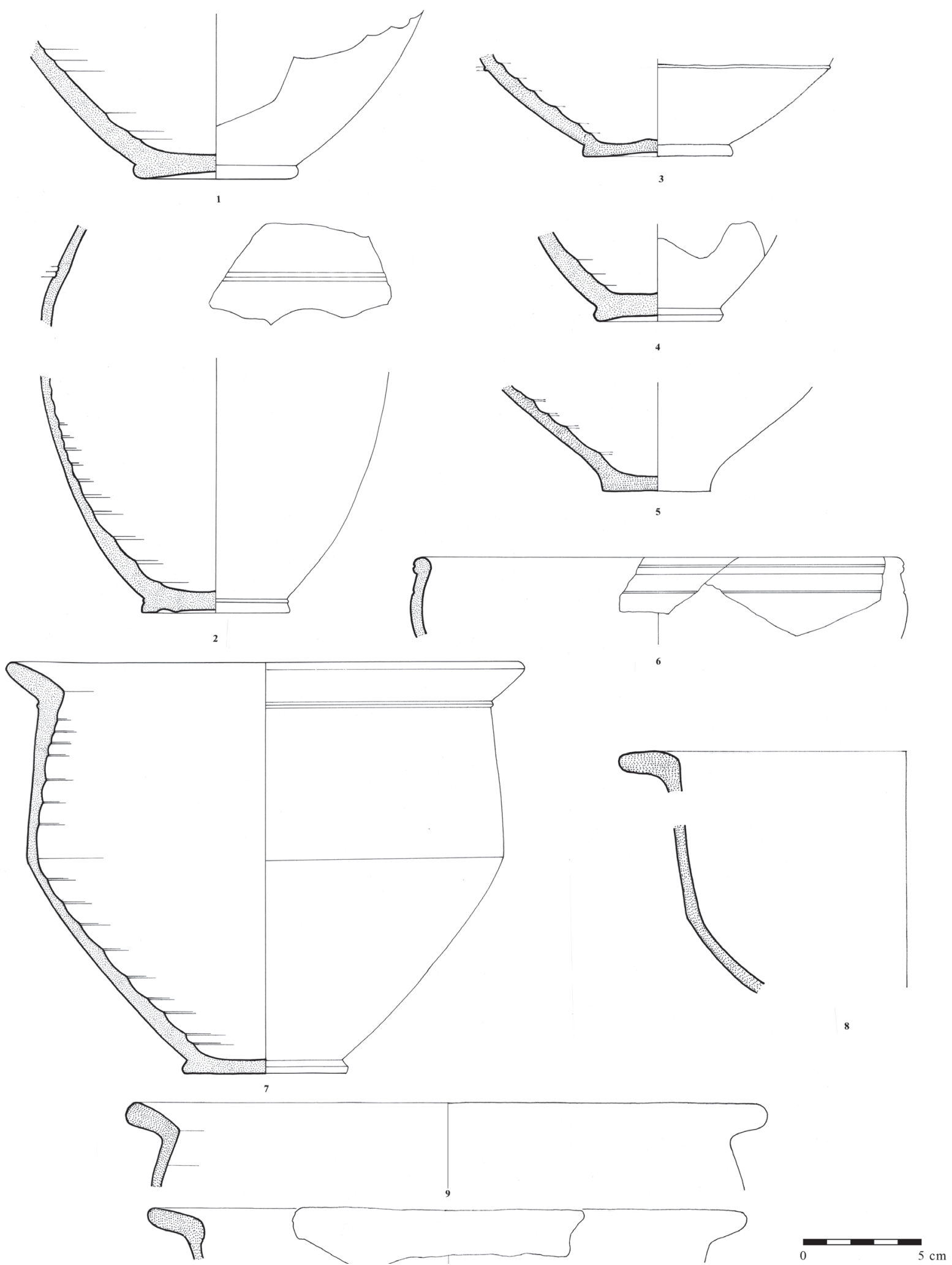

Fig. 13. Brocche, coppa e pentole nel canale conducente alla cisterna del centro del cortile settentrionale. 


\section{Pentole carenate con orlo obliquo a tesa}

Questa tipologia sia nella forma sia nell'esecuzione tecnica imita le pentole nord-africane. A Luni è stato rinvenuto in contesti provenienti dal periodo che va dalla metà del III sec. al sec. $\mathrm{V}^{248}$, mentre altrove, in un piccolo insediamento situato tra Pisa e Lucca è databile al III sec. Su uno dei fr. che abbiamo rinvenuto strisce verticali di rivestimento rosso dai contorni irregolari.

50. Parte superiore di una pentola, con orlo obliquo a tesa, con faccia superiore leggermente convessa. Parete leggermente inclinata verso l'interno. La carena è accentuata. Sulla parte superiore tracce di rivestimento rosso anche nella parete interna. La pentola è ricomposta da 8 frammenti. ${ }^{249} \mathrm{Dm}: 18,6 \mathrm{~cm}$, Argilla rosso-giallastra, porosa (Fig. 11.2).

51. Fr. ricomposto di due pezzi, simile al precedente, con sopra la carena un'incisione, mentre sotto l'orlo troviamo due listelli molto sottili. Dm: $19 \mathrm{~cm}$. Argilla giallo-rossastra, leggermente porosa (Fig. 14.9).

52. Fr. di una pentola di forma Sutri 51, con orlo corto, obliquo, inclinato verso l'esterno ${ }^{250}$ Argilla giallo-ocra, leggermente porosa.

53. Fr. di pentola un po' più bassa delle precedenti con orlo a tesa quasi orizzontale. La carena è poco accentuata, il fondo è leggermente arcuato. ${ }^{251} \mathrm{Dm}: 19,6 \mathrm{~cm}$. Argilla rosso-giallastra.

54. Fr. di pentola simile alla precedente. ${ }^{252}$. Dm: $19,5 \mathrm{~cm}$. Argilla ocra, poco porosa.

55. Tre fr. di pentole simili alle precedenti con carena molto accentuata. ${ }^{253} \mathrm{Dm}: 19 \mathrm{~cm}$. Argilla rosso-giallastra, porosa (Fig. 14.10)

56. Fr. di orlo di pentola. La superficie superiore dell'orlo piuttosto grosso è concava, pareti dritte. ${ }^{254} \mathrm{Dm}: 11,4 \mathrm{~cm}$. Argilla giallo-rossastra, porosa (Fig. 14.11).

\section{Olle}

La proporzione ridotta di rinvenimento di questo tipo di vasi del resto frequente nel materiale del canale che porta alla cisterna del cortile $\mathrm{N}$ è sorprendente, e gli esemplari venuti alla luce in questo sito sono anche di forma differente rispetto a quella delle tipologie abituali della Campania. Tre sono di forma 26 , con collo relativamente lungo e dritto, imboccatura tendente leggermente a ingrossarsi e avente una leggera incisione.

57. Olla con orlo obliquo, sagomato, pareti bombate. ${ }^{255} \mathrm{Dm}: 17,7 \mathrm{~cm}$. Argilla:rosso-giallastra, porosa. (Fig. 15.1)

58. Parete di un'olla di forma Sutri 32. ${ }^{256}$ Argilla color ocra, leggermente porosa, annerita da combustione (Fig. 15.2).

59. Fr. di un'olla con orlo obliquo, sagomato. Dm: $16 \mathrm{~cm}$. Argilla rosso-giallastra, porosa.

L'altra forma è caratterizzata dal collo corto, e dall'orlo leggermente ingrossato e arrotondato.

60. Olla con collo breve, orlo con superficie esterna concava. ${ }^{257} \mathrm{Dm}: 15,4 \mathrm{~cm}$. Argilla rosso-giallastra, porosa (Fig. 15.3 ). Il confronto rinvenuto a Settefinestre è databile all'età tardo antonina.

\section{Tegami}

Alla forma equivalente al tipo Olcese 7 possono essere inquadrati due vasi ricorrenti raramente a San Potito.

61. Tegame con orlo a mandorla, parete arrotondata, fondo concavo, piede ad anello. ${ }^{258} \mathrm{Dm}: 23,6 \mathrm{~cm}$. con rivestimento rosso all'esterno ed all'interno, Argilla giallo ocra, leggermente porosa (Fig. 15.4).

${ }^{248}$ S. MENCHELLI: Vasi comuni nella Tuscia settentrionale costiera. Aspetti regionali ed apporti mediterranei. Acta RCRF39 (2005) 113, tipo 12.

${ }^{249}$ Dyson 1976, LS 12; Settefinestre Tav.24,13 ANNESE 2000, tipo 5.1; ŽERJAL 2008, fig.4,13.

${ }^{250}$ DunCAN 1964, fig. 16,186.

${ }^{251}$ H.S. Robinson: Pottery of the Roman Period. Chronology. The Athenian Agora V. Princeton NJ. 1959, G 193, 155-6, K 92; DYSON 1976, fig.43,12 22 II; T. ŽERJAL: Eastern imports in the ager Tergestinus. Acta RCRF 40 (2008) fig.4,13. Knossos type 2- J.W. HAYES: The Villa Dionysos Excavations, Knossos The Pottery. Annual British School Athens 78( 1983) 106.
${ }^{252}$ LunI I. tav. 60, 6 CM 1095/2; DysON 1976, fig.43,13; Carbonara-Messineo 1991, 248

${ }^{253}$ Luni I. Tav.6 7CM, 1230/6; Settefinestre Tav.25,3.

${ }^{254}$ LUNI I. tav.72 12 CM 2122/1; LUNI II. tav.273,6; DYSON 1976, fig.44,15; MeNCHELLi 2005, tipo 16.

${ }^{255}$ DUNCAN 1964, fig. 11,100 - a però la sagomatura dell'orlo è differente; DYSON 1976, fig.47,53; QuILICI 1976. fig.43,448 (Castel Giubileo); LUNI II, tav.272,2. Evoca piuttosto la forma 2311 b di Pompei (Di Giovanni 1996, fig. 19).

${ }^{256}$ DunCAN 1964, fig. 13, 127.

${ }^{257}$ Dyson 1976, 56, fig.16 - tipo 16; Luni II. tav.272,2; Settefinestre, tav.30,3.

${ }^{258}$ LuNi II, tav. 269,14; MenCHELli 2012, fig.3,21. 

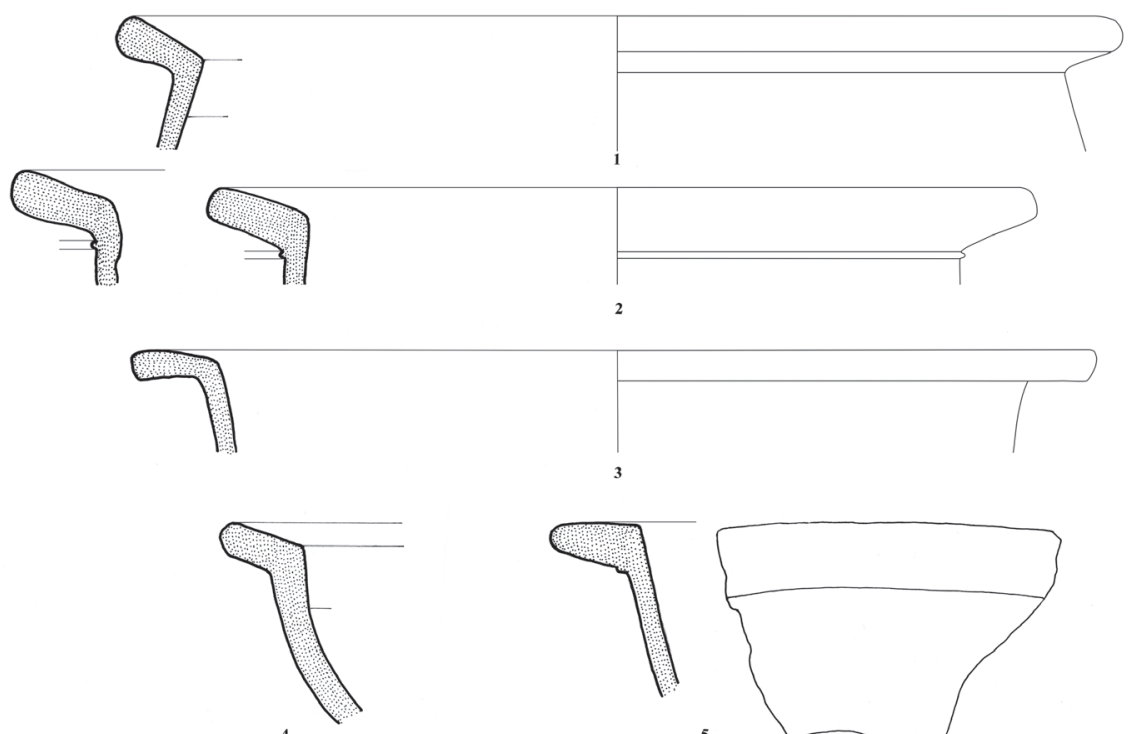

4
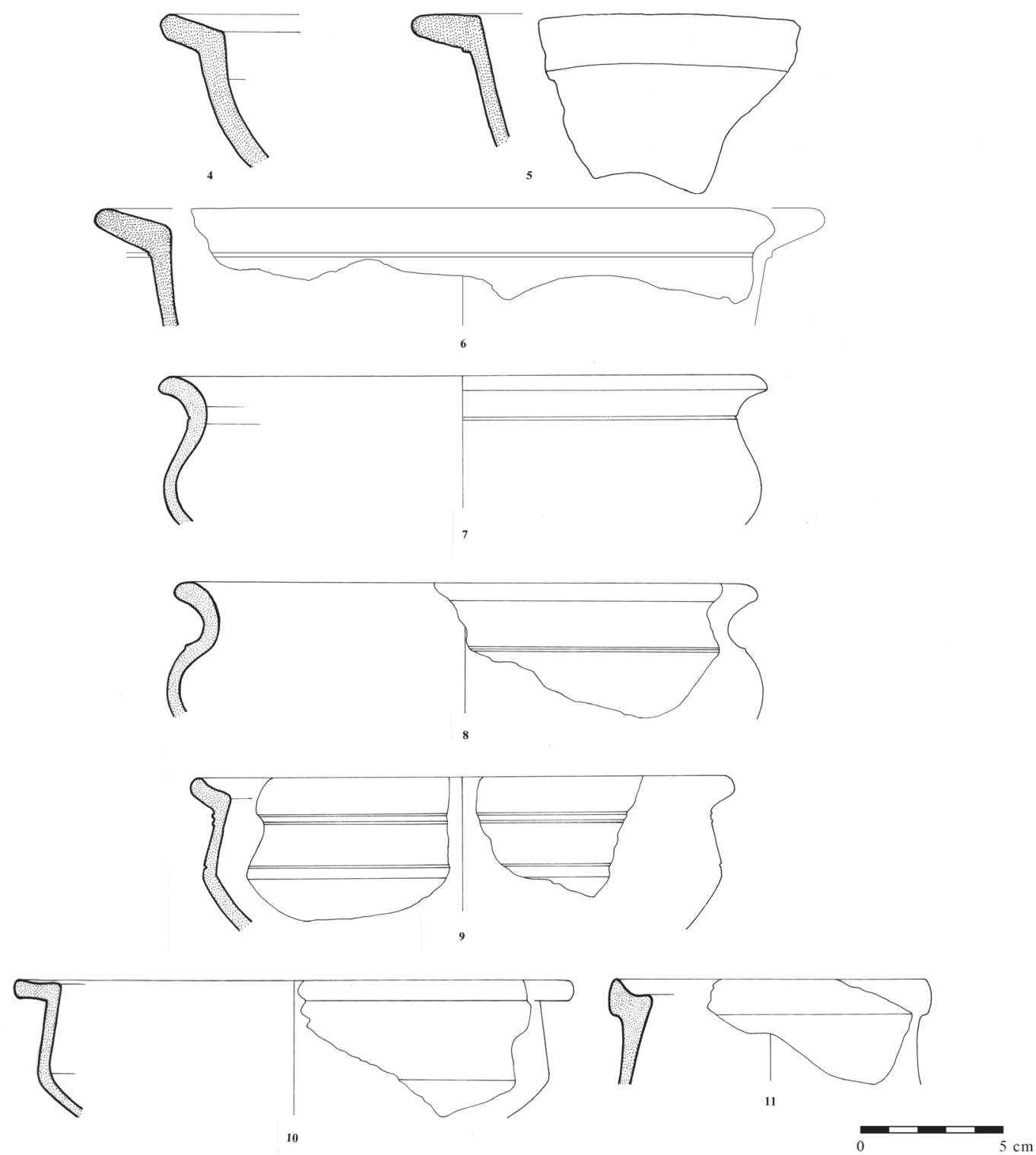

Fig. 14. Pentole con orlo a tesa rinvenuti nel canale conducente alla cisterna nel centro del cortile settentrionale. 

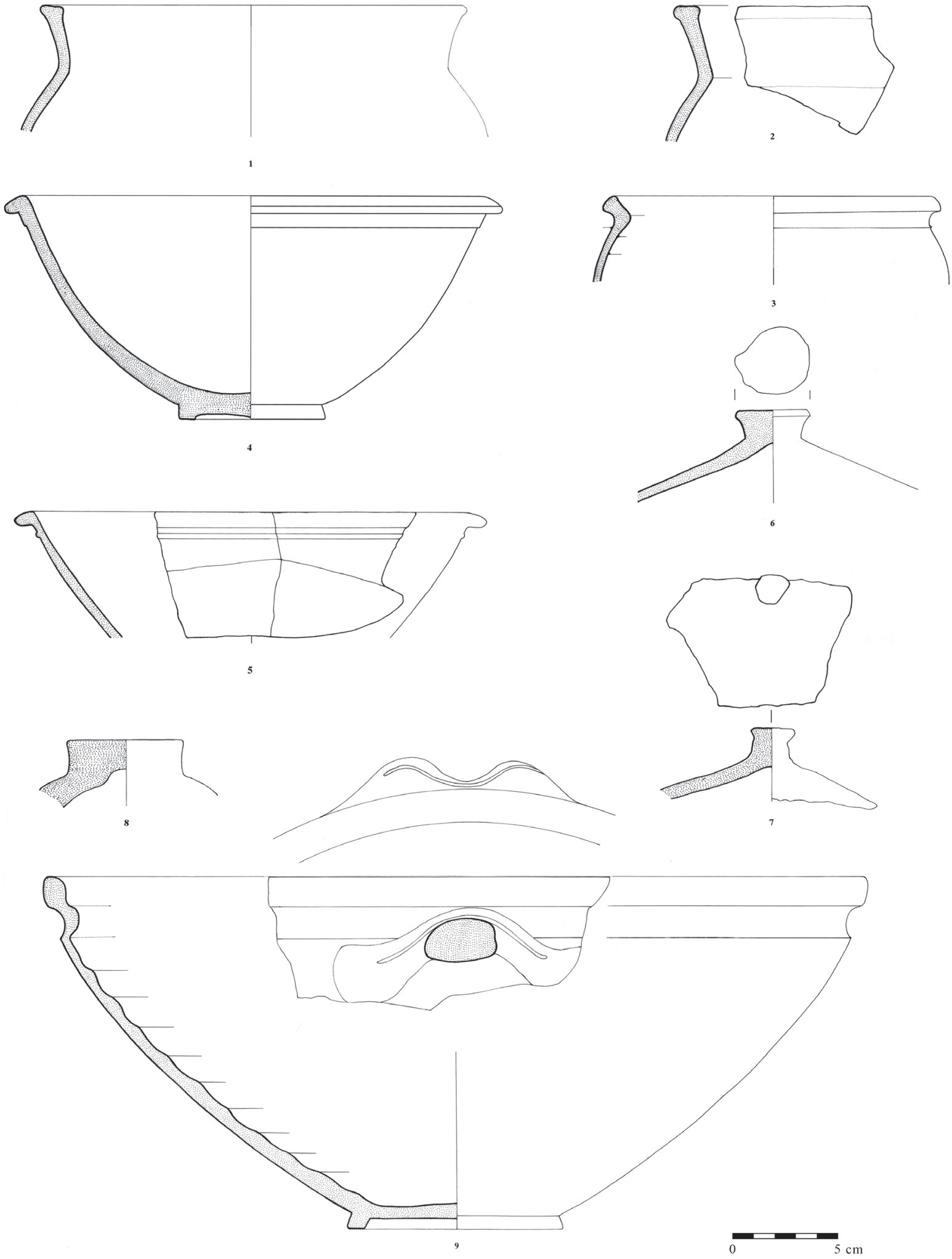

Fig. 15. Olle, coperchi e bacini rinvenuti nel canale conducente alla cisterna nel centro del cortile settentrionale. 
62. Tegame ricomposto di 6 frammenti con orlo estroflesso, parete svasata che si restringe verso il basso, orlo a mandorla. ${ }^{259}$ Sotto 1'orlo uno stretto listello orizzontale. Dm: $22,6 \mathrm{~cm}$., con rivestimento macchiato e molto screpolato; argilla ocra giallastra, poco porosa (Fig. 15.5).

Si conoscono confronti di questa forma derivanti da Bolsena, Cosa, La Celsa sita nella città di Roma, Sutri e Vasanello. ${ }^{260}$ Gli esemplari equivalenti al tipo sono databili al I sec. ${ }^{261}$ ma S. Menchelli menziona confronti derivanti dal periodo che va dal sec. II fino l'età tardo romana ${ }^{262}$ dell'esemplare recuperato per primo.

\section{Coperchi}

63. Coperchio troncoconico con manico pure troncoconico. ${ }^{263}$ Argilla rossa e ocra, porosa (Fig. 15.6).

64. Fr. di un coperchio a profilo arrotondato, con orlo a mandorla ${ }^{264}$ tipo Olcese 4 . Dm: di cca. $21 \mathrm{~cm}$. Argilla rosso-giallastra, porosa. Confronti derivanti dal periodo tra l'età claudia e tardo-severina. (Fig. 15.7) 265

65. Coperchio troncoconico? Con orlo piatto, all'interno leggera solcatura. ${ }^{266}$. Argilla rosso-giallastra, porosa (Fig. 15.8).

\section{III c Ceramica per la preparazione}

\section{Bacini}

Nel riempimento del canale che porta alla cisterna sono stati rinvenuti diversi frammenti di una tipologia rara, un bacino biansato di grandi dimensioni. Ultimamente fu F. Fabbri ad occuparsi di questo tipo, facendo derivare la forma da tradizioni ellenistiche; lei aveva trovato a Corinto e Apollonia forme simili e volle presentarne i confronti italici. ${ }^{267}$ In Italia lei ne identificò 7 varianti facendo notare che, questi vasi quasi dappertutto possono essere ritenuti di produzione locale. In Etruria oltre a Vigoni la tipologia fu fabbricata anche a Torrita di Siena, questa forma fu rinvenuta nella fornace di Umbricius Cordus. ${ }^{268}$ Gli esemplari di Segesta, località dell'Italia Meridionale ${ }^{269}$, e quelli venuti alla luce a $\operatorname{Cosa}^{270}$ vengono ritenuti imitazioni locali di merce importata. G. Olcese pubblica alcuni confronti derivanti dalla Campania ${ }^{271}$, Vasanello, Gabii ${ }^{272}$ e Ostia. ${ }^{273}$ La tipologia di vaso è collegabile originariamente a un culto. A Corinto fu impiegata come thymaterion, mentre in Etruria serviva a scopi sepolcrali. ${ }^{274}$ A Pompei, Segesta e Pistoia, dove si poté datare questa tipologia, venne alla luce in contesti di alta età, dal II sec. al I sec. a. C.; a Cosa fu rinvenuta in contesti dell'età di Augusto e di Claudio. Il riempimento del canale di San Potito può essere datata al III sec., ma nemmeno il canale stesso può derivare da un periodo precedente alla seconda metà del II sec. Per colmare il cortile settentrionale, quindi, furono impiegati scarti contenenti frammenti di vasi derivanti da periodi di prima.

66. Bacino a vasca profonda con orlo estroflesso e rialzato, con profonda scanalatura interna, vasca emisferica distinta e carenata nella parte superiore, con due anse a bastoncello addossate alla parete. ${ }^{275} \mathrm{Dm}: 39,4 \mathrm{~cm}$. Argilla dura, color marrone medio e scuro (Munsell 2.5 YR da 5/4 e 5/8) con mica (Fig. 15.9, Fig. 25.9).

${ }^{259}$ OlCESE 2003, tav.XVI.3; S. Menchelli: Per una classificazione delle ceramiche comuni di età romana nell'Etruria settentrionale costiera. Acta RCRF 38 (2003) 115, fig.3,3.

${ }^{260}$ La Celsa 4 forma, Sutri 52 forma.

${ }^{261}$ OlCESE 2003, 87.

${ }^{262}$ Menchelli 2012, fig.3,21.

${ }^{263}$ Vegas 1968, Abb. 16,160.

${ }^{264}$ DYSON 1976, LS 55; OlCESE 2003, tav.XX.7.

265 Olcese 2003, tav.XX.7, 254, 256.

${ }^{266}$ CARBONARA-MESSINEO 1991, fig. 247,4.

${ }^{267}$ F. FABBRI: Ceramic basins from the "kilns of Vigoni" in Scandici (Florence) Italy. Aegean shapes in pottery production in the area of Roman Florentia. Acta RCRF 40 (2008) 341-344.
${ }^{268}$ G. PUCCI: L'officina di un ceramista romanoe. Il territorio di Torrita di Siena nell'antichità. Firenze 1992, 130 n. 120.

${ }^{269}$ FABBRI 2008, 342.

${ }^{270}$ DYSON 1976, 127 n, 78, 22II-78.

${ }^{271}$ OlCESE 2003, tav.VI.2, tipo 1.

272 Vegas 1968, Abb. 17,173.

${ }^{273}$ G. Gasparetti: Produzione e consumo della ceramica comune da mensa e dispensa. In: Les céramiques communes de Campanie et de Narbonnaide (I er s. av.J.-C-II e s. ap.J.-C.). La vaiselle de cusine et de table. Actes des Journées d'étude organnisées par le Centre Jean Bérard et la Soprintendenza Archeologica per le province di Napoli e Caserta (sous la direction M. Bets) Naples 1996 25, fig. 1,9.

${ }^{274}$ FABBRI 2008, loc.cit.

${ }^{275}$ FABBRI 2008,344, fig.5,3. 
67. Fr. di un bacino con orlo inclinato verso l'esterno con superficie esterna convessa. Parete inclinata verso l'interno, forma Sutri $62 .{ }^{276}$ Vaso con pareti spesse, leggermente emisferico. Dm: $36 \mathrm{~cm}$. Argilla ocra giallastra con tono tendente al bruno, leggermente porosa (Fig. 16.1).

Quest'ultimo esemplare fu reperito dalla colmatura della tettoia della cisterna.

68. Fr. di un bacino con orlo a tesa. La parte esterna dell'orlo tende a ingrossarsi. $\mathrm{Dm}: 30,3 \mathrm{~cm}$. Argilla giallo-rossastra, con inclusioni bianche. ben cotta, dura al tatto (Fig. 16.2)

69. Fr. di un bacino con orlo inclinato verso l'esterno, a profilo triangolare. Parete a profilo arrotondato. ${ }^{277}$ Argilla giallo-rossastra, porosa (Fig. 16.3).

70. Fr. simile al precedente. Argilla giallo-rossastra,con inclusioni di calce. Il prototipo di questa forma dovette essere la merce pontina databile alla fine del I sec. e all'inizio del II. (Fig. 16.4) ${ }^{278}$

\section{III d Lucerne}

Ansa di una lucerna di forma Loeschcke VIII=Denavue VII=Bailey O-P=Leibundgut XX-XXIII. Argilla ocra giallastra, molto porosa (Fig. 16.5).

Si conoscono numerose varianti di questa forma che, è largamente diffusa in tutto l'Impero Romano. La sua produzione ebbe inizio nell'età tiberiana, avendo poi ampia diffusione per tutto il II sec. Il nostro esemplare può essere datato al periodo che va dall'età tardo flavia fino all'età antonina.

\section{III f Anfore}

Parte dell'orlo ingrossato di un'anfora di forma Dressel 2-4. Argilla giallo-rossastra (Fig. 16.6).

Questo tipo ricorre spesso in quantità notevole anche nel materiale pubblicato precedentemente. ${ }^{279}$ Probabilmente si tratta di un esemplare derivante da un periodo tra la fine del I sec. e il II.

Confrontando i reperti portati alla luce dai due pozzi, si osserva la differenza che corre tra gli spettri degli stessi. Pur essendo in ambedue i contesti la ceramica da fuoco presente in maggior quantità rispetto alla ceramica da mensa tuttavia poi, entro questo ambito si osservano discrepanze. Nel contesto di reperti del pozzo di prima epoca (taglio g-4) la quantità delle olle e delle pentole è più considerevole, mentre nel contesto della cisterna scavata al centro del cortile settentrionale accanto alle pentole ed i tegami anche la proporzione dei bacini è ragguardevole.

In base alla relativa cronologia pare che la cisterna settentrionale e particolarmente il canale che vi conduce sono molto più tardi rispetto al pozzo scavato nel taglio g-4, su cui nel periodo di costruzione seguente fu costruito l'edificio a doppie pareti. Pur tuttavia la cronologia della ceramica dei due contesti rivela pochissime differenze; la quantità della ceramica databile alla seconda metà del II sec. o al III sec. è di gran lunga minore rispetto alla quantità presumibile, ma spesso perfino la cronologia di questi tipi “tardi” è discutibile. Per es. la quantità della ceramica a pareti sottili è maggiore nella cisterna $\mathrm{N}$ rispetto a quello $\mathrm{S}$ (la proporzione è di 19:14), ma è anche vero che, in quello settentrionale compariscono anche tipologie derivanti dalla fine del II sec. (Kat. 22, 24). Nel materiale rinvenuto nella cisterna $\mathrm{N}$ le pentole che imitano le forme nord africane, in più alcuni tipi di tegami, possono essere datati a un periodo più tardo (seconda metà del II sec. eventualmente perfino il III), gli altri tipi o sono databili ad un periodo precedente o non sono affatto databili. È indubbio che il pozzo del taglio g-4 databile in base alla moneta coniata nel 101/102 probabilmente fu, colmato all'inizio del II sec., mentre la cisterna settentrionale e il canale scavato più tardi del primo furono colmati solo nel corso del III sec. Si ipotizza quindi, che durante il riempimento di questa parte del cortile settentrionale nel materiale scavato dovettero versare un deposito anteriore, attestato, tra l'altro, anche dalla quantità della ceramica a pareti fini.

\footnotetext{
${ }^{276}$ DunCAN 1964, fig. 18,213; Dyson 1976, fig.47,46 22II; Settefinestre taf.53,6.

${ }^{277}$ Settefinestre Tav.53,3; Ostia III. 362, tav.XLV, fig.163.
}

${ }^{278}$ D. REYNOLDS-D.R.HERNANDEZ- D.ÇONDI: Excavations in the Roman Forum of Buthrotum (Butrint) first to third century pottery assamblages and Trade. Acta RCRF 40 (2008) fig.7,14.

${ }^{279}$ HÁRSHEGYI 2008, 135-136. 


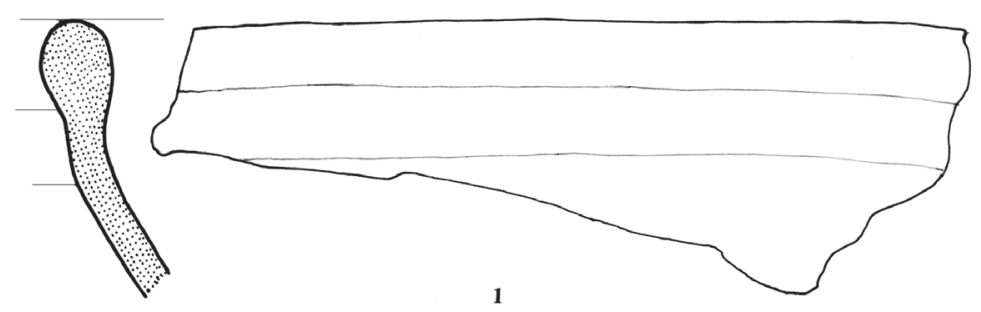

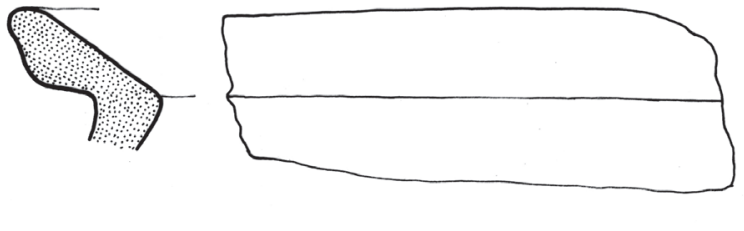

2

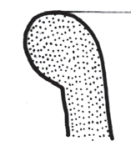

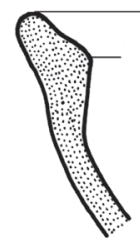
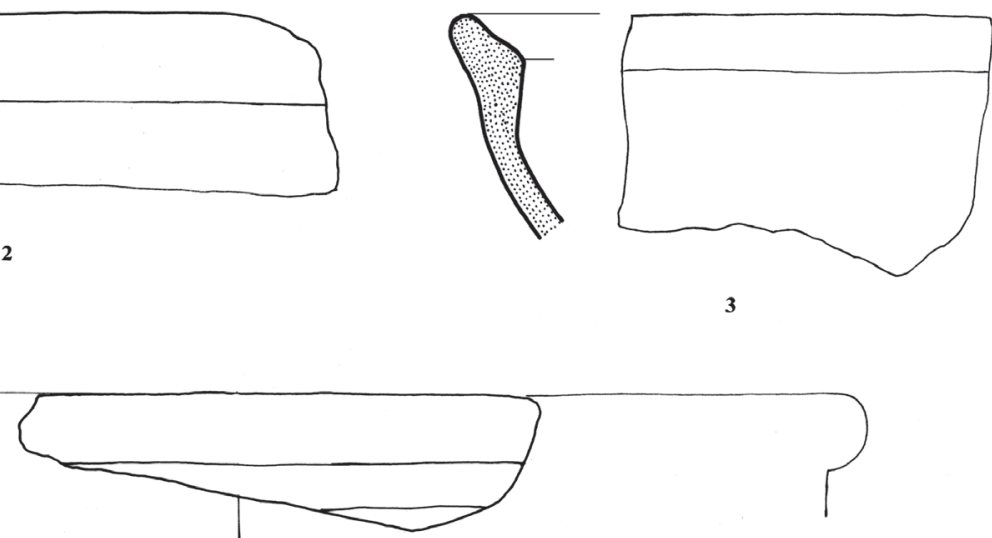

4
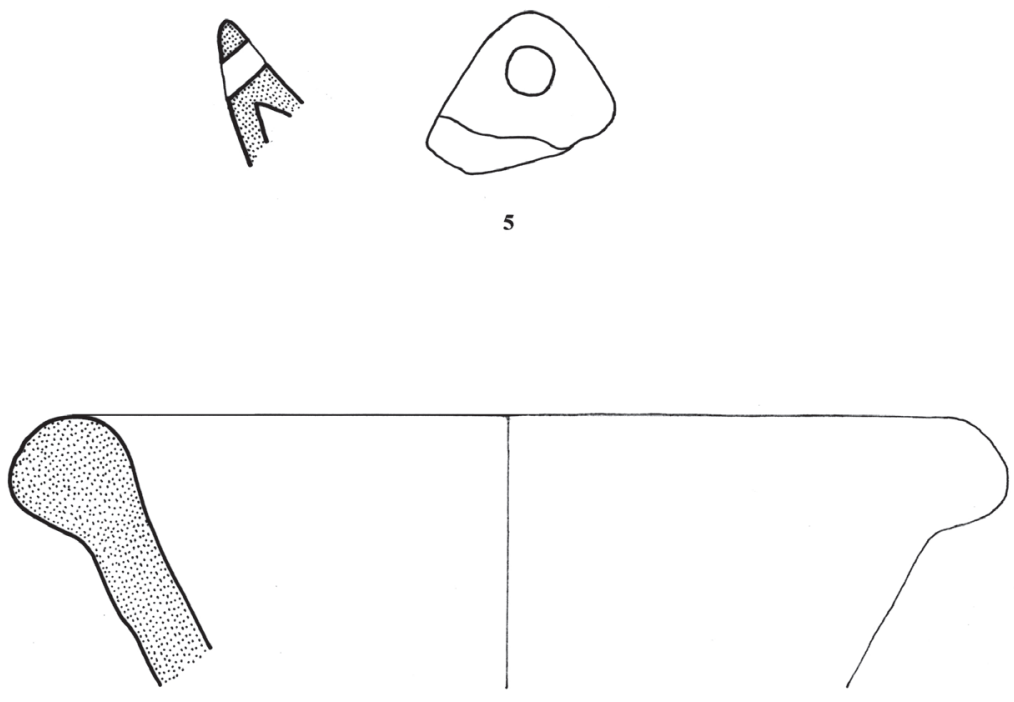

6

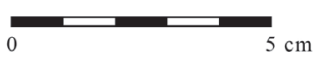

Fig. 16. Bacini, lucerna e anfora rinvenuti nel canale conducente alla cisterna nel centro del cortile settentrionale.

Acta Archaeologica Academiae Scientiarum Hungaricae 66, 2015 


\section{CONTESTO DI REPERTI RINVENUTI NELLO STRATO D'INCENDIO SCAVATO NELLA PARTE S DEL I EDIFICIO.}

Mel corso dei nostri lavori di scavo eseguiti negli anni 2008 - 2009 nel taglio c-4 abbiamo rinvenuto uno strato "chiuso" nell' angolo SE della villa di prima costruzione, situato a Ovest da un vano dotato di abside e probabilmente mai preso in uso (Fig. 17). Nel ambiente con abside di grandi dimensioni non abbiamo trovato il livello di calpestio e nemmeno relativi reperti. Pare probabile che il locale non fosse mai preso in uso per via di un terremoto sopravvenuto durante i lavori di costruzione o pochissimo dopo. Nello stesso tempo nella specie di piccolo corridoio attiguo, sotto uno strato di scarti contenenti malta e calce, a $77 \mathrm{~cm}$ di profondità dalla cima del muro perimetrale, si è potuto osservare uno strato d'incendio, il cui fondo fu raggiunto a $116 \mathrm{~cm}$ di profondità. Nello strato d'incendio vennero alla luce diverse tegole, malta, frammenti di intonaco dipinto. Nello strato d'incendio contenente carbonella si vedevano delle strisce di cenere sparse, e altrove abbiamo trovato zolle di argilla rossastre e bruciate. La superficie bruciata avente un fondo rosso, comparisce anche sotto lo zoccolo dell'edificio con muri doppi, di costruzione più tarda. Questo è lo strato in cui abbiamo rinvenuto l'unica sigillata derivante da La Graufesenque, di forma Drag. 29, ${ }^{280}$ diversi frammenti di ceramica, tasselli di mosaico di vetro blu e blocchi di pasta vitrea di color blu che probabilmente servivano per ritagliarne i tasselli di mosaico. La maggior parte di questi pezzi non aveva una forma regolare, ma erano simili a schegge o erano più grandi di un tassello normale. Tutto ciò fa pensare che si tratti del materiale di un'officina o di un magazzino, dove questo mosaico fu prodotto o usato e i pezzi di scarto fossero raccolti qui. Tutti i reperti si trovano sopra un pavimento di argilla bruciato in rosso, il quale è nel livello del basamento delle costruzioni a forma di U. Nello stesso strato vennero alla luce anche pezzi di vetro da finestra e di marmo e pochi ossi di uccelli. Il fondo dello strato d'incendio era indicato da un piano rossastro.

Nel vano abbiamo osservato due piani di calpestio, il superiore era coperto da uno strato di detriti misti a carbonella, in cui si rilevano frammenti delle travi bruciate e delle tegole cadute dal tetto. Sotto lo strato annerito da combustione, abbiamo scavato tasselli di pasta vitrea, gusci di conchiglie, pochi ossi animali, frammenti di ceramica e un fr. di una lucerna di forma Dressel 4, tipo Vogelkopflampe. Il livello sottostante, costituito da mattoni e calcina, finiva a $15 \mathrm{~cm}$ sopra il basamento del muro di $\mathrm{S}$ e sotto si estendeva uno strato di terra vergine gialla argillosa.

Abbiamo rinvenuto nello strato d'incendio in tutto 132 frammenti di ceramica. Il complesso di reperti conteneva 2 terra sigillate, 4 ceramiche a pareti sottili, 9 anfore, 2 lucerne e numerosi tasselli di mosaico di pasta vitrea blu.

\section{IV a Ceramica da mensa}

\section{Brocche}

1. Ansa a nastro con due solcature. Larghezza dell'ansa: $4,1 \mathrm{~cm}$. Argilla ocra giallastra, leggermente porosa (Fig. 18.1). (gruppo I)

2. Ansa a nastro con due solcature leggermente incise. Larghezza dell'ansa: $5 \mathrm{~cm}$. Argilla ocra giallastra, leggermente porosa (Fig. 18.2).

3. Sette fr. di brocca con ansa a nastro a due solcature leggermente incise. Larghezza dell'ansa:2 cm. Argilla giallo-rossastra, porosa con molte inclusioni di calce. (gruppo II)

4. Ansa a nastro con sezione quasi ovale. Larghezza dell'ansa:1,7 cm. Argilla ocra giallastra, leggermente porosa (Fig. 18.3).

\section{Tazze}

5. Tre fr. di tazza leggermente ovoidale. Argilla ocra giallastra. leggermente porosa. (gruppo IV)

6. Piccolo fr. di orlo a goccia. Argilla giallo-rossastra (Fig. 18.4).

\section{Coppe}

7. Fr. di una coppa con orlo distinto. Argilla ocra giallastra, leggermente porosa (Fig. 18.5).

8. Fr. dell'orlo di una coppa con imboccatura a sezione a forma di goccia.. Dm: $20 \mathrm{~cm}$. Argilla giallo-rossastra, porosa. (gruppo I a)

${ }^{280}$ D. GABLER: Sigillate galliche meridionali ad Emona e in Italia. In: Emona med Akvilejo in Panonijo-Emona between Aquileia and Pannonia. (ed. I. LAZAR-B. ŽupaneK). KoPER 2012, 52, 54-55. 

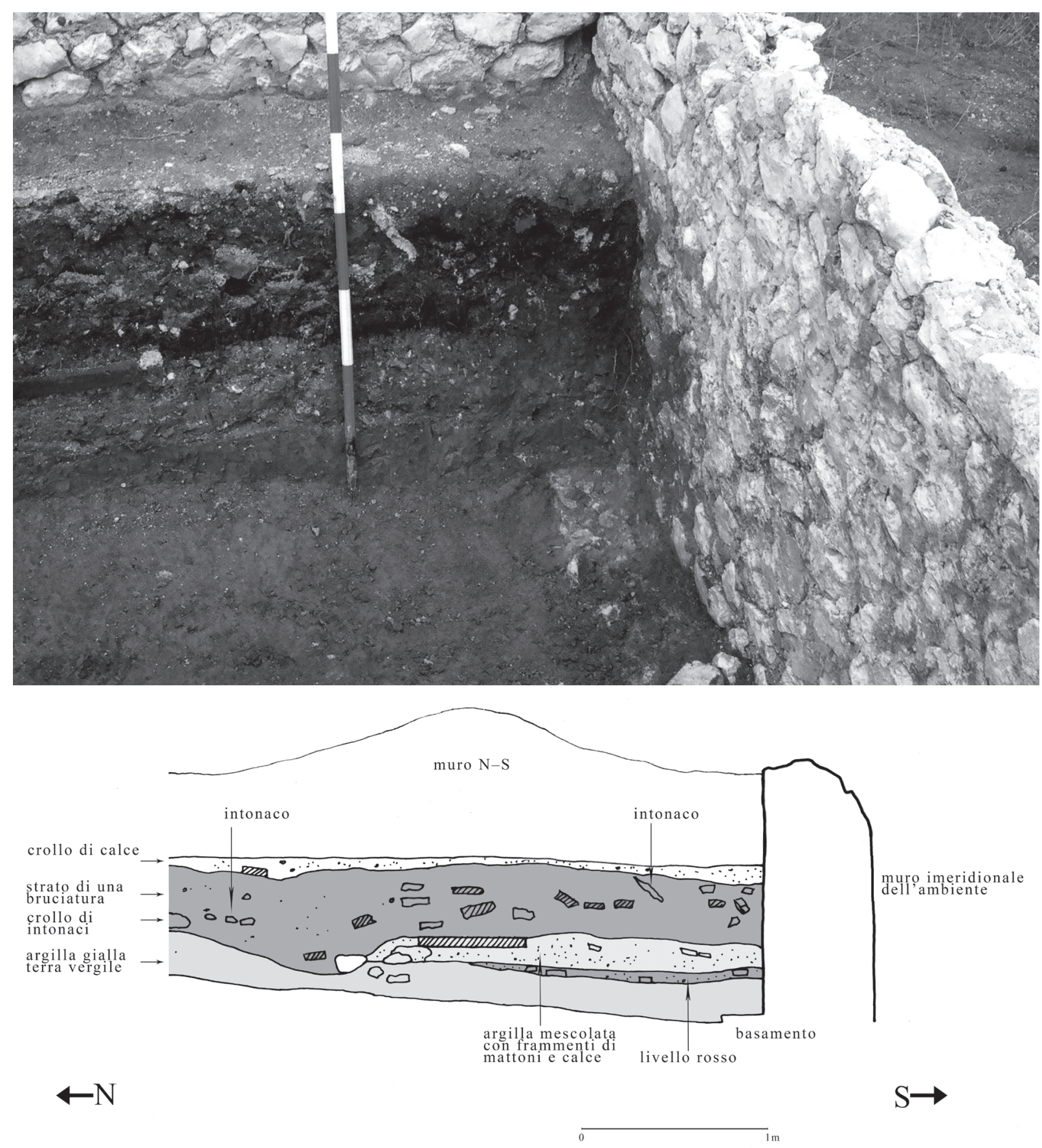

San Potito sezione nel "corridoio"

Fig. 17. Sezione dello strato d'incendio sito nella parte meridionale della villa più antica. 


\section{IV b Ceramica da fuoco}

\section{Pentole}

La lunga cronologia di questo tipo va dalla prima metà del I sec. ${ }^{281}$ fino all'epoca tardo romana. ${ }^{282}$ È inquadrabile al tipo denominato Di Giovanni 2210,6.

9. Pentola ricomposta da 7 frammenti, con orlo a tesa leggermente inclinato verso l'interno. Parete inferiore a profilo arrotondato con un'incisione orizzontale. ${ }^{283} \mathrm{Dm}: 26 \mathrm{~cm}$. Argilla ocra giallastra, con inclusioni di calce, ben cotta, dura al tatto (Fig. 18.0).

10. Orlo di una pentola con orlo a tesa, inclinato verso l'interno con una solcatura. Argilla giallo-rossastra, porosa (Fig. 18.7).

\section{Scodelle}

11. Orlo distinto di una vasca troncoconica ${ }^{284}$, con un listello sull'orlo. Dm: $27,4 \mathrm{~cm}$, Argilla ocra giallastra, porosa, cotta all'interno di color grigio (Fig.18,8).

La tipologia ebbe ampia diffusione nel periodo tra l'età flavia e l'epoca tardo-romana. ${ }^{285}$

\section{Olle}

12. Parte superiore di un'olla con orlo distinto (sezione a goccia), breve collo troncoconico con corpo ovoidale, marcato con listello spigoloso. Dm:7,8 cm. Argilla ocra giallastra, all'interno inclusioni di calce. A Ostia questa tipologia appare nello strato V B (Fig. 18.9). ${ }^{286}$ (gruppo I a)

13. Fr. di orlo e collo di olla simile alla precedente. Dm: $12,3 \mathrm{~cm}$. Argilla ocra giallastra, digrassata con piccole pietruzze (Fig. 18.10).

14. 12 fr. della parte superiore di un'olla biansata, simile alla precedente con orlo ingrossato, ansa a nastro impostata sotto un listello spigoloso. ${ }^{287}$ Fondo piano. Dm: $12,7 \mathrm{~cm}$, fondo: $7,8 \mathrm{~cm}$, Argilla ocra giallastra, poco porosa, mal cotta, con inclusioni di calce (Fig. 19.1) (gruppo I a).

15. Olla con corpo globulare e fondo piano. L'olla può essere monoansata. ${ }^{288} \mathrm{Dm}$ (fondo) 6 cm, Argilla ocra brunastra, porosa (Fig. 19.2) (gruppo I b).

16. Frammenti delle pareti di un'olla. La superficie è accidentata. Argilla ocra giallastra, poco porosa. (gruppo I a )

17. Fondo di un'olla ricomposto da 4 frammenti. ${ }^{289} \mathrm{Dm}: 7 \mathrm{~cm}$, Argilla brunastra all'esterno (Fig. 19.3).

18. Fondo e parete di un'olla ricomposto da 6 frammenti. Argilla marrone rossastra, all'interno grigia. (gruppo I a )

19. Orlo e collo di un'olla. L'orlo ingrossato. Dm: $21 \mathrm{~cm}$. Argilla nero-brunastra, all'interno rossastra, micacea.

20. Orlo e collo di un'olla. L'orlo inclinato verso l'esterno è ingrossato, con estremità arrotondata e collo breve. ${ }^{290} \mathrm{Dm}: 14,3 \mathrm{~cm}$, la superficie è bruciata, con inclusioni nere (Fig. 19.4). (gruppo III)

21. Orlo perpendicolare, inclinato verso l'esterno. Dm: 10,8 cm. Argilla all'esterno marrone, leggermente micacea.

22. Fondo di un'olla. Argilla giallo-grigiastra, ben cotta, dura al tatto. (gruppo II)

\section{Dolium}

23. Fondo di un grande contenitore con corpo ovoidale e pseudo-piede ad anello. Dm (fondo): $14 \mathrm{~cm}$. Argilla ocra giallastra, ben cotta, dura al tatto (Fig. 20.1).

281 J. P. JONCHERAY: Etude d'épave Dramont “D” dite "des Pelvis" CahierArchSubaqu 3 (1974) pl.IV.

${ }^{282}$ Menchelli 2010, 245.

${ }^{283}$ L. QuILICI: Saggi di scavo attorno a Fidenae. NotScavi ser.8, 372, XXX. 1976, fig.43,507; Luni II. tav.270,3 Settefinestre tav.27,13; MENCHELLI 2003, fig.3,5. Abb. 17, 175 .

${ }^{284}$ Albintimilium 62, fig. 24,17 e 71; VEgas 1968,

${ }^{285}$ Menchelli 2010, Fig.5,13.
${ }^{286}$ Ostia, La taberna dell''Invidioso. Piazzale delle Corporazioni, portico ovest (saggi sotto i mosaici) a cura di M. Carta-I. Pohl-F. Zevi in: NotSc 1978 suppl al vol XXXII. 91, fig.96, n.96.

${ }^{287}$ Vegas 1968, Abb.13,136; Annese-De Felice-TurCHIANO 2000, fig. 11, 1 ? 2003, fig. 2,2

${ }^{288}$ VEGAS 1968, Abb.11,112; LUNI II. tav.271,7; MENCHELLI

${ }^{289}$ CARbonara-Messineo 1991, fig. 249,14.

${ }^{290}$ Vegas 1968, Abb.12,127. 


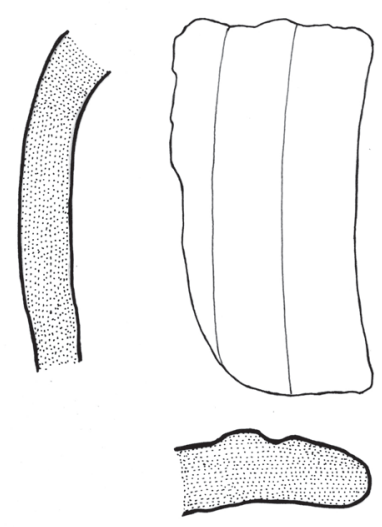

1
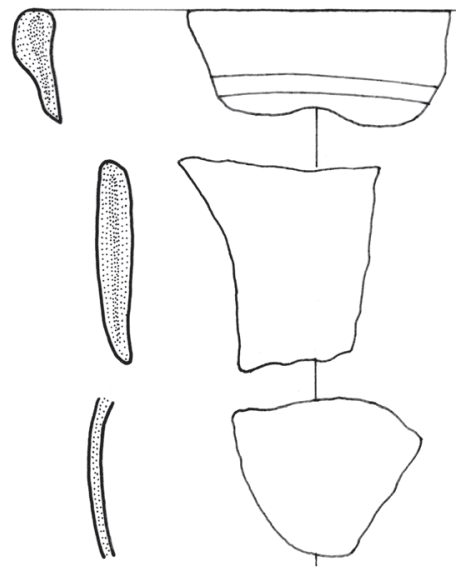

4
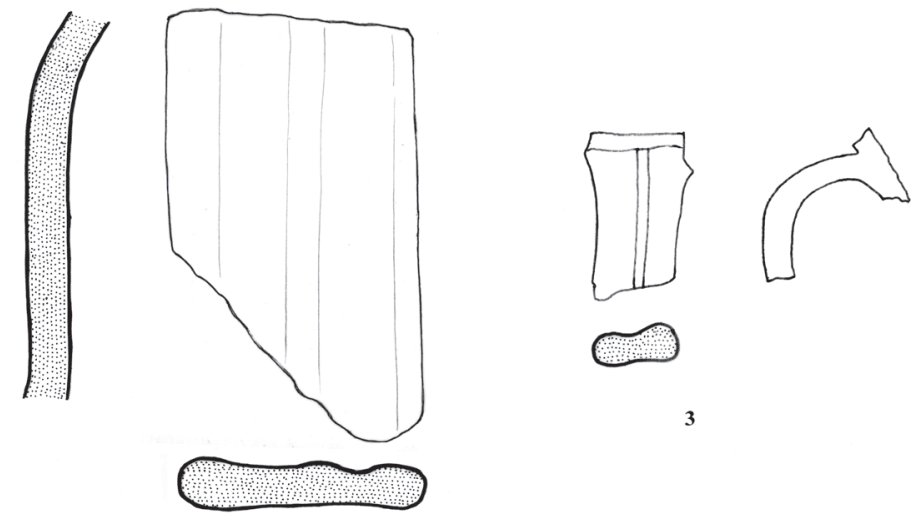

2
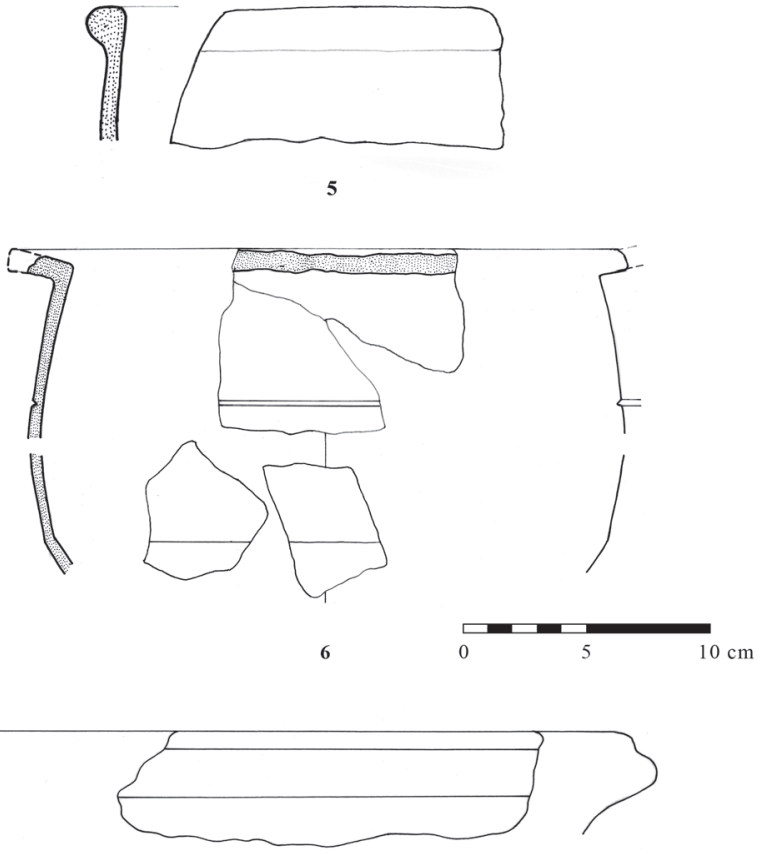

8
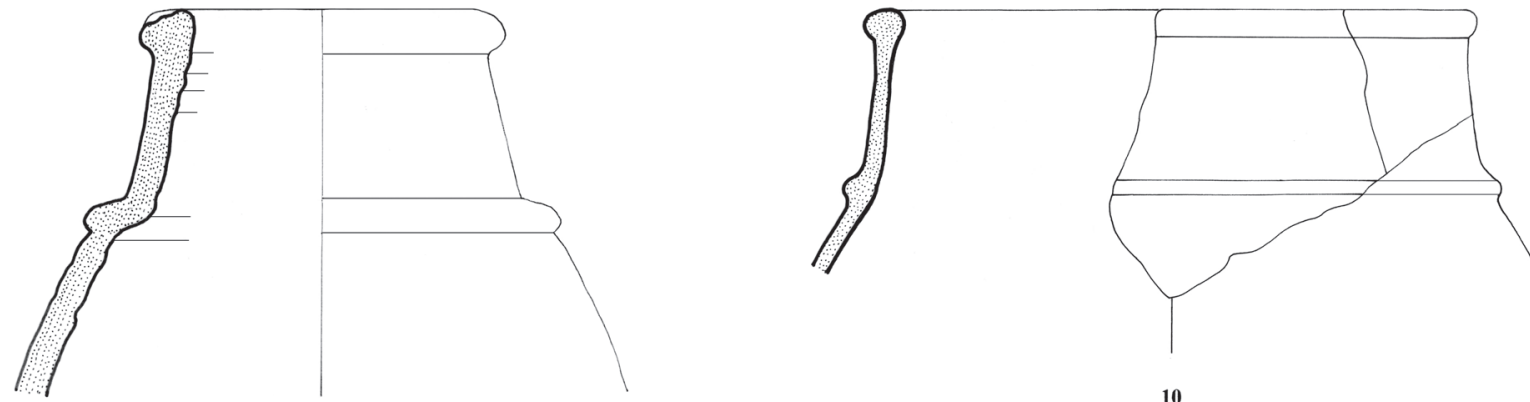

10

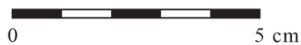

Fig. 18. Ceramica dallo strato d'incendio sito nella parte meridionale della villa più antica.

Acta Archaeologica Academiae Scientiarum Hungaricae 66, 2015 


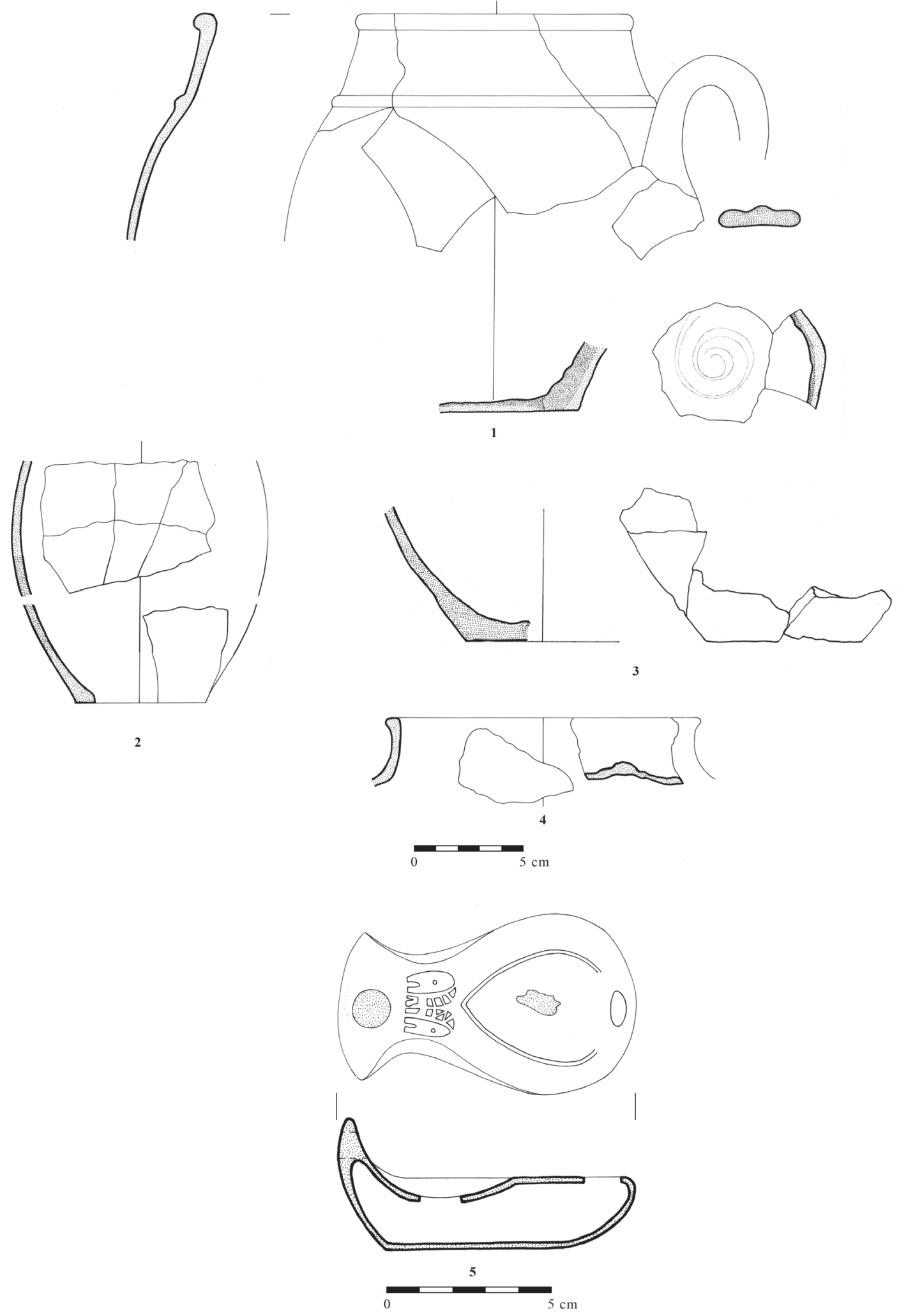

Fig. 19. Olle e lucerna dallo strato d'incendio sito nella parte meridionale delle villa più antica. 


\section{IV c Lucerne}

24. Lucerna integra di tipo Vogelkopflampe $=$ Dressel 22= Bailey $\mathrm{M}^{291}=$ Leibundgut XXVIII ${ }^{292}$.

In riferimento al tipo v. Deneavue tipo II293, De Caro fig.89,97294; K.Goethert-Polaschek: Katalog der römischen Lampen des Rheinischen Landesmuseums Trier. Bildlampen und Sonderformen. Trierer Grabungen und Forschungen 15. Mainz 1985, 13-15; A. Morillo-Garcia Marcos: Producciones cerámicas militares de época augusto-tiberiana en Hispania. Acta RCRF 37 (2001) 148-149

Disco liscio leggermente ribassato con infundibulum centrale (manca) con becco a ventaglio. Ansa triangolare, perforata, leggermente arrotondata. Base piatta. Tra il disco e il grande becco raffigurazione di due teste stilizzate di uccelli. Lungh. $9 \mathrm{~cm}$, largh: $5,6 \mathrm{~cm}$, alt. $4 \mathrm{~cm}$, base: $5,7 \mathrm{~cm}$. Argilla bianco-giallastra, presso il becco una macchia di combustione (Fig. 19.5, Fig. 26.2).

25. Fr. di disco e becco di una lucerna a testa d'uccello. Publ: D.Gabler-F.Redő: Nuove indagini nella villa romana di San Potito (Ovindoli, AQ). Quaderni di Archeologia d'Abruzzo 1 (2009) 111

Generalmente le lucerne di forma Dressel 22 sono trattate distintamente da un gruppo di Vogelkopflampe ${ }^{295}$ derivanti da un'epoca anteriore all'età repubblicana, pur essendo individuabili come la tipologia antecedente dei tipi d'età imperiale ${ }^{296}$. Quest'ultimo tipo, infatti, è più raffinato, di dimensioni minori e la decorazione stilizzata pure lo distingue dalle varianti precedenti della Vogelkopflampe ${ }^{297}$. Essendo questo tipo a Ostia presente in ogni strato di scavo attesta che fu prodotto assiduamente e per un lungo periodo. Forse i reperti rinvenuti negli strati più tardi possono essere ritenuti i residui più antichi, ma nel caso degli strati IV A e IV B questa ipotesi non pare verosimile giacché la proporzione degli stessi è grande ${ }^{298}$. Pur avendo in passato D. Bailey datato la durata della produzione di questa tipologia fino al I sec., tuttavia in base alle ricerche eseguite a Ostia si può ipotizzare che le produzione di queste tipologie abbia durato dall'età flavia fino alla metà del II sec. o fino all'età antonina nelle officine nell'Italia Centrale e Meridionale ${ }^{299}$.

Dal materiale rinvenuto a San Potito abbiamo pubblicato una simile lucerna venuta alla luce dalla colmatura sottostante al mosaico raffigurante una scena di caccia nel locale IX $^{300}$.

\section{IV $d$ Anfore}

Nello strato d'incendio abbiamo rinvenuto frammenti che si poté definire come pezzi di due anfore.

26. Base di un'anfora. La base compatta, che va allargandosi verso l'alto può essere probabilmente identificata come appartenente ad una forma Dressel 2-4. Dm: 3,3 cm, nel punto dove si allarga: 5,3 cm. Argilla giallastra, molto porosa, omogenea con granuli di quarzo (Fig. 20.,2).

Questa forma è nata su ispirazione delle anfore tardo-ellenistiche di Cos, alla fine del I sec. a. C. e poi vennero fabbricate anche durante i I e II sec d.C. sia in Italia, sia nelle provincie. ${ }^{301}$ Entro l'ambito italico fu prodotta

${ }^{291}$ D.M. BAILEY: Catalogue of the lamps of the British Museum II. Roman lamps made in Italy. London 1980, tipo M.

292 A. LeIBUNDGUT: Die römischen Lampen in der Schweiz. Bern 1977.

293 J. Denteavue: Lamps de Carthage. Paris 1969.

${ }^{294}$ S. DE CARO: Le lucerne dell'officina LVC. RendAccad Napoli 41 (1975) fig.89,97.

${ }^{295}$ M. RiCCI: Per una cronologia delle lucerne tardo-reppubblicane. Rivista di Studi Liguri 38 (1974) 205.

${ }^{296}$ C. PAVolini: Una produzione italica di lucerne: le Vogelkopflampen ad ansa traversate. BullComunale 85 (1976-77) 59-60.
${ }^{297}$ H. MENZEL: Antike Lampem im Römisch-Germanischen Zentralmuseum zu Mainz. Katalog des Römisch-Germanischen Zentralmuseums 15. Mainz 1954, 24.

${ }^{298}$ C. SAlONE in Ostia III. 396.

${ }^{299}$ R. SANTANGELI VALENZANi: Suppellettili di illuminazione. In: Settefinestre vol. 3, 1985, 212-213.

300 Zs. PONGRÁCZ: Lucerne in: Gabler-Redö 1994, 192, fig. 54

${ }^{301}$ B.BRUNO: Le anfore di trasporto in: D. GANDOLFI: La ceramica e i materiali di età romana. Classi, produzioni, commerci e consumi. Bordighera 2005, 375, 368-369. 
in Campania, Etruria, nell'area padana - cispadana e nei dintorni di Brindisi. ${ }^{302}$ Nel caso dei fr. la definizione è particolarmente difficile, ultimamente viene inquadrata a questo gruppo anche la forma Dressel 5, di cui si conoscono numerose varianti. Nel caso dei centri tirrenici sostituisce la forma Dressel 1, probabilmente per ragioni di praticità (capacità maggiore, comodità ed è anche più leggera). ${ }^{303}$ Si può qualificare come anfora vinaria in questo senso quasi una quarta parte dei nostri reperti di anfore rinvenuti frequentemente a San Potito. ${ }^{304}$ Pezzi di questo tipo sono stati trovati anche nel materiale del pozzo g-4. ${ }^{305}$ In base ai connotati qualitativi anche a San Potito se ne possono distinguere ben sette varianti, ma su questa base non sempre è possibile definirne la provenienza. Oltre alla provenienza italica si può eventualmente tener conto anche di esemplari derivanti dalla zona iberica.

27. Ansa di un'anfora Dressel 7-11 ? Ansa a nastro; la sezione per essendo ellittica è irregolare. Larghezza dell'ansa: 4,6 cm. Argilla color mattone, granuli di quarzo (Fig. 20.3).

\section{CERAMICA FUORI CONTESTO}

Nei seguenti si desidera presentare alcuni caratteristici tipi di ceramica, in casi in cui pur essendo sconosciute o incerte le relazioni stratigrafiche, tuttavia gli oggetti stessi rivestono una certa importanza dal punto di vista tipologico.

\section{V a Ceramica da mensa}

\section{Olpai}

1. Olpe con orlo a fascia distinto ricomposta da 9 frammenti tipo 6 secondo Olcese. Labbro estroflesso, sopra l'ansa un listello. L'orlo è concavo all'interno, collo troncoconico, ansa a nastro scanalata, impostata subito sotto l'orlo. Corpo ovoidale, piede ad anello. Alt. : $20 \mathrm{~cm}$, Dm dell'orlo: 4,6 cm, Dm del fondo: 6,2 cm. Argilla rossastra con sfumature più scure, ruvida, porosa non omogenea (Fig. 21.1, Fig. 26.4).

I confronti di questo tipo sono prima di tutto stati ritrovati a Ostia ${ }^{306}$. Nel caso del nostro esemplare la formazione dell'orlo è particolare, differente da quelli rinvenuti a Ostia, quindi non si può stabilire con sicurezza che si tratti di un prodotto di un'officina della città di Roma o ostiense. Gli esemplari rinvenuti ad Ostia possono essere datati al periodo tra l'epoca a proto imperiale fino al II sec., principalmente sulla base dei dati provenienti dalle Terme del Nuotatore ${ }^{307}$.Uno di questi venne alla luce da un edificio di epoca adrianea, ma fuori contesto. ${ }^{308}$ Nelle Terme del Nuotatore è ritrovabile nel I strato come residuo in un contesto databile alla metà del III sec. o anche a più tardi. L'esemplare di San Potito è stato rinvenuto il 10/10/1997, nell'ultimo vano verso $\mathrm{N}$ avente pavimento di coccio pesto della serie di ambienti occidentali del cortile centrale. Giacché sono venuti alla luce numerosi frammenti della brocca non è escluso che poté essere ancora in uso nel tardo periodo della villa, nel III sec.

\section{Brocca}

2. Brocca con orlo estroflesso, collo concavo, corpo ovoidale, ansa a bastoncello impostata sull'orlo e saldata sul ventre e sul fondo piano. Tipo 2 secondo Olcese. ${ }^{309}$ Alt.:17,1 cm, dm dell'orlo: 7,9 cm, dm del fondo: $5,4 \mathrm{~cm}$. Argilla marrone rossastra, poco porosa (Fig. 21.2, Fig. 26.5).

${ }^{302}$ Relativamente alla produzione italica v. C. PANELLA: Le anfore italiche del II secolo d.C. In: Amphores romaines et histoire èconomique, dix ans de recherche. Coll.École Française, Rome 114, 1989, 141-156; S. MARTIN-KILCHER: Die römischen Amphoren in Augst und Kaiseraugst. Ein Beitrag zur römischen Handels- und Kulturgeschichte 2. Die Amphoren für Wein, Fischsauce, Südfrüchte (Gruppe 2-24) und Gesamtauswertung. Forschungen in Augst 7/2. Augst 1994. 338-339.

${ }^{303}$ BRUNo 2005, 368-369.
${ }^{304}$ HÁRSHEGYI 2008, 136-137.

${ }^{305}$ HÁrshegYi 2008, 137.

${ }^{306}$ OlCESE 2003, tav.XXXI.2; Luni II. tav. 268,1; Settefinestre fig. 15,3

307 OLCESE 2003, 98.

${ }^{308}$ PAVOLINI 2000,19.

${ }^{309}$ Olcese 2003, tav.XXV.1; Vegas 1968, Abb.11,114; LuNI II, tav.267,11; Settefinestre fig.15,4. 


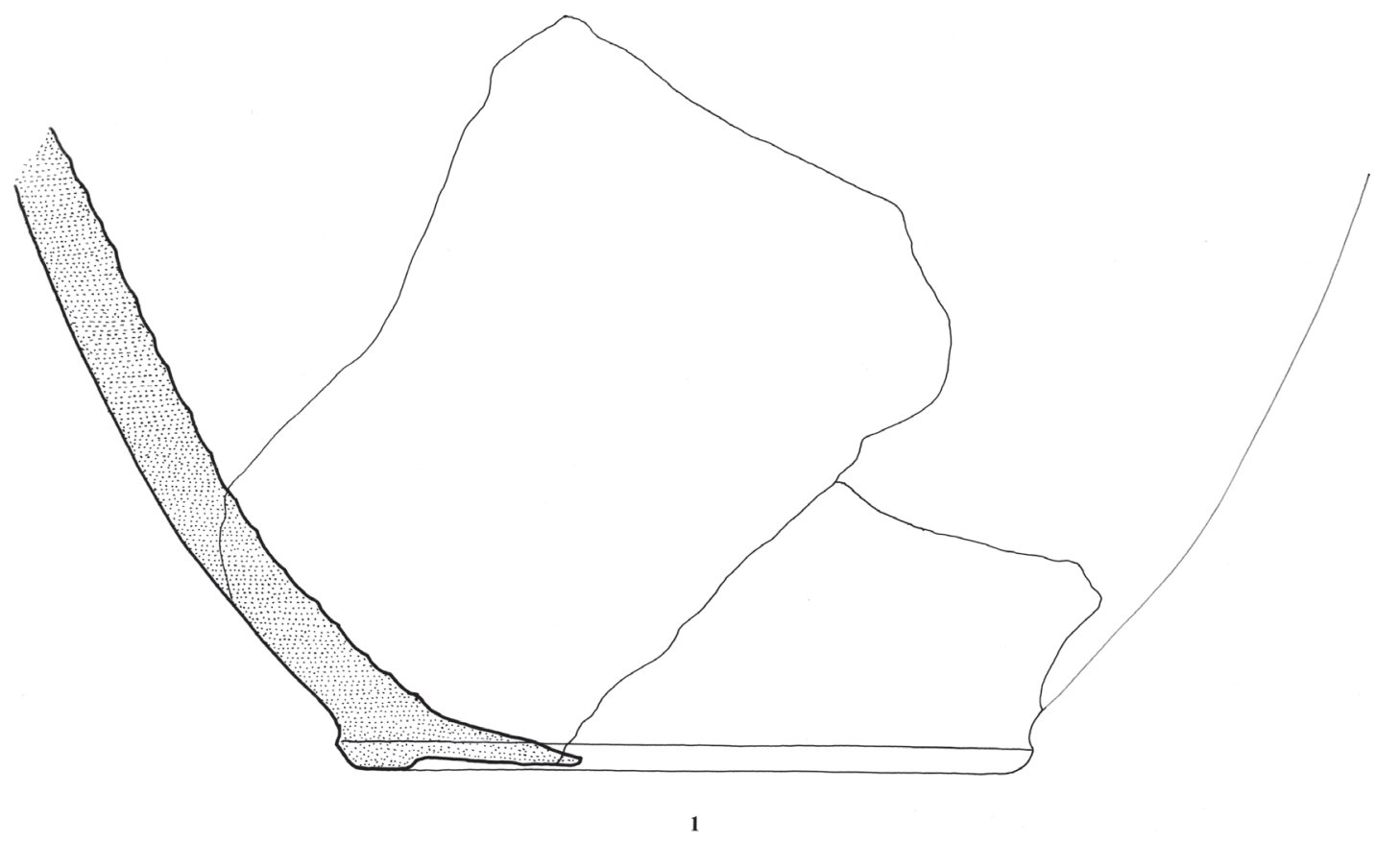

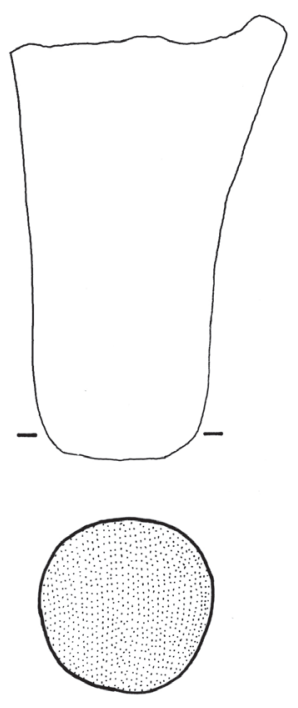

2

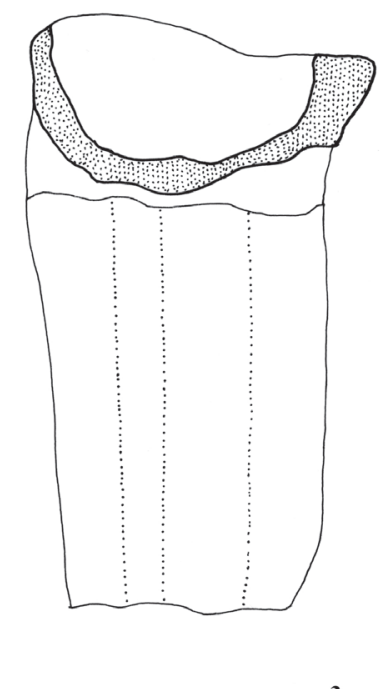

3
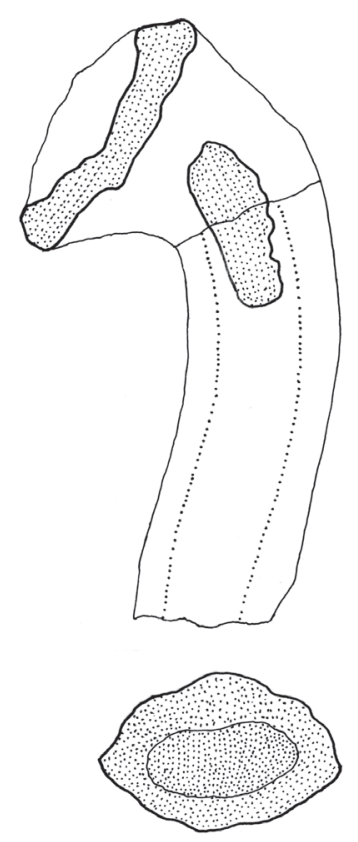

Fig. 20. Dolium e anfore dallo strato d'incendio sito nella parte meridionale della villa più antica.

Acta Archaeologica Academiae Scientiarum Hungaricae 66, 2015 


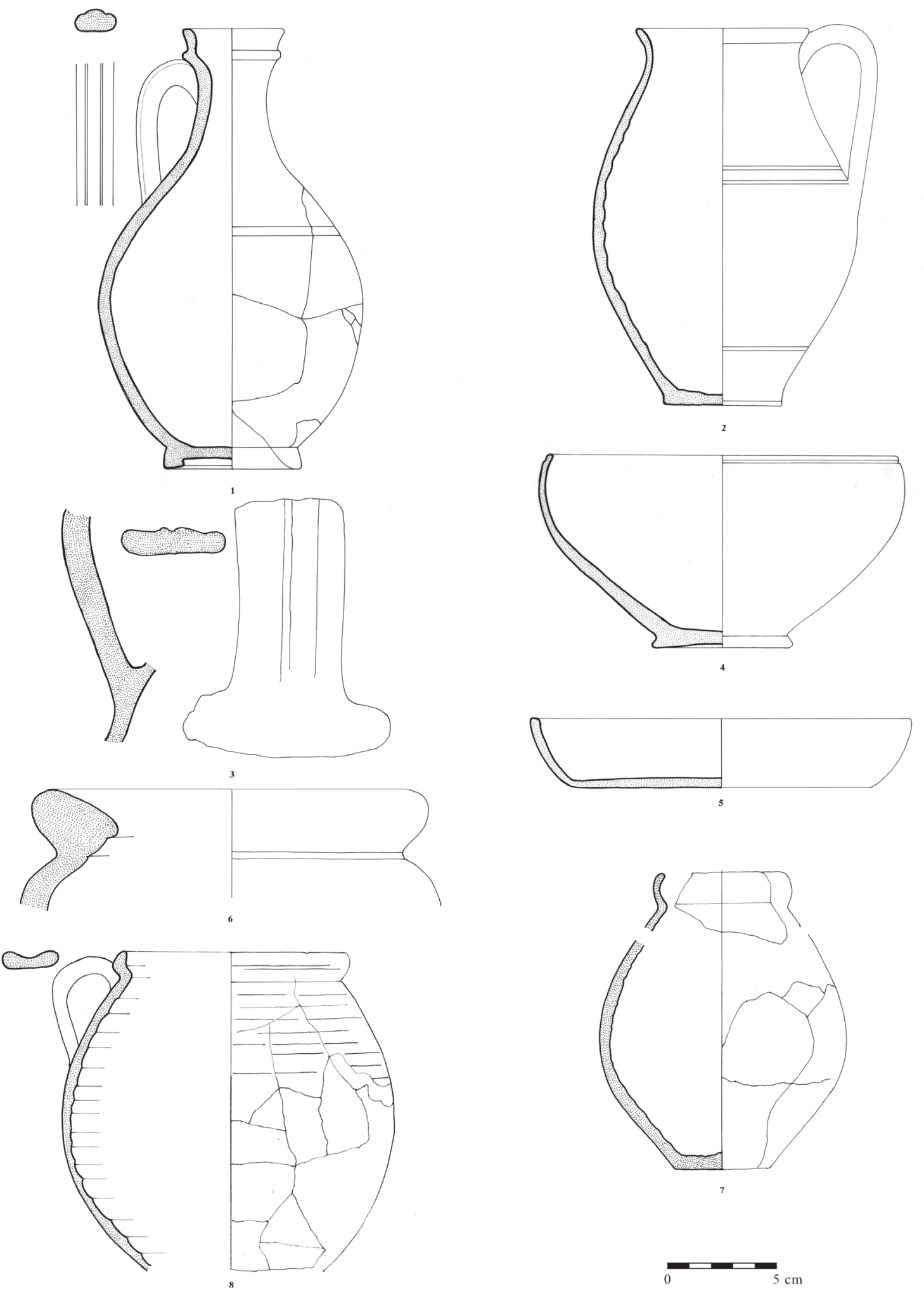

Fig. 21. Ceramica fuori contesto: olpai, brocca, coppa, tegame ed olle. 
Il tipo è ritrovabile ugualmente a Luni, Settefinestre, Gabii, Sutri e anche a Roma (La Celsa). Ė stato prodotto come lo attestano gli scarti rinvenuti nella fornace sita sul Gianicolo, a Roma e quelli di Sutri. La forma deriva dal I e dal II sec. ${ }^{310}$

Sito di rinvenimento: l'ultimo vano verso N, avente pavimento di coccio pesto, della serie occidentale di ambienti del cortile centrale (10/10/1997).

Si suppone che a San Potito poté essere in uso anche più tardi, essendo anche questo esemplare rinvenuto nello stesso sito dove l'olpe precedente, quindi non è escluso che fosse ancora in uso nel tardo periodo della villa, nel III sec.

3. Ansa di una brocca. L'ansa a nastro con tre solcature è saldata al ventre del vaso, la forma è Sutri $30 .{ }^{311}$ Argilla rosso-giallastra, porosa (Fig. 21.3)

Sito di rinvenimento: taglio u-5, fuori i muri, crollo di pietre, accanto al muro N-S dell'ambiente a pavimento coccio pesto $(19 / 09 / 1990)$

\section{Coppe con rivestimento}

4. Frammenti di una coppa emisferica; con incisione orizzontale, sul labbro ${ }^{312}$. Dm: $15,8 \mathrm{~cm}$. Argilla ocra giallastra, porosa, in fondo alla parte esterna rivestimento marrone scuro (Fig. 21.4).

A+11 taglio,fuori il muro SO della villa, riempimento del canale (5/10/1995)

5. Fr. di orlo e fondo simili al precedente Dm: 13,4 cm. Argilla ocra giallastra, porosa, all'esterno rivestimento marrone, all'interno si sono conservate solo macchie sparse dello stesso rivestimento.

B+11 taglio, fuori del muro di chiusura occidentale della villa nel riempimento del canale (5/10/1995).

\section{Tegame con rivestimento rosso}

6. Tegame ricomposto da 7 frammenti, manca la metà dell'orlo e della parete. L'orlo è introflesso all'estremità leggermente inclinato verso l'interno. Vasca con pareti bombate, a profilo arrotondato, fondo quasi piano. Tipo 4 secondo Olcese. ${ }^{313} \mathrm{Alt} .: 3,4 \mathrm{~cm}$, dm del orlo: $17,3 \mathrm{~cm}$, dm del fondo: $13,5 \mathrm{~cm}$. Argilla ocra scura, fondo annerito, rivestimento rosso interno, con macchie color marrone (Fig. 21.5).

La tipologia si è diffusa dall'età claudia e non solo in Italia, ma anche nelle province. Presente dal I sec. fino alla fine del II sec. La fine della produzione di questo tegame va posta alla fine del II e gli inizi del III sec. ${ }^{314}$

Questo esemplare è stato pure trovato il 10/10/1997, nell'ultimo vano verso N, avente pavimento di coccio pesto, della serie occidentale di ambienti dell' ala centrale della villa. Probabilmente si conservò quasi intatto rimanendo in uso anche nell'ultimo periodo della villa.

\section{$V$ b Ceramica da fuoco}

\section{Olle}

7. Olla a orlo estroflesso, arrotondato, con corpo ovoidale. La sezione molto grossa dell'orlo è triangolare ${ }^{315}$. Tipo 14 secondo O1cese. ${ }^{316} \mathrm{Dm}$ : 17,6 cm. Argilla ocra scura, leggermente porosa (Fig. 21.,6). Sito di rinvenimento: c+1, fuori i muri, accanto al canale, crollo di pietre $(30 / 09 / 1997)$

${ }^{310}$ OLCESE 2003, 93-94.

${ }^{311}$ DunCAN 1964, fig. 13, 118.

312 Menchelli 2012,34; Sutri forma 6, Duncan 1964,
${ }^{313}$ Olcese 2003, tav.XV.3; Vegas 1968, Abb.16,158; LUNI II, tav.263,1; Dyson 1976, LS 38; Settefinestre tav.31,1; ANNESE-DE FELICE-TurChiano 2000, tav. V, tipo 2.

${ }^{314}$ Settefinestre tav. 107

315 Settefinestre tav. 54,5.

${ }^{316}$ OLCESE 2003, tav. XIII.4. 
8. Olletta a corpo ovoidale ricomposta da 9 frammenti. Orlo estroflesso a profilo arrotondato; l'estremità inclinata verso l'interno (orlo a mandorla incavato internamente). Fondo piano. Tipo $3 \mathrm{~b}$ secondo Olcese. ${ }^{317}$ Tracce di tornio. Alt.: circa 11,9 cm, Dm (fondo) $4 \mathrm{~cm}$. Argilla rossa, ruvida con inclusi micacei, parete interna annerita, orlo annerito (Fig. 21.7, Fig. 27.2).

La tipologia è databile al periodo dall'epoca tardo-repubblicana fino al III sec.

Sito di rinvenimento: porticus fenestrata del cortile centrale, ambiente a $\mathrm{O}$ dall'ingresso della villa, accanto al canale (5/10/1998).

9. Olla monoansata con orlo estroflesso, l'estremità inclinata verso l'interno. Corpo ovoidale, la ansa a nastro impostata a collo. Tipo 6/7 secondo Olcese ${ }^{318} \mathrm{Dm}$ : 10,4 cm, alt: 14,6 cm + Argilla rosso-giallastra, porosa, bruciata, vicino all'annerita e dalla parte opposta all'ansa abbrunita dall'incendio (Fig. 21.8).

Questo tipo è ritrovabile in diversi siti, per es. a Sutri ${ }^{319}$, Gabii, Vasanello, Populonia, a Roma nella Villa di Livia, ne' La Celsa e a Ostia, dove è venuto alla luce in contesti datati al periodo tra gli anni 120-140, ma praticamente fu prodotto durante tutto il II sec. A San Potito l'abbiamo rinvenuto nello strato di detriti della villa, quindi è ipotizzabile che il tipo fosse in uso anche nel III sec.

Sito di rinvenimento: verso Sud dall'ambiente XV, corridoio di forma L, strato d'incendio soprastante il pavimento a mosaico.

10. Fr. di olla a pareti inarcate con orlo sporgente a forma di goccia, forma Sutri $27^{320}$. Argilla giallo-rossastra, porosa, micacea (Fig. 22.1). Sito di rinvenimento: taglio 9 a, crollo di mattoni, $70-75 \mathrm{~cm}$

\section{Pentole}

11. Fr. dell'orlo di una pentola? a pareti spesse. All'interno l'orlo sporgente e verticale è concavo. Forma Sutri 51. ${ }^{321}$ Argilla color giallo ocra, porosa. Sito di rinvenimento: taglio 16, a E dal muro, sotto il pavimento del praefurnium, 75-80 cm (6/10/ 1984) (Fig. 22.2)

12. Fr. di una pentola con orlo ingrossato a tutto tondo. Argilla ocra brunastra, porosa. Sito di rinvenimento: taglio 17 a, humus con crollo, 40-45 cm (13/10/1985) (Fig. 22.3)

13. Fr. di una pentola con orlo a tesa, forma Sutri $44 .{ }^{322}$ Argilla ocra giallastra, ben cotta, dura al tatto. Sito di rinvenimento: taglio 16, a E dal muro, sotto il pavimento del praefurnium, 75-80 cm (6/10/1984) (Fig. 22.4)

14. Pentola con orlo a tesa di forma Sutri $24 .{ }^{323} \mathrm{Dm}$ : $18 \mathrm{~cm}$ Argilla rosso-giallastra, porosa. Sito di rinvenimento: taglio 9 a, strato di crollo di mattoni, 70- $75 \mathrm{~cm}$ (Fig. 22.7).

15. Pentola con orlo a tesa, forma Sutri 20. Argilla giallo-ocra, porosa. Sito di rinvenimento: taglio $17 \mathrm{a}$, humus con crollo, $40-45 \mathrm{~cm}$ (13/10/1998). (Fig. 22.6)

\section{Tegami}

16. Fr. dell'orlo di un tegame a pareti spesse. La sezione dell'orlo sporgente è pentagonale. Forma Sutri $17 .{ }^{324}$ Argilla gialla tendente al rosso, porosa. Sito di rinvenimento: taglio 17 a, humus con crollo, 40-45 cm (13/10/1985). (Fig. 22.5)

17. Tegame con pareti grosse e orlo a goccia. ${ }^{325}$ Argilla rosso-giallastra, porosa, all'interno grigia. Sito di rinvenimento: taglio 17 a, humus con crollo, 40-45 cm (13/10/1998).

18. Tegame ricomposto da 4 frammenti con parete inclinata verso l'esterno; l'estremità inclinata verso l'interno. Fondo piano, tipo 4 secondo Olcese. ${ }^{326}$ Alt. : 4 cm, fondo: $14,5 \mathrm{~cm}$. Argilla all'interno rossa, micacea, all'esterno marrone (Fig. 22.8, Fig. 27.1)

Questa tipologia è ritrovabile a Ostia, Sutri, Viterbo, deriva dai sec. I e II. Sito di rinvenimento: ambiente XVII, SE angolo, strato di crollo (1995).

${ }^{317}$ Olcese 2003, tav.VIII.7; Vegas 1968, Abb.14,138; BonNet 2001, fig.3,6; ANNESE 2000, 10.5.

${ }^{318}$ OlCESE 2003, tav.X.5, 186; Vegas 1968, Abb.13,136; LuNI I. tav.61, 26 M, 1230/9; DYsON 1976, fig.49,88.

${ }^{319}$ DunCAN 1964, fig. 7,3.

${ }^{320}$ DunCAN 1964, fig. 12,102; Dyson 1976, fig.44, 17.
${ }^{321}$ DunCAN 1964, fig.16,187?; Dyson 1976, LS 11.

${ }^{322}$ DunCAN 1964, fig. 15, 170.

${ }^{323}$ DunCAN 1964, fig. 10,82; CAStel GiubiLeo fig.43,493.

${ }^{324}$ DunCAN 1964, fig.9,62; Dyson 1976, fig.44, 17.

${ }^{325}$ DYSON 1976, fig.46,38.

${ }^{326}$ OLCESE 2003, tav.XV.3. 

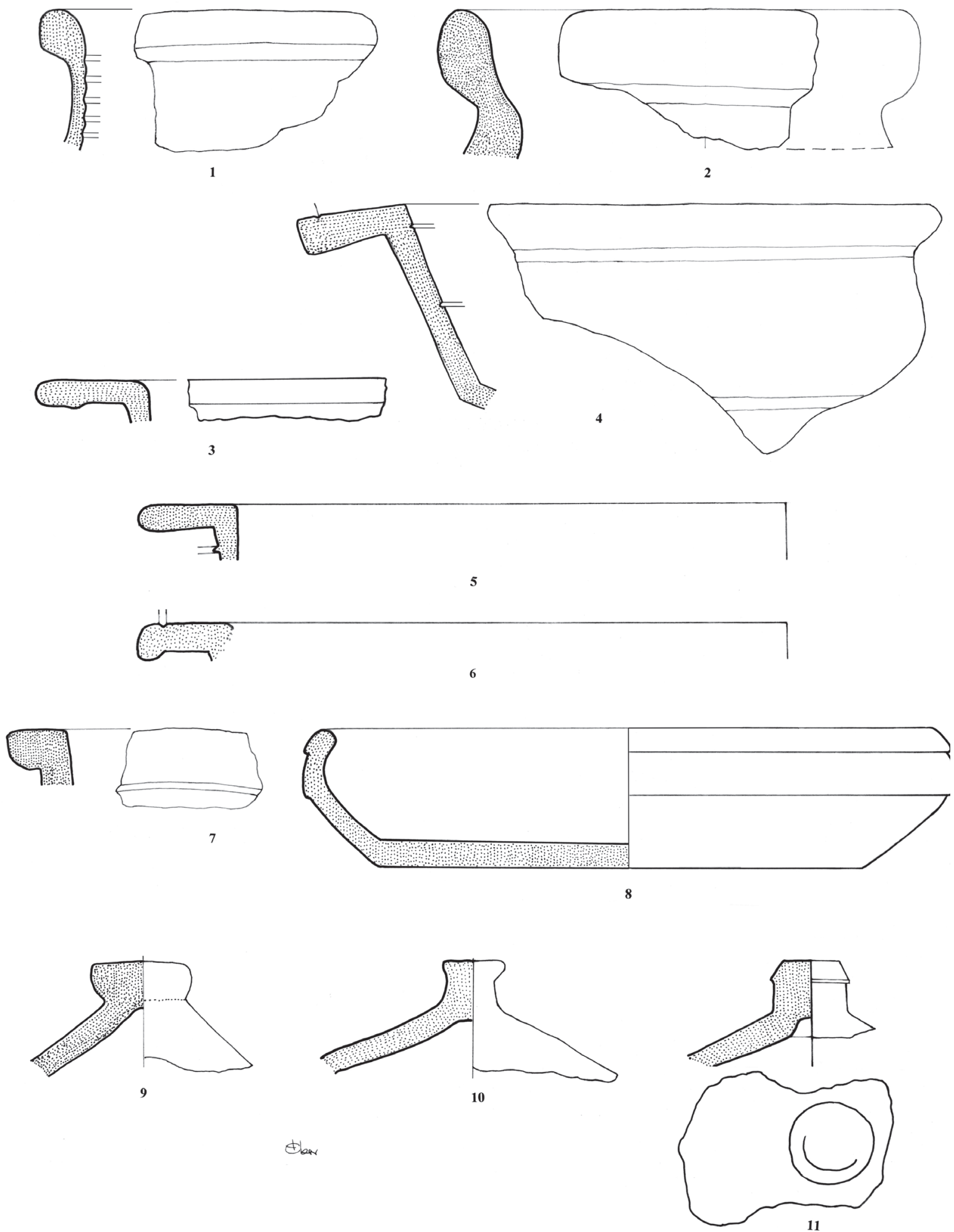

Elear

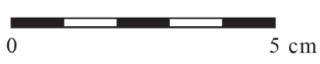

Fig. 22. Ceramica fuori contesto: pentole, tegami e coperchi. 


\section{Coperchi}

19. Fr. di coperchio troncoconico con manico rotondo, sporgente, forma Sutri $48 .{ }^{327}$ Argilla marrone giallastra, leggermente porosa. Sito di rinvenimento: taglio 17 a, humus con crollo, 40-45 cm (15/10/1985). (Fig. 22.9, Fig. 27.5)

20. Fr. di coperchio troncoconico con manico cilindrico che va allargandosi in alto forma Sutri $48^{328}$. Argilla giallo-rossastra, porosa. Sito di rinvenimento: taglio u-5, fuori i muri, crollo di pietre, accanto al muro N-S dell'ambiente a pavimento coccio pesto (19/09/1990).(Fig. 22.10)

21. Fr. di coperchio troncoconico con manico in alto pure troncoconico forma Sutri $48 .{ }^{329}$ Argilla giallo-rossastra, porosa. Sito di rinvenimento: cortile centrale, angolo SO, lungo il muro EO, verso Est dal canale, strato di crollo (19/10/ 1998) (Fig. 22.11).

22. Fr. di coperchio piatto con orlo rientrante, v. Dyson 1976, LS 55. Argilla giallo-rossastra, porosa. Sito di rinvenimento: taglio $\mathrm{n}+3$, fuori i muri della scalinata, crollo di pietre (7/10/1988).

\section{$V$ c Ceramica per la preparazione}

\section{Bacini}

23. Fr. di un bacino con orlo estroflesso. Sull'orlo una solcatura Collo troncoconico; tra il collo e la vasca un spigolo ${ }^{330}$. Tipo 2 secondo Fabbri. Dm: $36 \mathrm{~cm}$. Argilla giallo-rossastra, porosa. Sito di rinvenimento: Parte SO del cortile centrale, lungo il muro E-O, verso l'Est dal canale, strato di crollo (19/10/1998). (Fig. 23.1)

Abbiamo già presentato questo tipo nella pubblicazione riguardante i reperti venuti alla luce dal riempimento del canale che porta alla cisterna situato al centro del cortile settentrionale. Databile probabilmente al I sec.

24. Fr. d'orlo di un bacino simile al precedente, con listello sottile sotto l'orlo. Dm: 32,4 cm. Sito di rinvenimento taglio 17 a nella parte orientale della villa, humus mescolato con crollo, $40-50 \mathrm{~cm} .($ Fig. 23.2)

\section{Mortaria}

25. Mortarium a vasca arrotondata, orlo a profilo triangolare arrotondato, due prese impostate sull'orlo, colatoio e granuli interni, fondo piano. Sull'orlo un bollo illeggibile. ${ }^{331} \mathrm{Dm}$ : circa. $29 \mathrm{~cm}$, alt.: $8,7 \mathrm{~cm}$. Argilla ocra giallastra, porosa. Sito di rinvenimento: c+1, nel cortile centrale, accanto al canale, crollo (1/10/1997). (Fig. 23.3;Fig. 27.4)

\section{Incensieri}

26. Orlo estroflesso con decorazione ondulata. Sulla vasca a cordonatura motivi a forma di spina. Tipo 2 secondo Olcese. ${ }^{332}$ Argilla rosso-giallastra, porosa. Sito di rinvenimento: taglio c+1, strato di crollo, accanto al canale, (30 sett.1997). (Fig. 23.,4)

27. Fr. di un incensiere. Sito di rinvenimento: porticus fenestrata, accanto al canale, strato di crollo (15/10/1998). (Fig. 23.5)

\section{Lucerne}

Nei seguenti si pubblicano tutte le lucerne e tutti i frammenti di lucerne rinvenuti a San Potito.

1) Fr. di una lucerna Vogelkopflampe .Manca gran parte del disco ribassato. Becco di forma a ventaglio. Base piana. Tipo Dressel 22= Leibundgut XXVIII= Bailey O-P= Pavolini III.1= De Caro fig.89,97. Alt.: 2,1 cm, dm (becco): 4,5 cm. Argilla bianco-giallastra. Sito di rinvenimento: taglio c+1, accanto al pilastro (29/09/ 1997) (Fig. 24.1)

2) Lucerna integra di tipo Loeschcke VIII.L.1= Dressel 20-21=Deneauve VII a= Cerulli Irelli gruppo I. ${ }^{333}$ Sul disco liscio concavo lacuna, manca l'infundibulum. Spalla larga delimitata da una modanatura. Grande becco corto, cuoriforme. Ansa forata, base piana. Dm: $7 \mathrm{~cm}$, lungh.: 10,2 cm, alt.: $2 \mathrm{~cm}$. Corpo ceramico di colore arancio tendente al bruno. Sito di rinvenimento: verso l'Ovest dall'ingresso principale (7/10/1998) Questo tipo fu prodotto dalla fine del I sec. fino allo scorcio tra il II e III

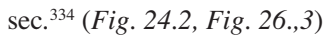

${ }^{327}$ DunCAN 1964, fig. 15,177; Dyson 1976, LS 92, fig.62.

${ }^{328}$ DunCAN 1964, fig. 15,177-178.

${ }^{329}$ DUNCAN 1964, fig. $15,178$.

${ }^{330}$ Dyson 1976, fig.49,78 22 II; FABBri 2008, fig.4,3.

${ }^{331}$ Sui bolli v. S. PALLECHi: I mortaria di produzione centro-italica: corpus dei bolli. Roma 2002.
332 OlCESE 2003, tav.XXI.6; DysOn 1976, 224, fig.50,104.

${ }^{333}$ G. Cerulli Irelli: Officine di lucerne fittili a Pompei. Quaderni di cultura meteriale 1. L'instrumentum domesticum di Ercolano e Pompei nella prima metà imperiale. Roma 1977.

${ }^{334}$ Ostia III. 399; LeIBUNDGUT 1977, 39-40. 

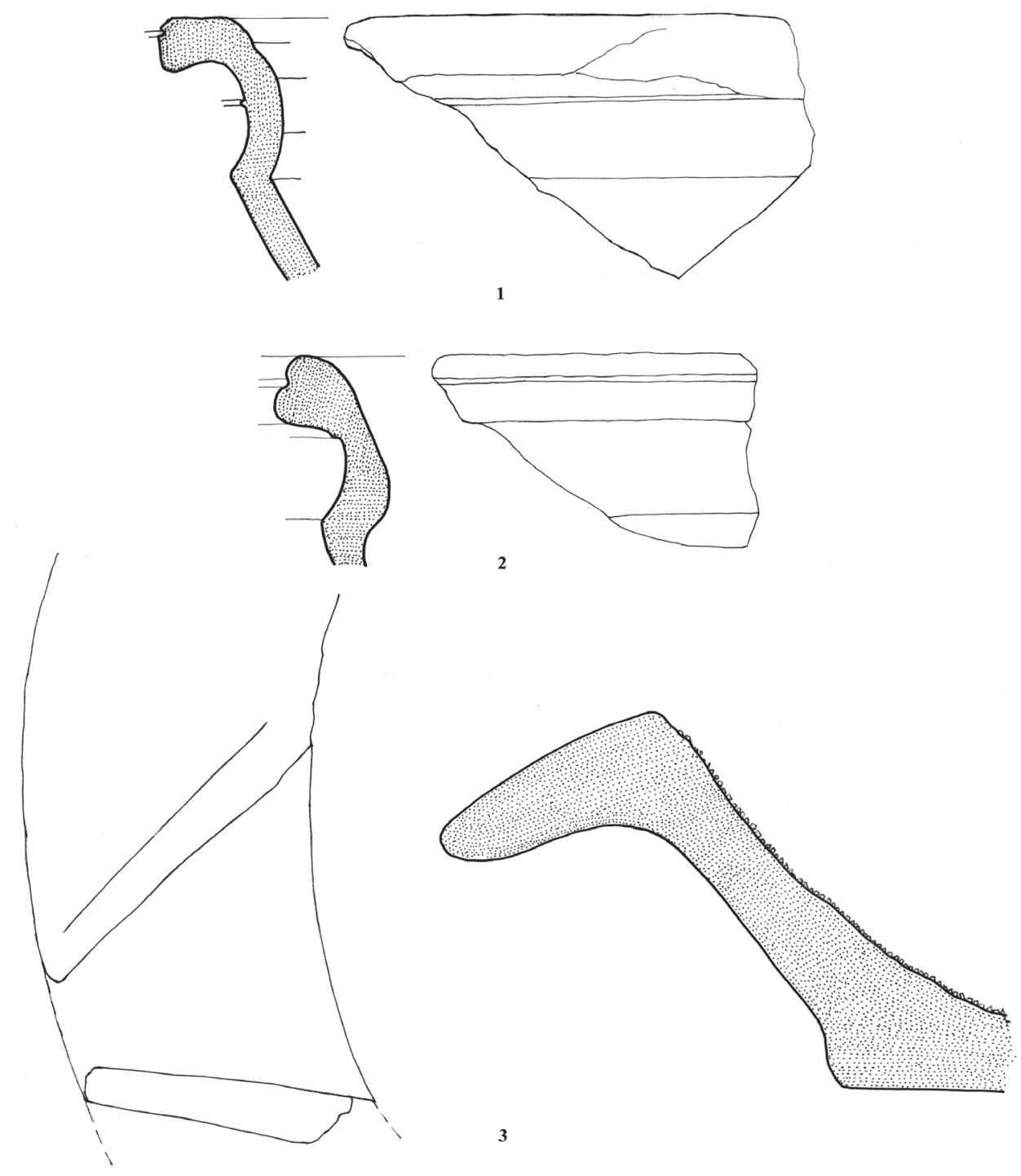

3
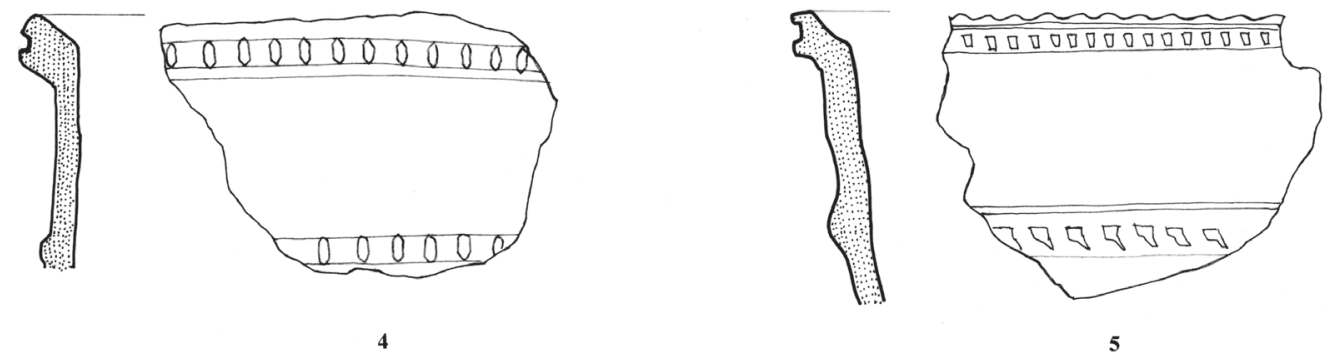

4

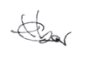

Fig. 23. Ceramica fuori contesto: bacini, mortarium ed incensieri.

Acta Archaeologica Academiae Scientiarum Hungaricae 66, 2015 

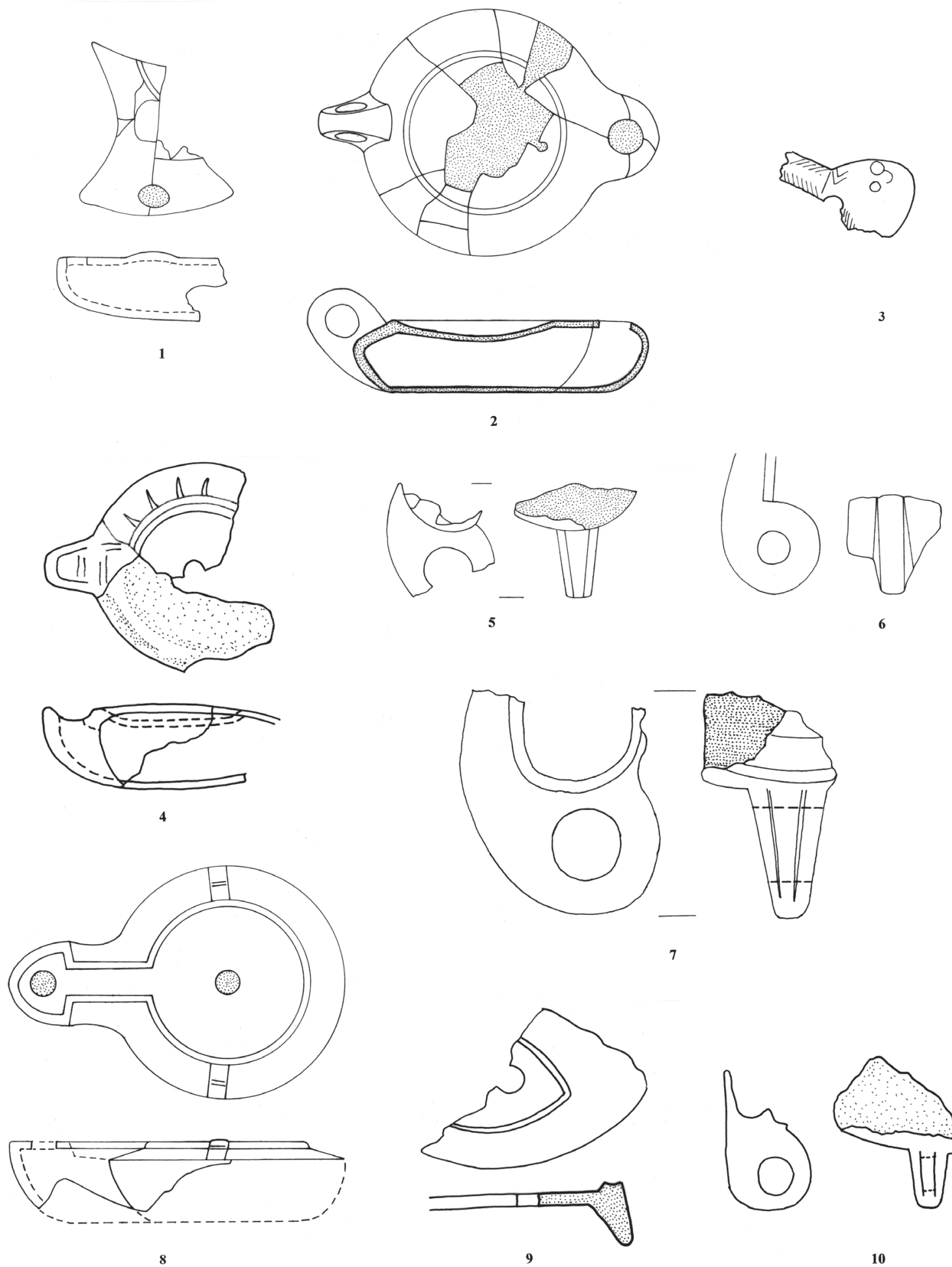

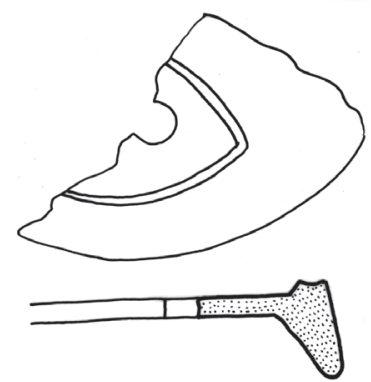

9
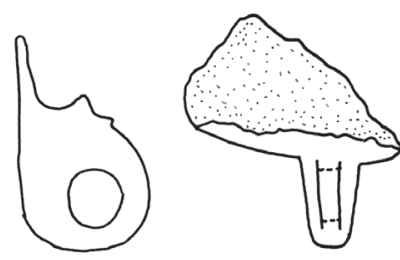

10

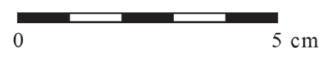

Fig. 24. Lucerne fuori contesto. 
3) Fr. di una lucerna di tipo Loeschcke VIII H=Dressel 20-21=Deneauve VIII A=Heeres Ee ${ }^{335}$ di cui manca parte dell'ansa e disco. Spalla larga estroflessa, delimitata da due solchi, becco corto cuoriforme. Base piana. Alt.:2,5 cm Dm: 8,4 cm. Corpo ceramico ocra, la vernice bruna abrasa. Sito di rinvenimento: taglio d+11, saggio fuori di muri, accanto all'apertura del canale, sotto il basamento, crollo (2/10/1995) (Fig. 24.,3)

4) Fr. del disco e fondo di una lucerna di tipo Loeschcke VIII L.1=Dressel 20-21=Deneauve VII A=Cerulli Irelli gruppo I. Posto su un disco liscio, concavo, limitato da due circonferenze incise. La spalla è decorata da linee radiali. Parete sottile. Becco limitato da una linea retta. Dm: 6,3 cm. Corpo ceramico ocra, vernice bruno-rossa abrasa. Sito di rinvenimento: Ambiente XXVII, lungo il muro settentrionale, a $55 \mathrm{~cm}$ dal vertice del muro, crollo di mattoni e pietre (Fig. 24.,4)

5) Fr. dell'ansa forata di una lucerna di tipo Dressel 20-21. Argilla arancio con resti di vernice rossa. Sito di rinvenimento: c+1, accanto al pilastro (29 sett.1997) (Fig. 24.5)

6) Fr. dell'ansa forata di una lucerna di tipo Dressel 20-21. Argilla ocra. Sito di rinvenimento: a+4-5-6, crollo (1/10/1997). (Fig. 24.,6)

7) Fr. dell'ansa forata di una lucerna di tipo Dressel 20-21. Sporadico (2/10/1997).

8) Ansa forata di una lucerna di tipo Dressel 20-21. Dm (dell'ansa): 6,4 cm. Argilla ocra, vernice marrone. Sito di rinvenimento: cortile centrale, sopra il canale, crollo (16/10/ 1998). (Fig. 24.7)

9) Parte superiore di una lucerna di tipo Buchi $\mathrm{X}$ a ${ }^{336}=$ Dressel 5=Deneauve IX A= Leibundgut XXX-XXXIV= Bailey 1980, N. Disco liscio con infundibulum centrale. Lievi scheggiature sull'anello di spalla. Sulla spalla due borchiette piramidali. Sul canale aperto foro di aerazione. Sul becco non ci sono tracce di combustione. ${ }^{337}$ Lungh. 9,8 cm, dm: 6,5 cm. Argilla ocra tendente all'arancio. Sito di rinvenimento: taglio c+1, fuori i muri, accanto al canale (29/09/ 1998). L'inizio della produzione di questo tipo è intorno al 100 d.C. La produzione continua nel II e III sec. d. C. ${ }^{338}$ (Fig. 24.8)

10) Fr. di disco e fondo di una lucerna a volute? Loeschcke tipo I=Iványi tipo I=Dressel-Lamboglia tipo 9=Deneauve tipo IV A=Leibundut tipi V-VIII. Corpo ceramico ocra, vernice rossa, abrasa. Sito di rinvenimento: Nymphaeum nel centro del cortile settentrionale, strato di crollo di pietre, $120-150 \mathrm{~cm}$ (8/10/1991) (Fig. 24.9.)

11) Fr, di fondo di una lucerna. Argilla giallo-ocra, farinosa. Sito di rinvenimento: A+10, verso Nord dal muro E-O, 180-200 cm, sotto alluvione (11.10.1995).

12) Becco ? di una lucerna. Argilla giallo-ocra. Sito di rinvenimento: saggio N-S dell'asse del cortile settentrionale, sporadico $(11 / 10 / 2000)$.

13) Fr. del disco di una lucerna. Argilla rosso-giallastra, vernice bruna abrasa. Sito di rinvenimento: lungo il muro perimetrale dell'abside, nella parte esterna, crollo.

14) Fr. di disco di una lucerna. Argilla rosso-giallastra. Sito di rinvenimento: $\mathrm{m}-2$, ambiente accanto alla scala, pavimento battuto, combustione $(2 / 10 / 2001)$.

15) Fr. di disco di una lucerna. Dm: $5,6 \mathrm{~cm}$. Argilla ocra giallastra, porosa. Sito di rinvenimento: buco della fognatura, ubicato fuori i muri perimetrali, humus antico con mattoni, sopra il basamento (29/09/1995).

16) Fr. di disco di una lucerna con infundibulum. Argilla ocra giallastra. Sito di rinvenimento: $B+2+3$, saggio verso 1'Ovest dall'ultimo pilastro (30/09/1997).

17) Fr. di ansa forata di una lucerna. Argilla: ocra (Fig. 24.10). Sito di ritrovamento c+11, d+11, verso sud dalla costruzione di mattoni (2.ott.1995).

Tra le lucerne troviamo relativamente molti tipi Dressel 20-21, le cosiddette Vogelkopflampe, mentre, contrariamente alle nostre aspettative determinate dalla loro situazione cronologica, mancano le lucerne con voluta. La quantità delle Firmalampen o lucerne a canale che rimasero in uso per tutto il II sec., è pure bassa.

${ }^{335}$ G. HEERES: Die römischen Bildlampen der Berliner Antiken-Sammlung. Berlin 1972.

${ }^{336}$ E. Buchi: Lucerne del Museo Nazionale di Aquileia I. Lucerne romane con marchio di fabbrica. Aquileia 1975.
${ }^{337}$ A. LARESE: Le lucerne fittili e bronzee del Museo Concordiese di Portogruaro. Roma 1983, no.89.

${ }^{338}$ LeIBUNDGUT 1977, 47. 

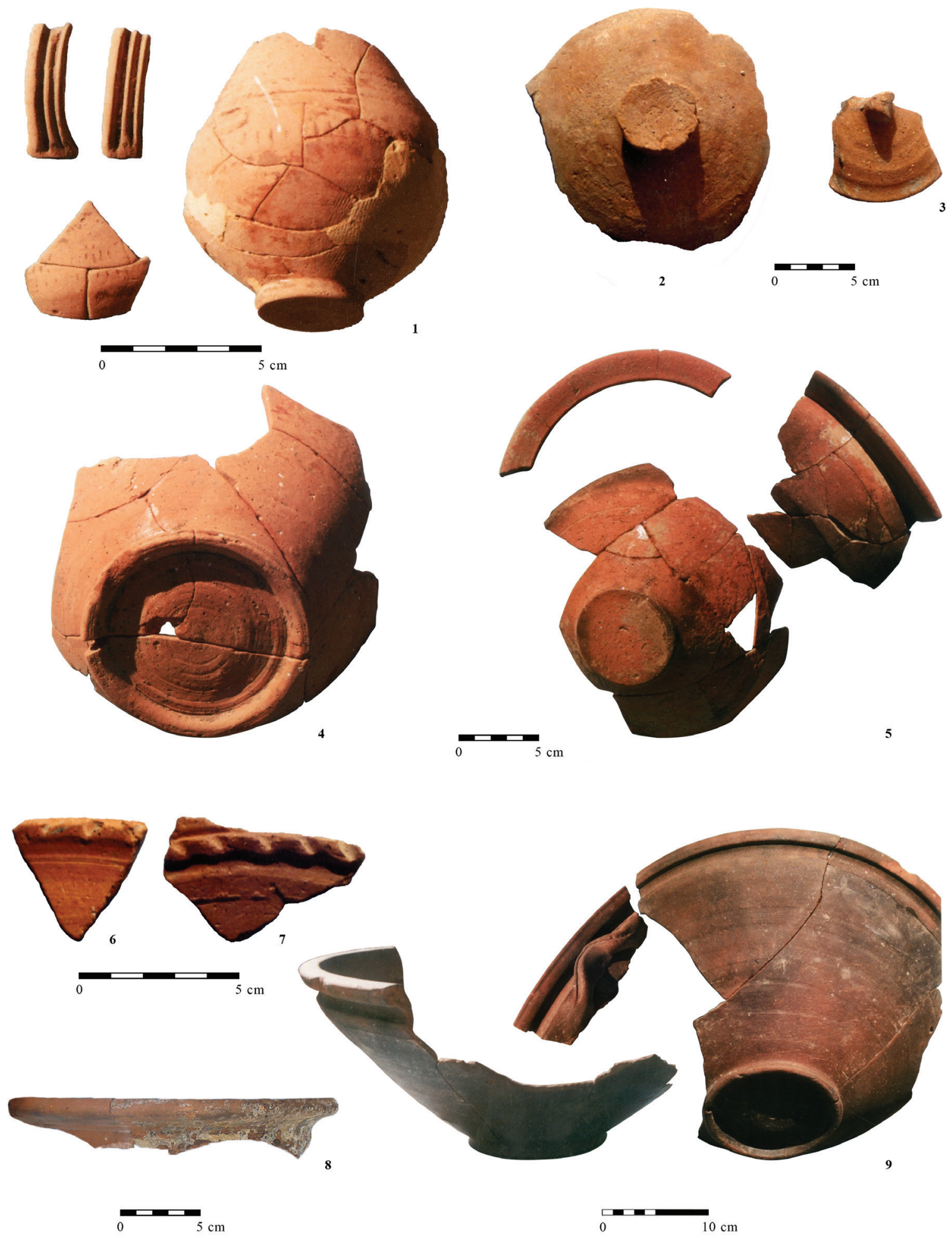

Fig. 25. Ceramica a pareti sottili (1) rinvenuta nell'ambiente ubicato ad Ovest dal ingresso principale della villa (secondo periodo) e ceramica comune rinvenuta nel pozzo nel taglio g-4 $(2-4,6-7)$ e nella cisterna al centro del cortile settentrionale $(5,8-9)$. 


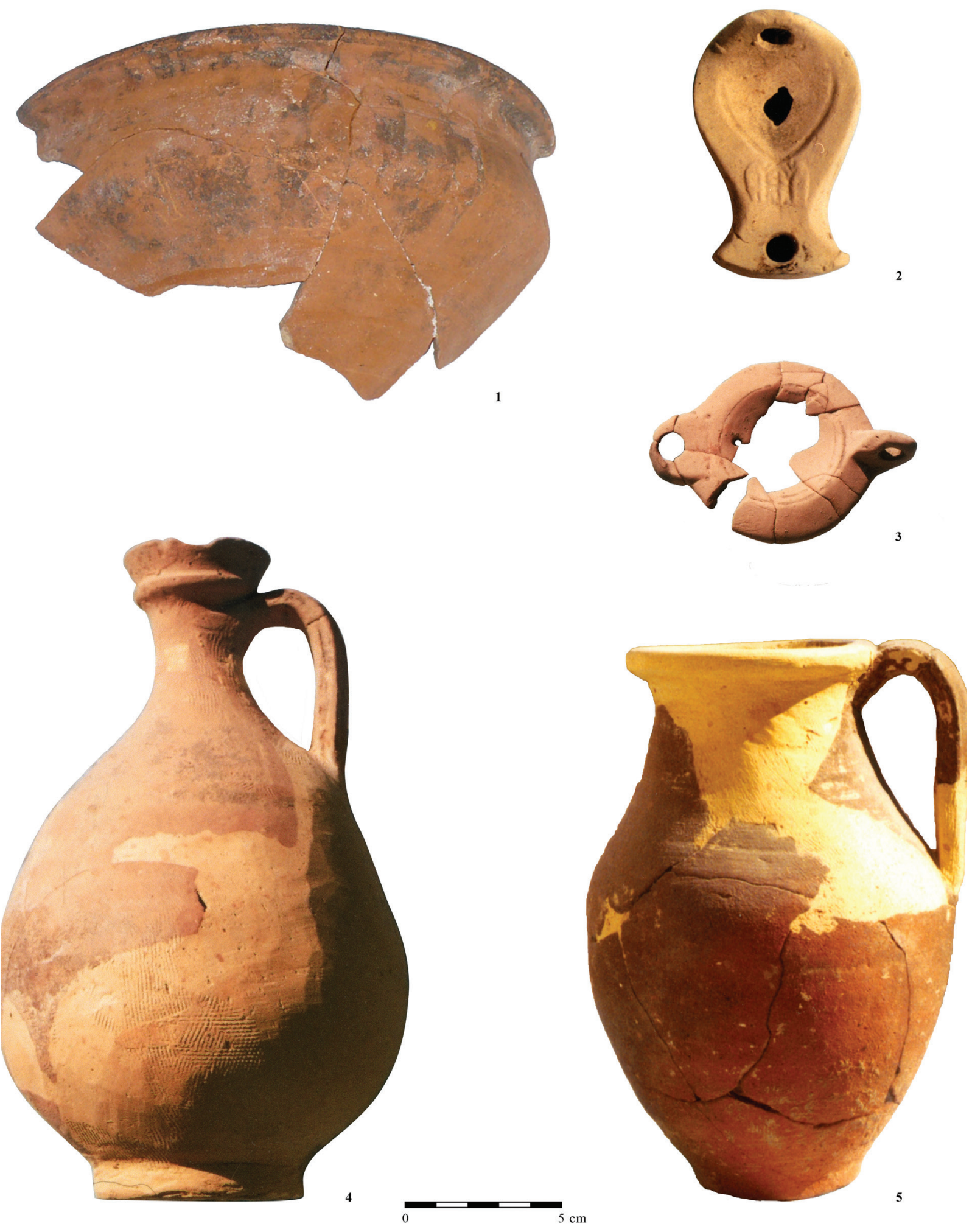

Fig. 26. Ceramica rivestita rinvenuta nello strato d'incendio (1),

lucerna rinvenuta nello strato stesso (2), lucerna (3) e ceramica comune rinvenuta fuori contesto (4-6). 

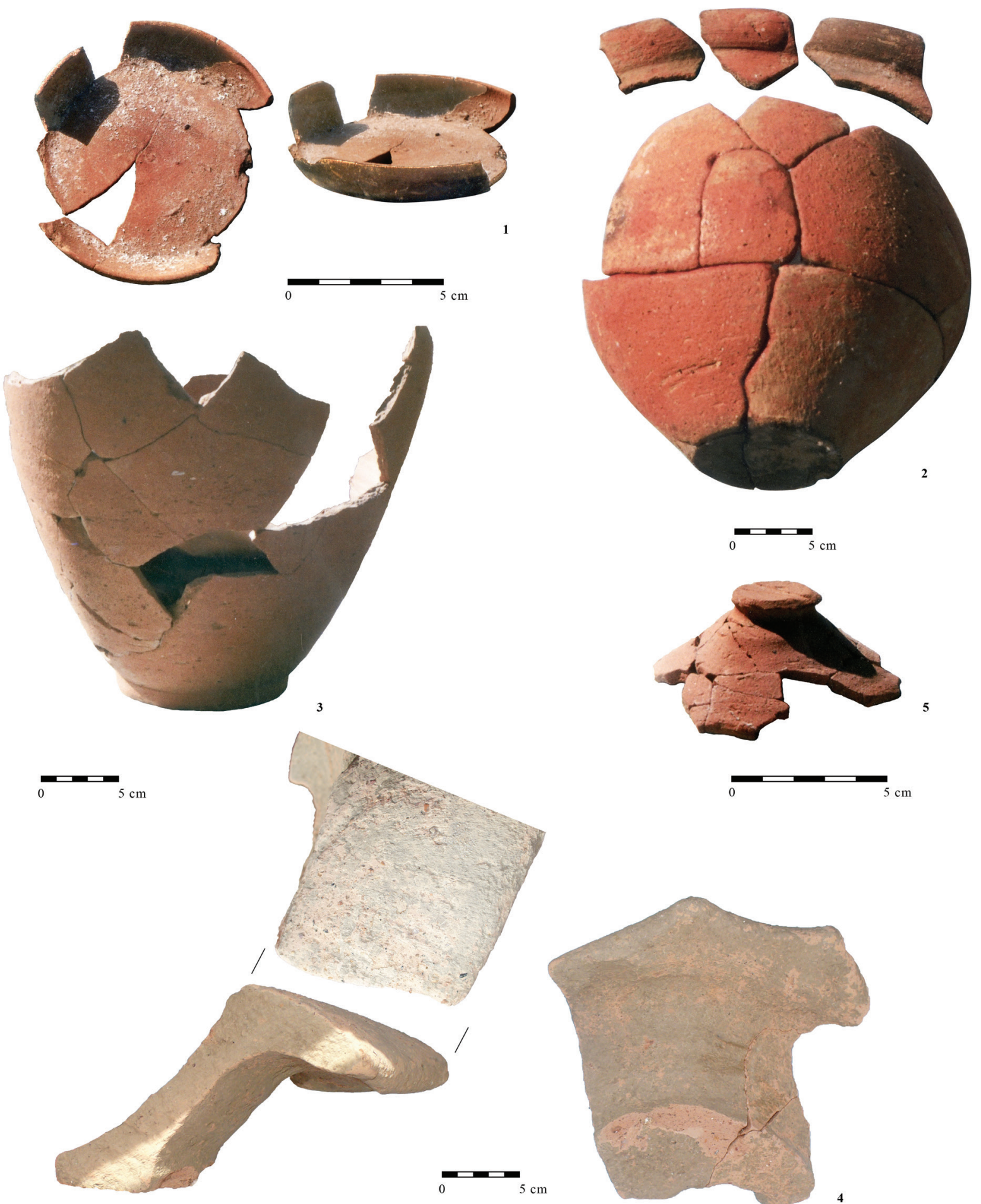

Fig. 27. Ceramica comune fuori contesto 
CONCLUSIONI

La quantità della ceramica a pareti fini e della ceramica importata, utilizzabile per la datazione è piuttosto limitata.

Dal pozzo scavato nel taglio g-4 abbiamo portato alla luce quattro sigillate che furono pubblicate già precedentemente. ${ }^{339}$ Ogni vaso è di produzione italiana, una coppa di forma Consp.29 e due Consp.34.1, nonché un piatto di forma Consp.20.4. Le due coppe di tipo Consp.34.1 vennero alla luce da profondità diverse, una era situata a $250 \mathrm{~cm}$ e l'altra a $440-480 \mathrm{~cm}$. Oltre alle sigillate si può ipotizzare che la coppa carenata di vetro di forma Isings $2^{340} \mathrm{e}$ i frammenti di una bottiglia siano prodotti di officine italiche.

Si poté definire precisamente tre delle cinque anfore; una di forma Dressel 2-4 serviva per il trasporto del vino e probabilmente fu prodotta in un'officina campana, ma non è escluso che fosse un contenitore di vino di Rodi o di Cnido ${ }^{341}$ nella seconda metà del I sec. d.C. o all'inizio del II. L'unico esemplare che può essere ritenuto indubbiamente ceramica importata è l'anfora di tipo Dressel 14=Lusitanian Garum I, fabbricata nelle officine della valle di Sado che probabilmente giunse alla villa contenendo per l'appunto salsa di pesce, cioè del garum. ${ }^{342}$ Similmente si può qualificare come anfora da garum un vaso di forma Beltrán II B che giunse a San Potito dalla Spagna.

Nel riempimento della cisterna e del relativo canale, scavati nel centro del cortile N, abbiamo rinvenuto solamente un frammento di vasellame importato, si tratta di una ceramica giunta dall'Africa Settentrionale di forma Hayes 8 che, in base ai connotati qualificativi, può essere attribuita al gruppo A $1 .{ }^{343}$ Probabilmente questo tipo fu prodotto alla fine del I sec. e nella prima metà del II sec. nella Tunisia del N., forse nelle vicinanze di Cartagine. Questo esemplare conferma la nostra ipotesi secondo la quale per colmare la cisterna e il canale che vi conduce che nella seconda metà del III sec. non erano più in funzione si dovette usare un deposito di scarti che conteneva frammenti di ceramica di un'epoca antecedente.

La ceramica databile, rinvenuta nella parte $\mathrm{S}$ della villa più antica, può essere collocata circa all'età flavia, eccetto il frammento dell'unica ceramica a vernice nera che, probabilmente deriva dal primo terzo del I sec. ${ }^{344}$ L'unica terra sigillata italica rinvenuta in questo luogo ha la forma di Consp.3.1. È databile in un arco di tempo ampio che va dalla metà del I sec. alla metà del II. ${ }^{345}$ L'unica sigillata che giunse nella villa dall'estero nell'età di Nerone e Vespasiano ${ }^{346}$ è una coppa di La Graufesenque, di forma Drag.29. Uno dei due frammenti di anfore rinvenuti nello strato d'incendio ha la forma di Dressel 2-4 e forse anche questo poté esser prodotto in un'officina campana, mentre l'altro ha forse la forma di Dressel 7-11. Se questa definizione è giusta, allora l'anfora probabilmente fu usata per trasportare garum proveniente dalla Spagna.

Il più grande contesto "chiuso" è stato rinvenuto nel riempimento del pozzo del taglio g-4, in cui abbiamo rinvenuto 390 frammenti di vasellame, ma la proporzione degli esemplari di ceramica importata (provenienti da territori extraitalici) era solo del mezzo percento. Oltre alle due anfore spagnole anche nel caso dell'anfora di forma Dressel 2-4 si può eventualmente azzardare la supposizione che si tratti dell'importazione di vini provenienti da Cnido o Rodi; comunque, nemmeno in tal modo la quantità non raggiunge in totale l'1 percento. La proporzione della ceramica fine rinvenuta nel pozzo è del $6,4 \%$; di cui la maggior parte è ceramica a pareti sottili mentre la quantità della ceramica rivestita, a cui si può inquadrare vasi di tipi differenti, è minore (Fig. 28). La tipologia di terre sigillate, tutte di provenienza indubbiamente italica, è presente nella minor quantità. Probabilmente sono state prodotte in officine centro italiche. Alcuni confronti compariscono a Roma; gli esemplari decorati a rilievo, invece, sono stati fabbricati probabilmente nell'Etruria Settentrionale, ${ }^{347}$ tuttavia si può tener conto anche di Corfinio, ${ }^{348}$ mentre pare poco probabile che il loro luogo di produzione fosse Scoppieto o Vasanello.

Il $91,4 \%$ dei nostri reperti appartiene al tipo della cosiddetta ceramica comune. Oltre a queste due categorie di grande rilievo, (ceramica fine (Fig. 29), ceramica comune) sono presenti tre altre categorie rappresentate però solo da alcuni esemplari: le anfore $(1,2 \%)$, le ampolle $(0,5 \%)$ che, servivano come contenitori di profumi, creme,

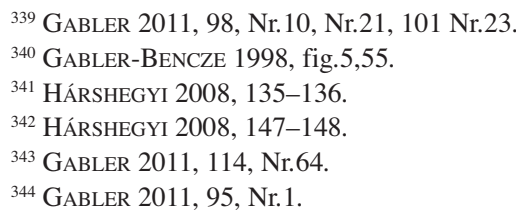

${ }^{339}$ Gabler 2011, 98, Nr.10, Nr.21, 101 Nr.23.

${ }^{344}$ GABLER 2011, 95, Nr.1.

${ }^{345}$ GABLER 2011, 103, Nr.34

${ }^{346}$ GABLER 2011, 112, Fig. 7 .

${ }^{347}$ GABLER 2011, 108

${ }^{348}$ G. Rizzo: Samia etiamnunc in esculendis laudantur (Pl.N.H. XXXV. 160-161: vasi “aretini” a Roma. MEFR 110 (1998) 799-848. 


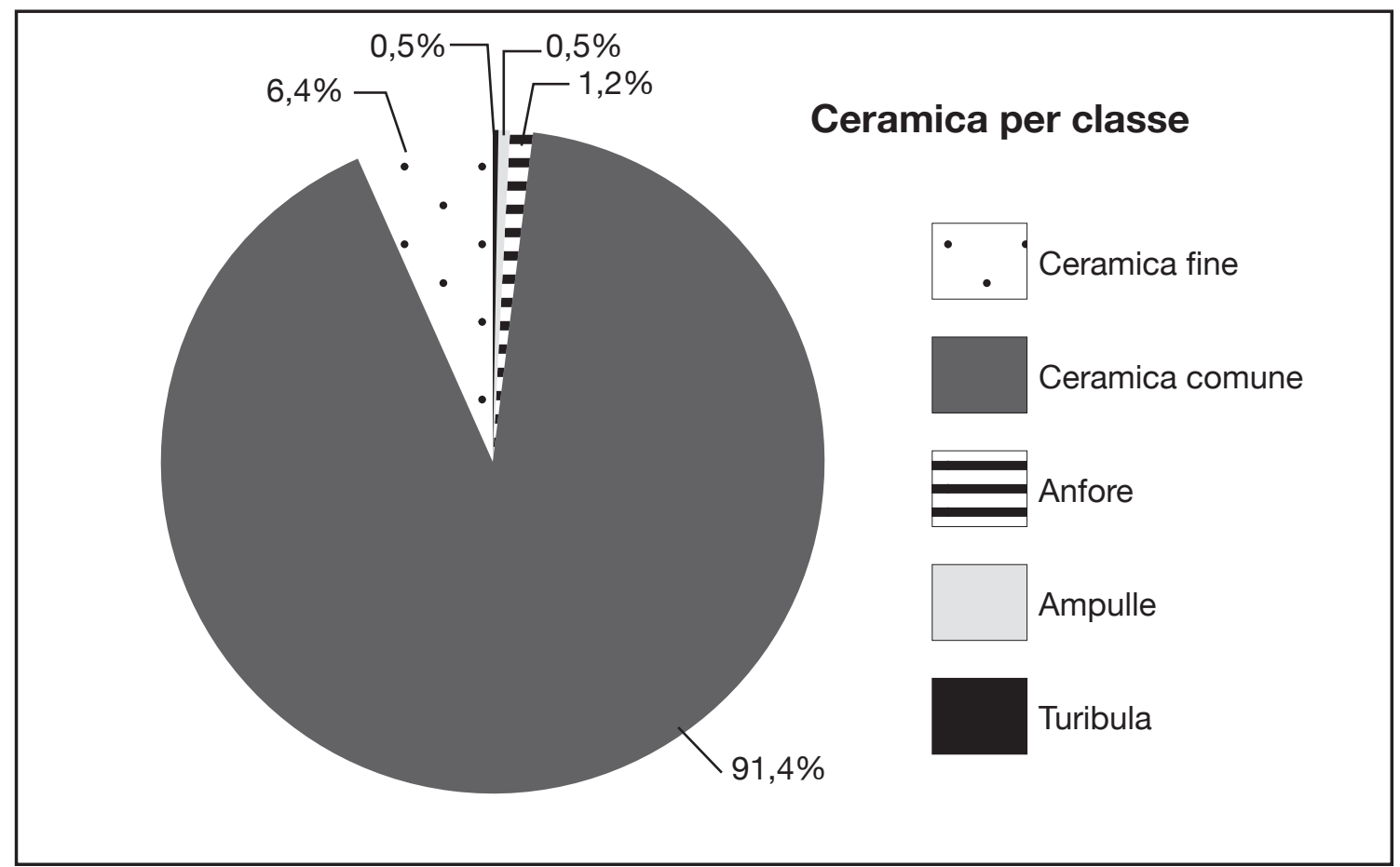

Fig. 28. Distribuzione percentuale della ceramica rinvenuta nel pozzo del taglio g-4.

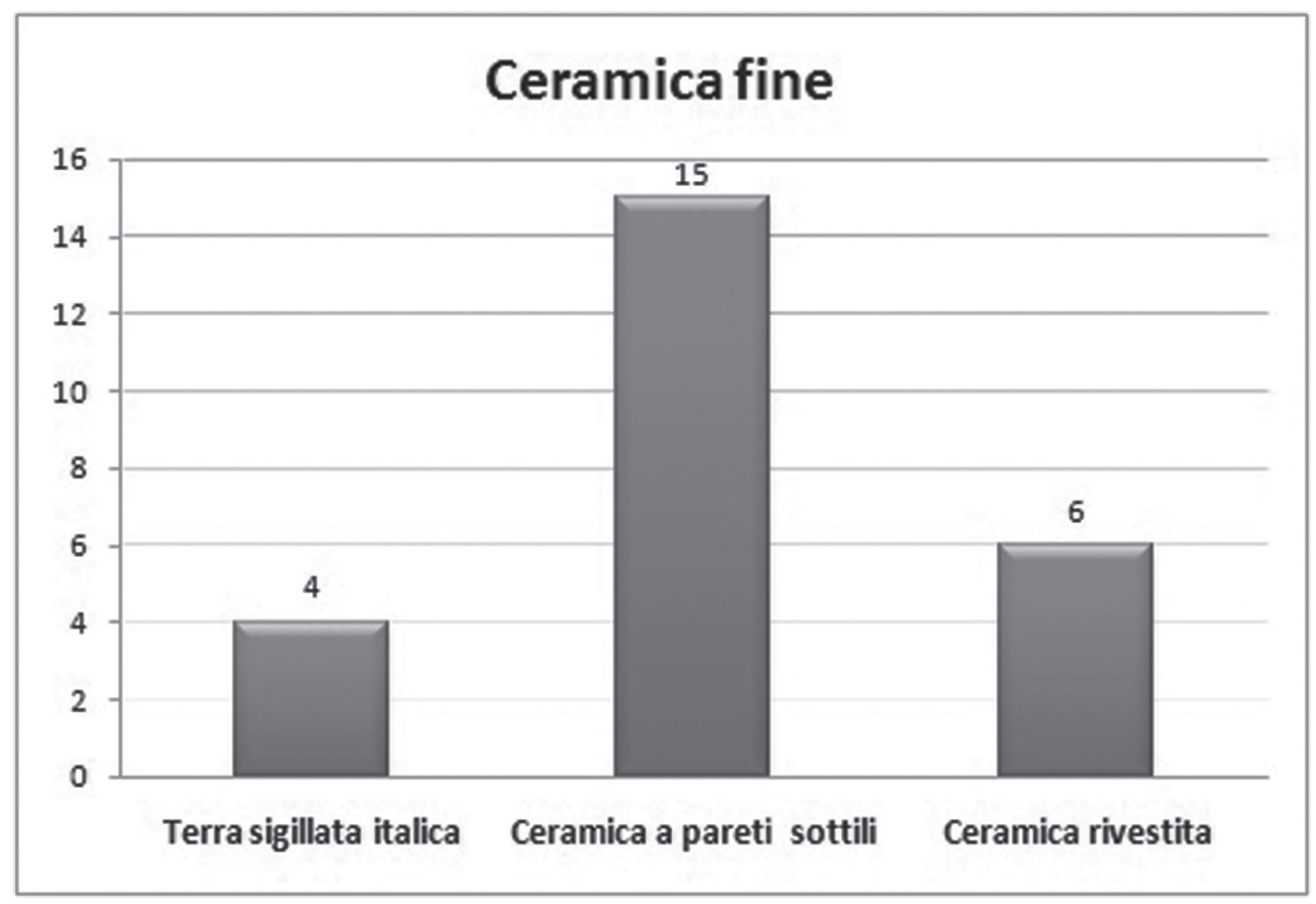

Fig. 29. Distribuzione della ceramica fine rinvenuta nel pozzo del taglio g-4. 


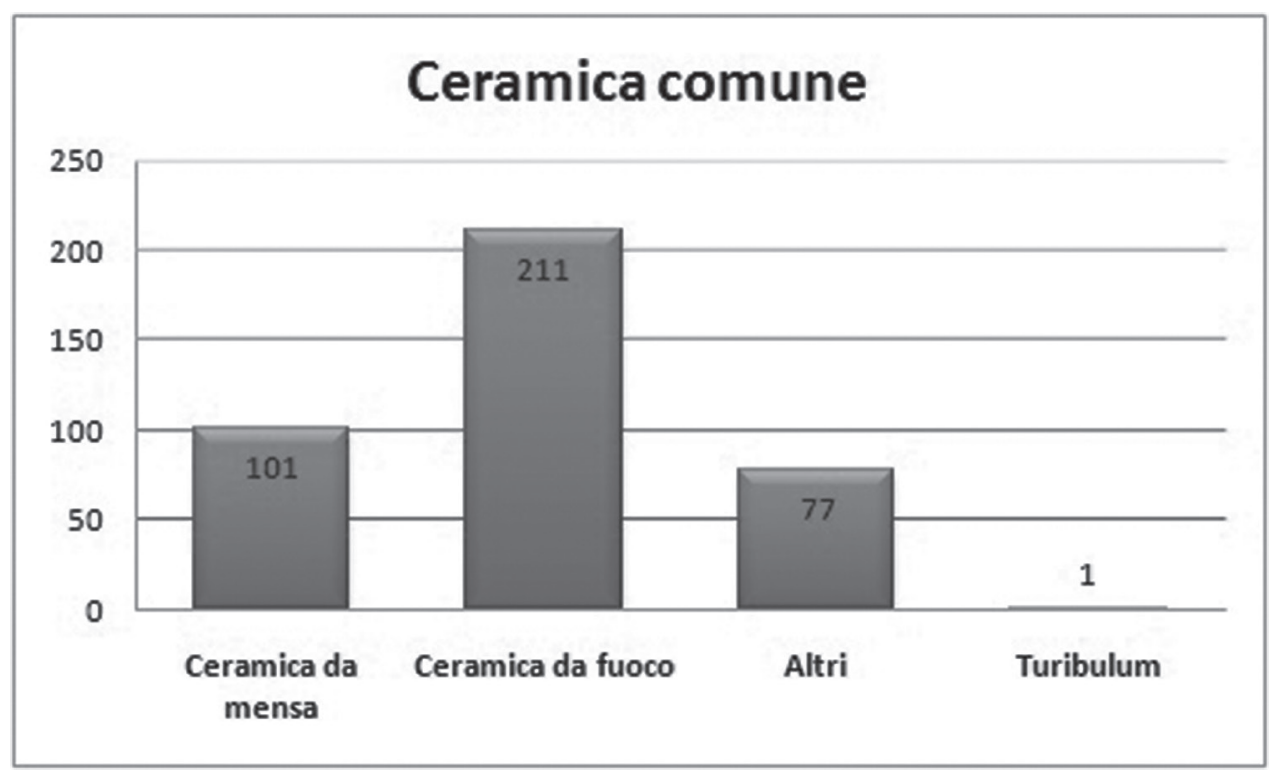

Fig. 30. Distribuzione della ceramica comune rinvenuta nel pozzo del taglio g-4.

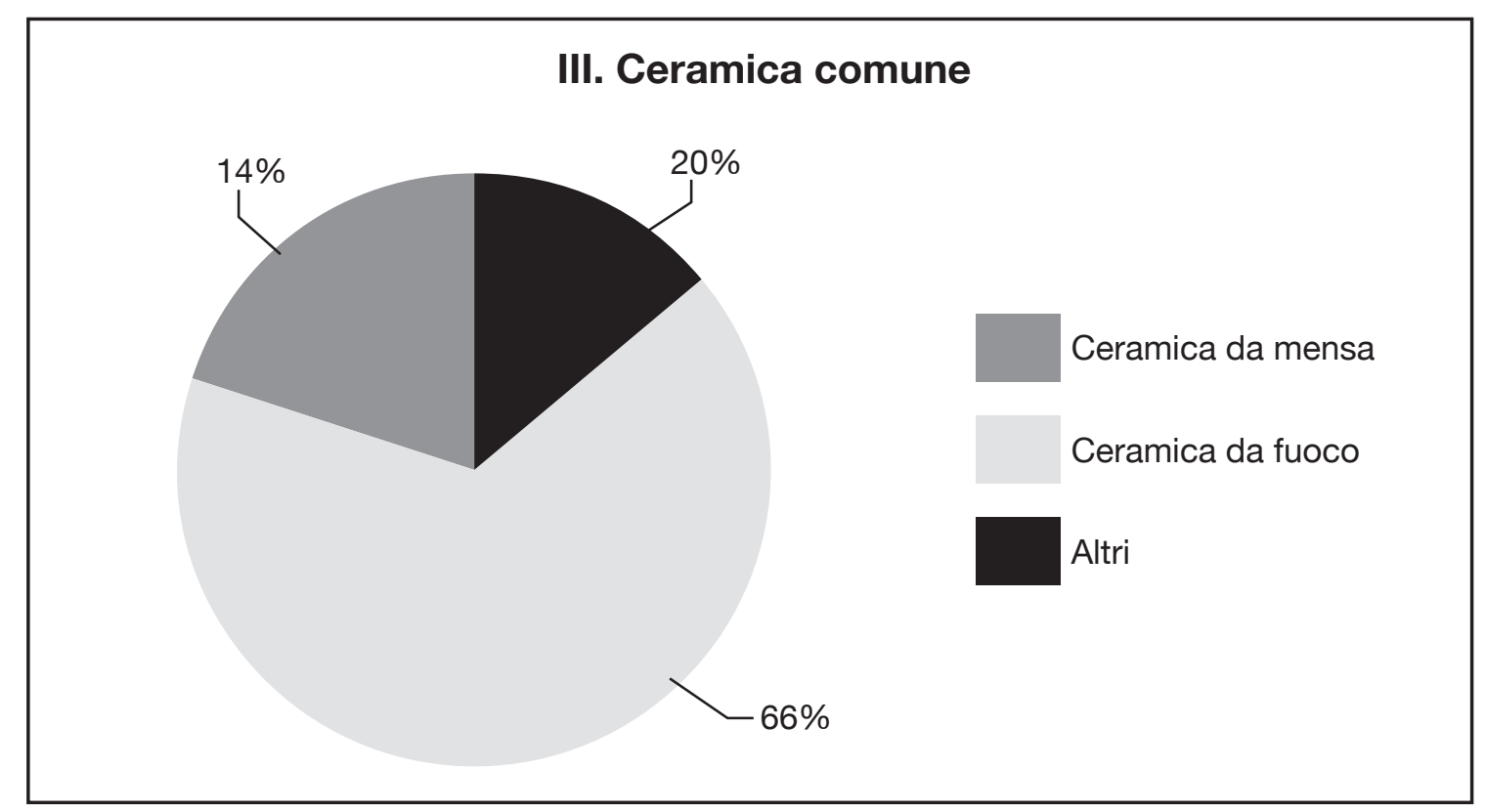

Fig. 31. Tipi della ceramica comune rinvenuta nel pozzo nel taglio g-4. 
prodotti cosmetici o medicinali (Fig. 28). Alcuni pezzi possono essere attribuiti al gruppo dei turiboli che, in un senso più vasto, potevano essere identificati come vasi che servivano a scopi di culto.

Entro l'ambito della tipologia della ceramica comune la proporzione della ceramica da mensa e da fuoco è del 2:5 (Fig. 30). La quantità dei vasi potori (tazze e coppe) è la metà di quelle delle brocche e olpai; d'altra parte al tipo di vasi potori si può aggiungere i tipi di ceramica fine, inquadrabili nel gruppo delle ceramiche a pareti sottili, ma anche se ai $36 \mathrm{fr}$. di vasi potori si aggiungono i 14 vasi di ceramica a pareti sottili, la quantità delle brocche continua a essere maggiore.

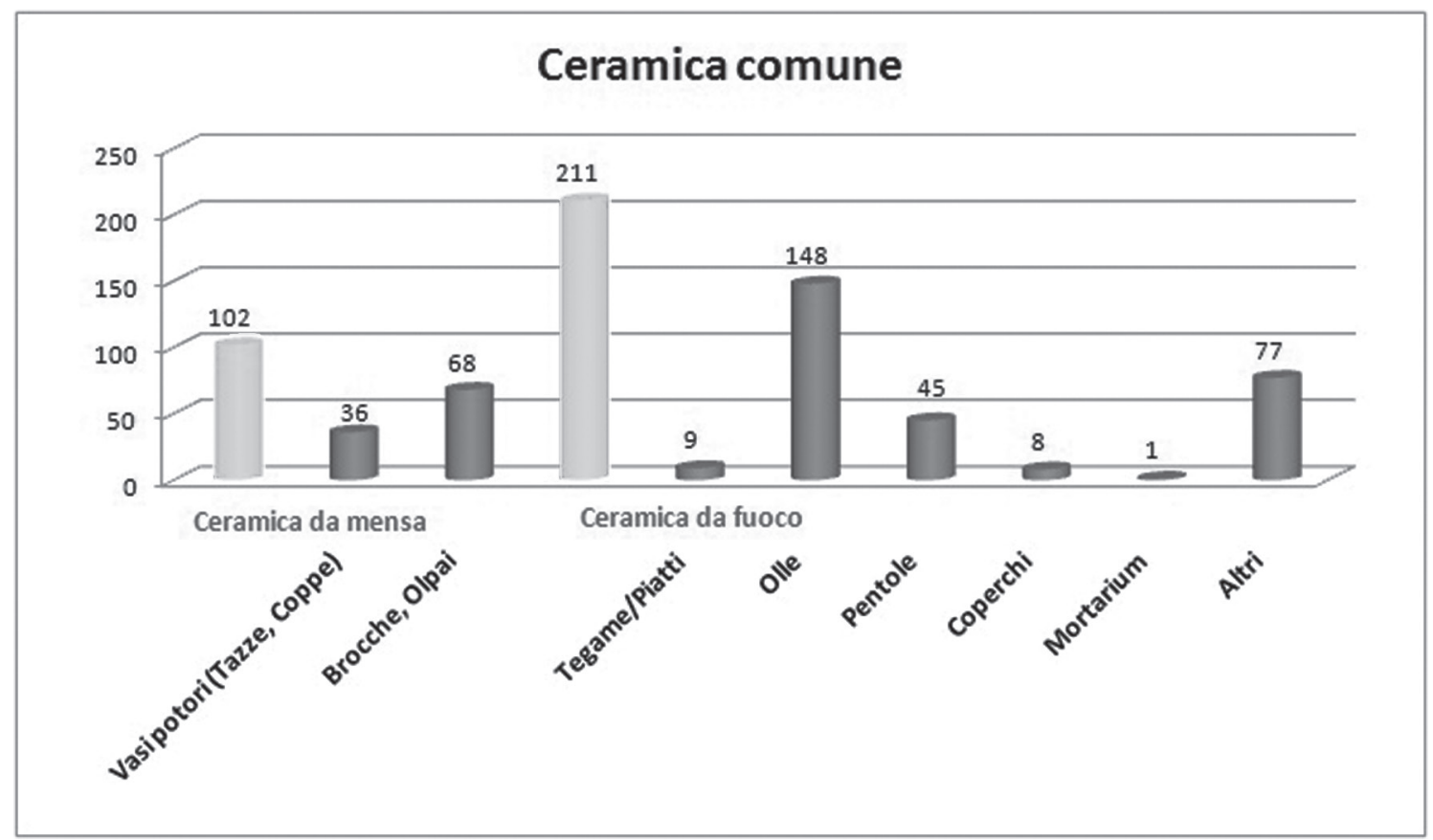

Fig. 32. Tipi della ceramica rinvenuta nel pozzo nel taglio g-4

Entro la categoria della ceramica da fuoco la quantità delle olle è la maggiore (148 frammenti), seguita dalle pentole (45 pezzi), e poi dai tegami (9 esemplari). Oltre ai pochi coperchi si aggiunge a questa tipologia anche un mortarium. Non si è potuto definire la forma di circa 77 frammenti, la maggior parte probabilmente deve essere ceramica da fuoco (Fig. 31). Il materiale della ceramica è argilla di color rosso giallastro o rosso tendente al marrone. Tra le pentole si osservano alcuni pezzi di argilla color ocra. Per due olle e una brocca abbiamo potuto stabilire che fossero state cotte in atmosfera riducente, l'orlo di quest'ultime è grigio e marrone grigiastro. Molte pentole hanno le pareti laterali annerite o grigie per via della cottura, ed è frequente anche l'orlo annerito (Fig. 32).

Nel riempimento della cisterna scavata nel centro del cortile settentrionale e dal canale che vi conduce, sono venuti alla luce una sigillata africana di tipo A 1, 9 vasi di ceramica a pareti sottili, 5 anfore, 153 ceramiche comuni, un dolium e una lucerna (Fig. 33). I frammenti delle anfore, entro i limiti creati dalla difficoltà di definire i pezzettini piccolissimi, possono essere inquadrati nella tipologia Dressel 2-4. Ciò significa che, solo uno dei 168 vasi può essere ritenuto sicuramente merce derivante da importazione.

La cosiddetta ceramica fine costituisce circa il $10 \%$ di questo materiale che, è in grandi linee, conforme al materiale rinvenuto nel taglio g-4. La differenza fra i due contesti si riferisce alla composizione delle sigillate, infatti, mentre nel materiale del pozzo di S si trovano varie sigillate italiche, in quello della cisterna $\mathrm{N}$ nessuna. Oltre alle sigillate dell'Africa Settentrionale si può inquadrare al tipo di ceramica fine la ceramica a pareti sottili, nonché la ceramica rivestita (Fig. 34). Sui reperti di 4 brocche e 2 tegami abbiamo trovato un rivestimento rosso o marrone rossastro. Le pentole che imitano forme nord-africane e certi tipi di tegami del materiale derivante dalla cisterna, 


\section{Ceramica per classe}

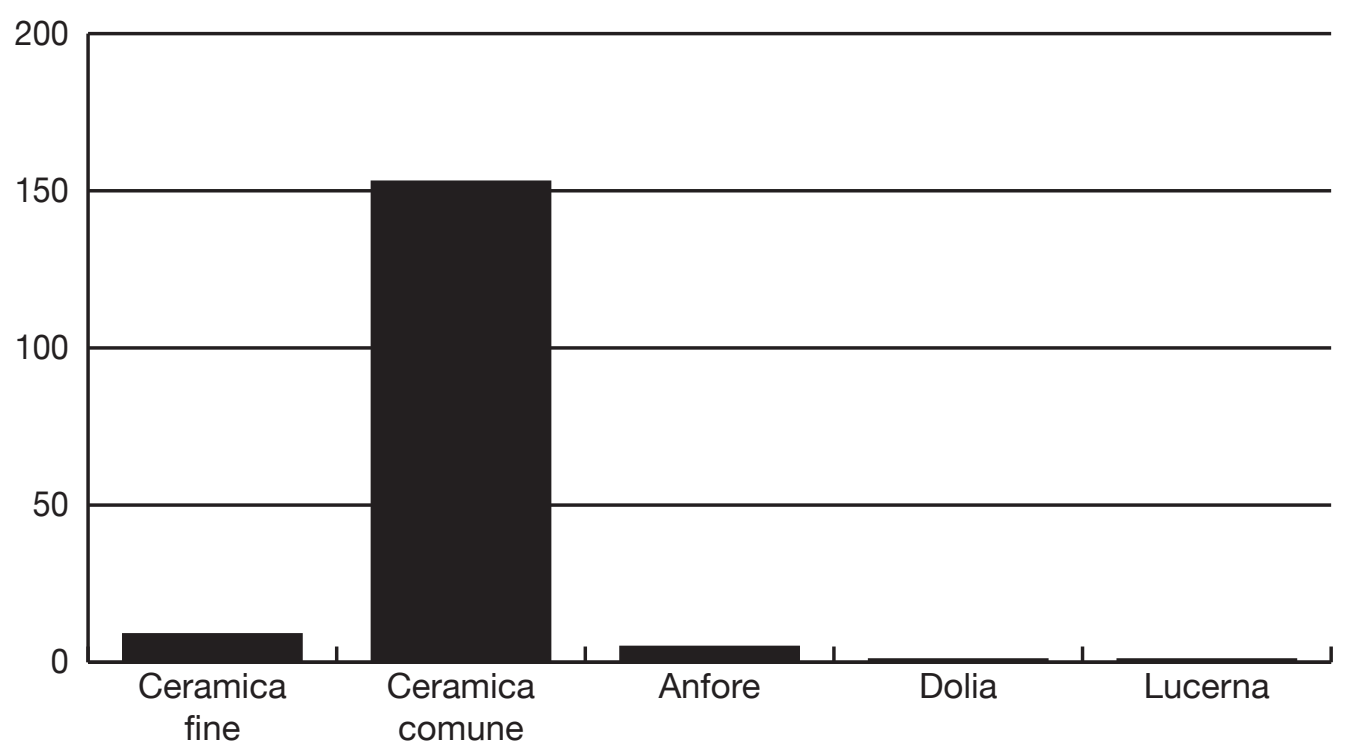

Fig. 33. Distribuzione percentuale della ceramica fine rinvenuta nella cisternae canale ubicati nel centro del cortile settentrionale

\section{Ceramica fine}

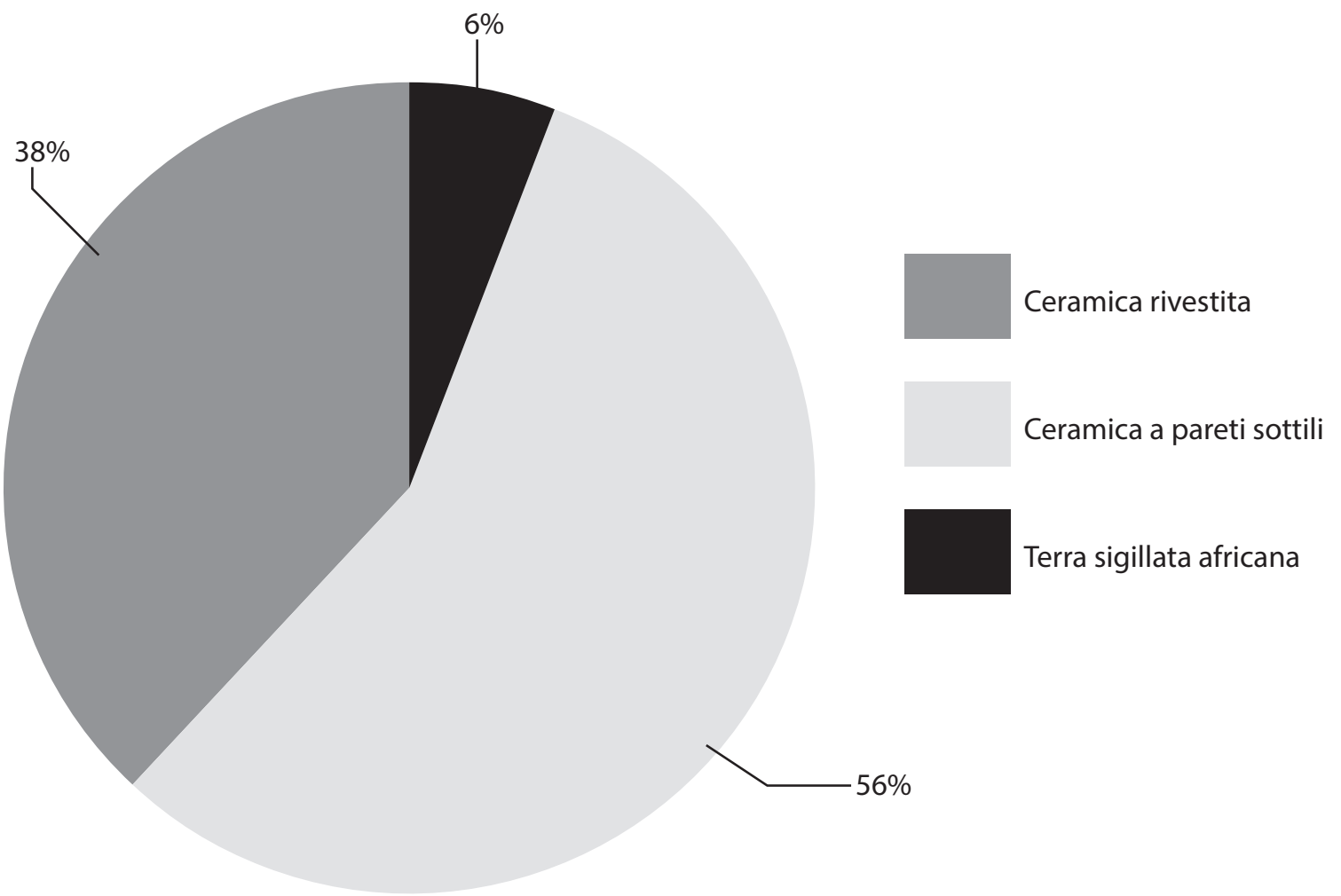

Fig. 34. Distribuzione della ceramica comune rinvenuta nella cisternae canale ubicati nel centro del cortile settentrionale 


\section{Ceramica comune}

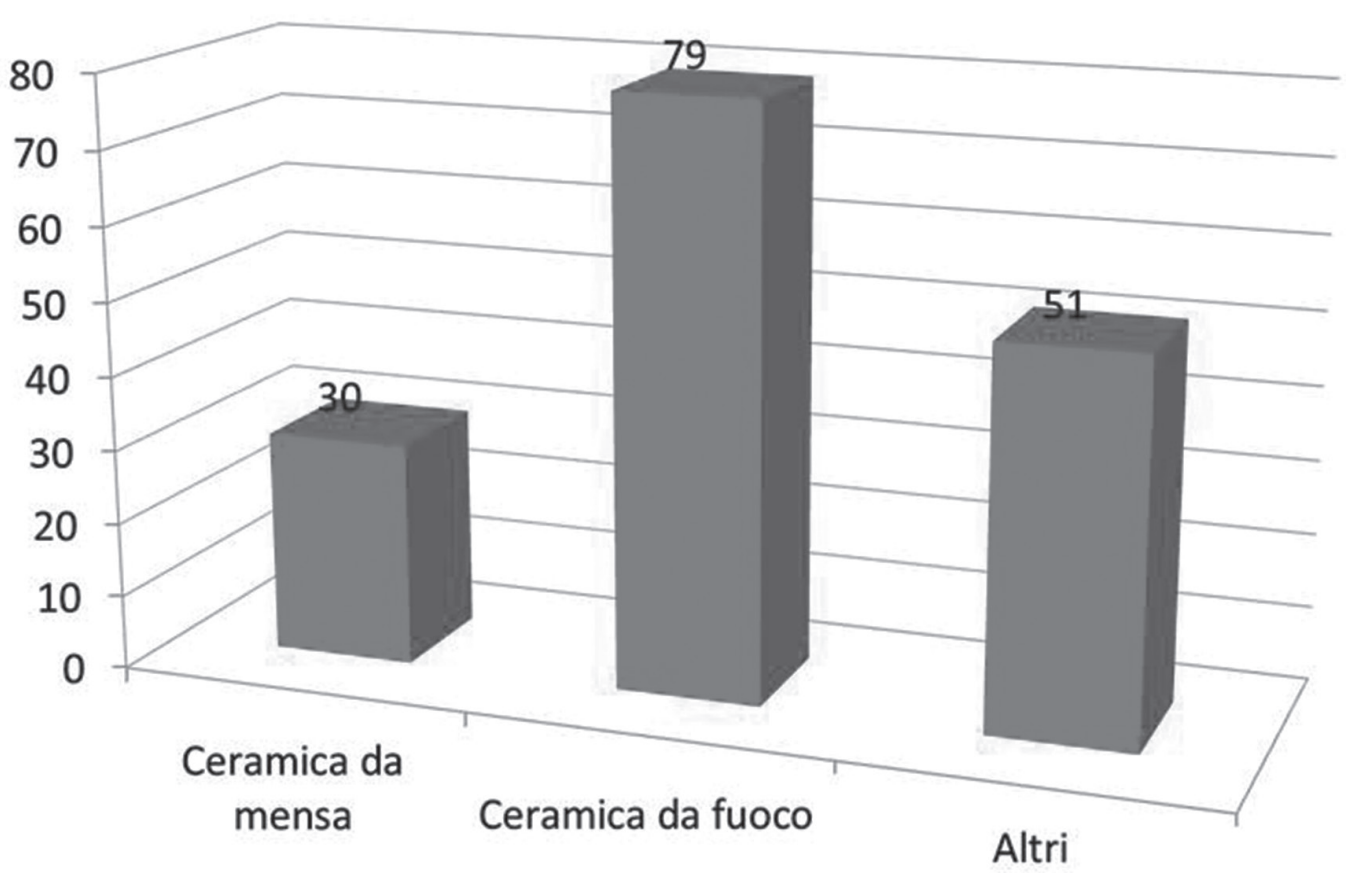

Fig. 35. Tipi della ceramica comune rinvenuta nella cisterna canale ubicati nel centro del cortile settentrionale

\section{Ceramica comune}

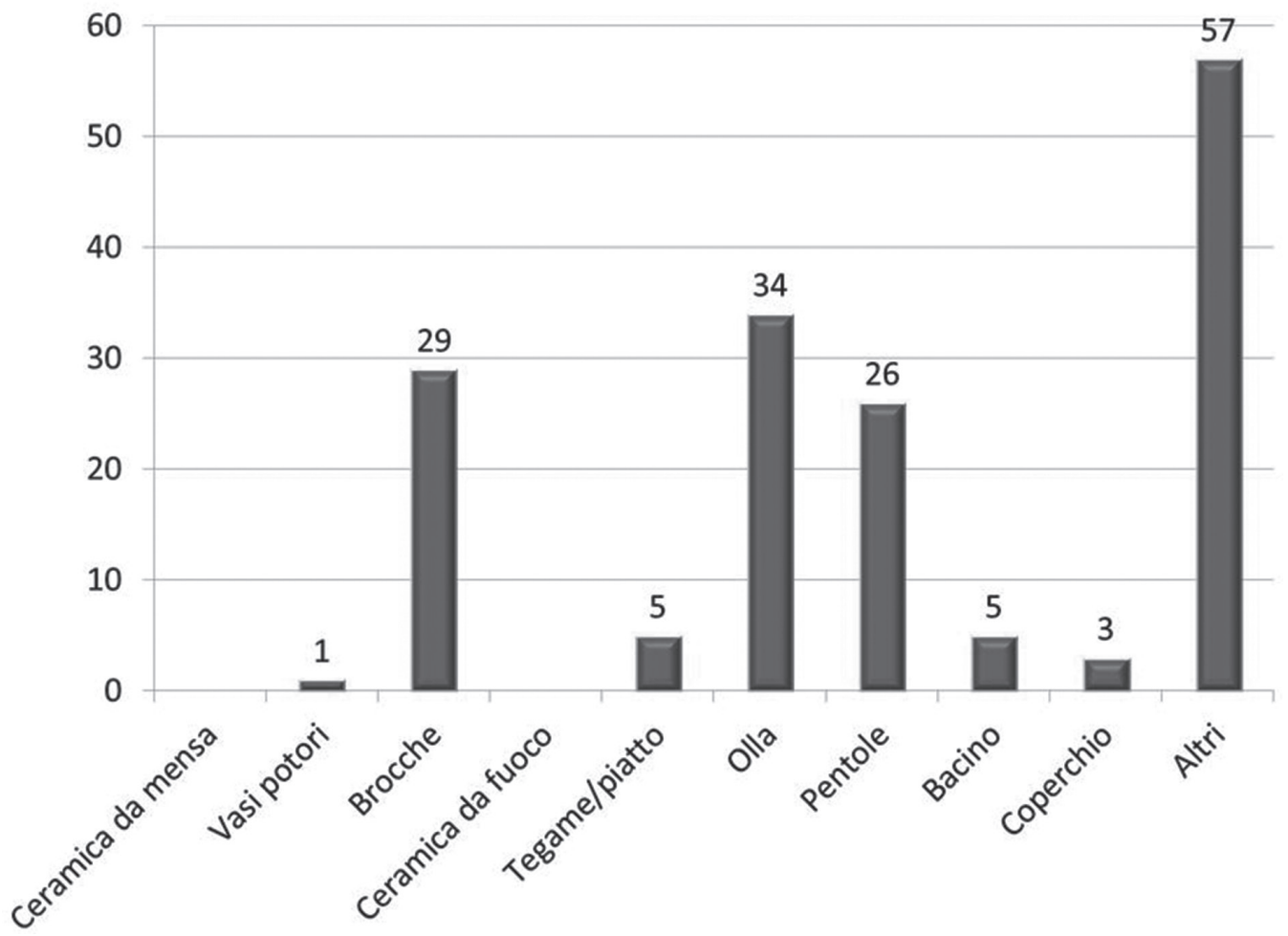

Fig. 36. Tipi della ceramica comune rinvenuta nella cisterna e canale conducente alla cisterna ubicati nel centro del cortile settentrionale. 
possono essere datati alla seconda metà del II sec., eventualmente al III sec. mentre gli altri vasi possono essere datati a un periodo che va dall'età flavia fino al II sec., altri invece non sono affatto databili. Un bacino diverso dagli altri tipi anche in base ai connotati qualificativi, risale a un tipo antico, di tradizioni ellenistiche proveniente dall'epoca tardo-repubblicana. Entro l'ambito della ceramica comune, il numero dei vasi da fuoco è più del doppio di quelli appartenenti alla categoria(Fig. 35-36) di ceramica da mensa (la proporzione dei due tipi è di 87:30) Il vasellame indicato con altra denominazione, sempreché la funzione sia definibile, nonostante la frammentarietà, probabilmente è inquadrabile al tipo della ceramica da fuoco. Tra i tipi della ceramica da mensa prevalgono le brocche (29), nel nostro materiale, oltre ai vasi a pareti sottili, abbiamo solo un frammento appartenente a un vaso potorio. È impressionante il basso numero di frammenti di tegami entro la ceramica da fuoco (34 pezzi), mentre nel materiale del pozzo S. ne abbiamo rinvenuto quattro volte tanto. In questo contesto, invece, sono venuti alla luce molti bacini (5), tipo questo che, manca completamente dall'altro contesto. La proporzione delle altre tipologie è pressappoco la medesima di quella osservata nell'altro contesto, è da notare però che, in quest'ultimo è stato rinvenuto circa il doppio di fr. di vasi, rispetto al primo. Il materiale anche in questo caso è rosso giallastro, rosso tendente al marrone, e raramente ocra-biancastro. L'argilla usata per la fabbricazione delle pentole è talvolta micacea e sulla maggior parte si trovano macchie annerite da combustione. Diverse olle si sono pure annerite in seguito all'uso, mentre l'argilla del bacino di forma peculiare è marrone scura con dentro inclusioni di calce.

Nello strato d'incendio del vano situato nella parte meridionale della villa più antica sono stati rinvenuti 132 frammenti di vasi, di cui solo l'1,5\% era merce importata (Fig. 37). Oltre alla sigillata di La Graufesenque probabilmente apparteneva a questo gruppo un'anfora, sempreché la forma di questa sia veramente Dressel 7-11. La proporzione della ceramica fine in questo sito raggiunge appena il 5\%, oltre al fr. di una sigillata di origine italica, a questo gruppo possono essere inquadrati fr. di ceramica a pareti sottili. (Fig. 37) Qui pure sono venuti alla luce 3 fr. di ceramica con rivestimento rossastro. Lo s pettro della ceramica rinvenuta nello strato d'incendio differisce perfino da quello del materiale del pozzo g-4, benché ambedue contenessero reperti provenienti dall'età flavia e del regno di Traiano. Nello strato d'incendio abbiamo rinvenuto molti fr. di anfore (18 pz.), ma di questi solo due-tre poterono essere collegati a forme concrete. La proporzione tra la ceramica da mensa e la ceramica da fuoco (Fig. 38-39) differisce da quella osservata negli altri contesti, giacché il numero di quest'ultima supera di poco quella della ceramica da mensa (40:56). In questo strato sono venuti alla luce due dolium e due lucerne; tra quest'ultime è presente anche il cosiddetto tipo di Vogelkopflampe. La maggior parte della ceramica da mensa recuperata sono

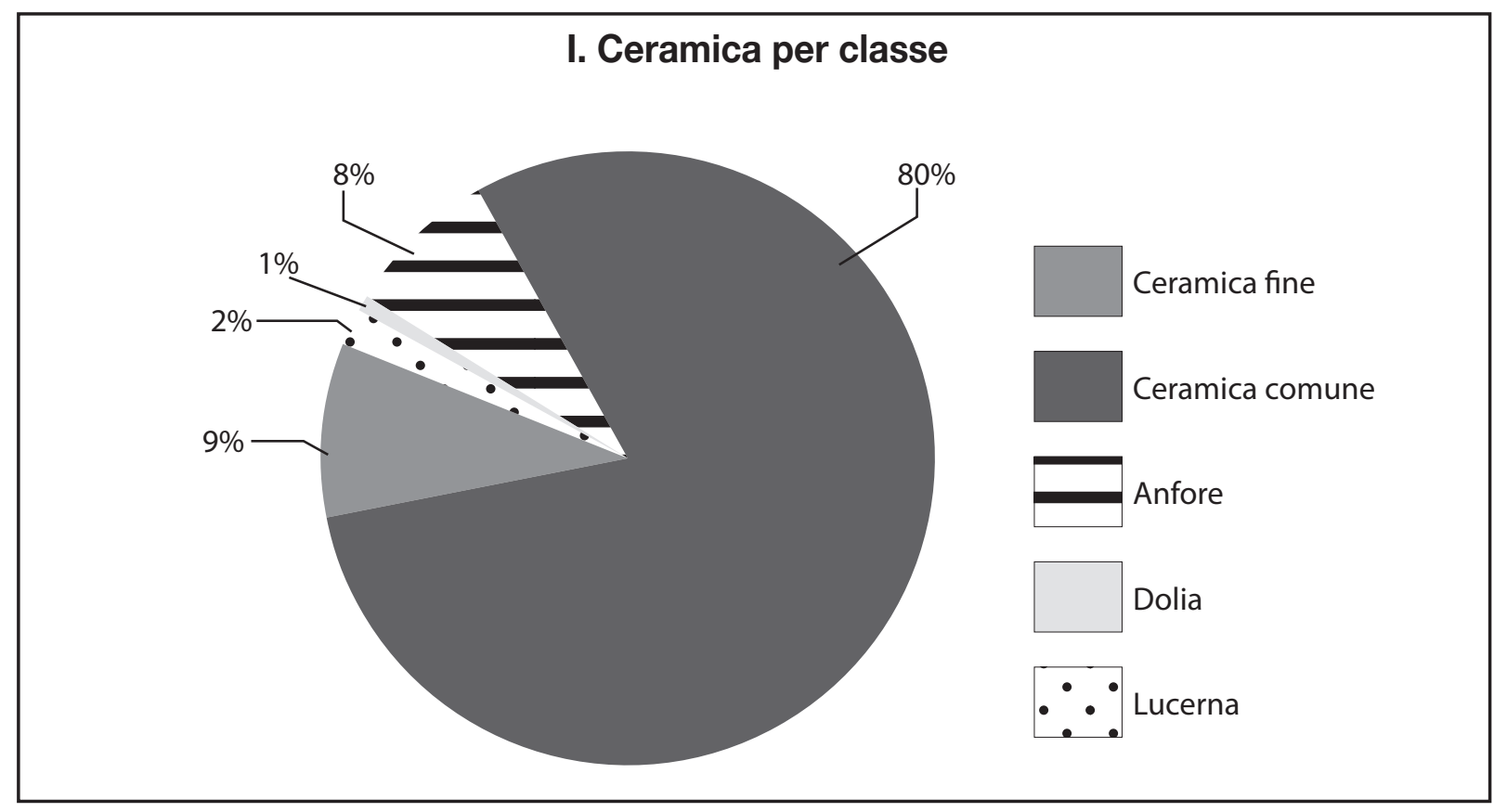

Fig. 37. Distribuzione percentuale della ceramica rinvenuta nello strato d'incendio sito nella parte meridionale della villa più antica. 


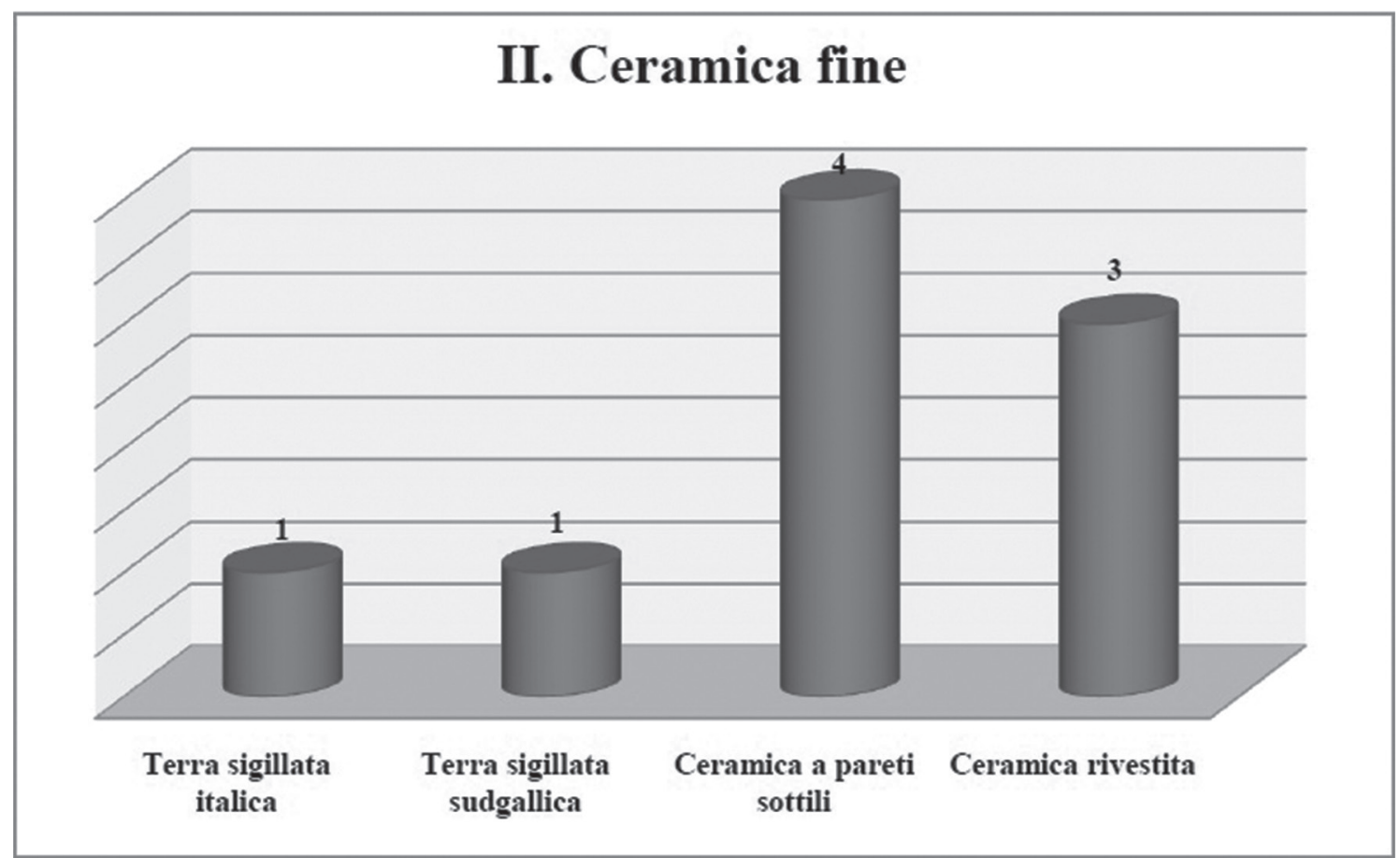

Fig. 38. Tipi della ceramica fine rinvenuti nello strato d'incendio nella parte meridionale della villa più antica.

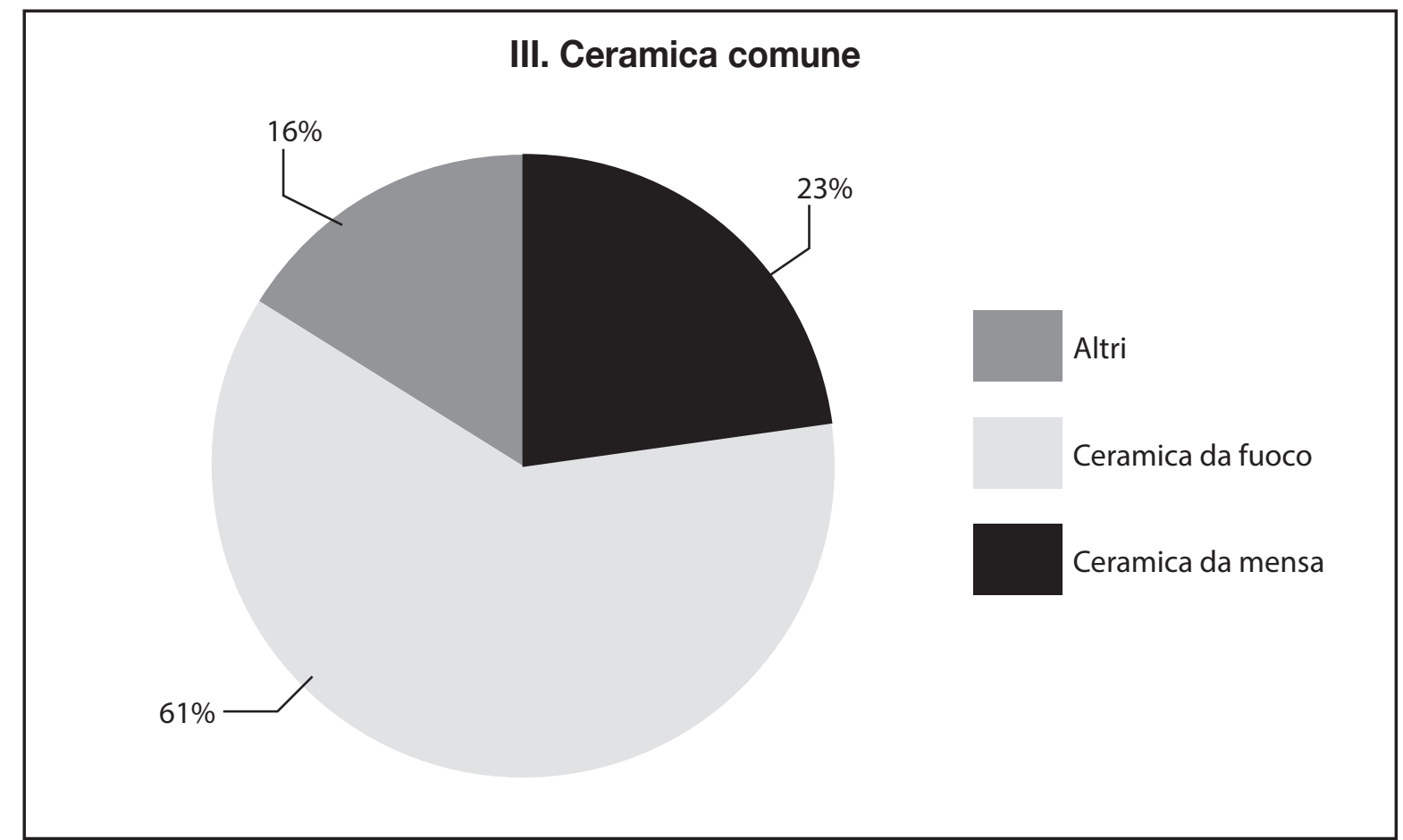

Fig. 39. Distribuzione percentuale della ceramica comune rinvenuta nello strato d'incendio nella parte meridionale della villa più antica. 


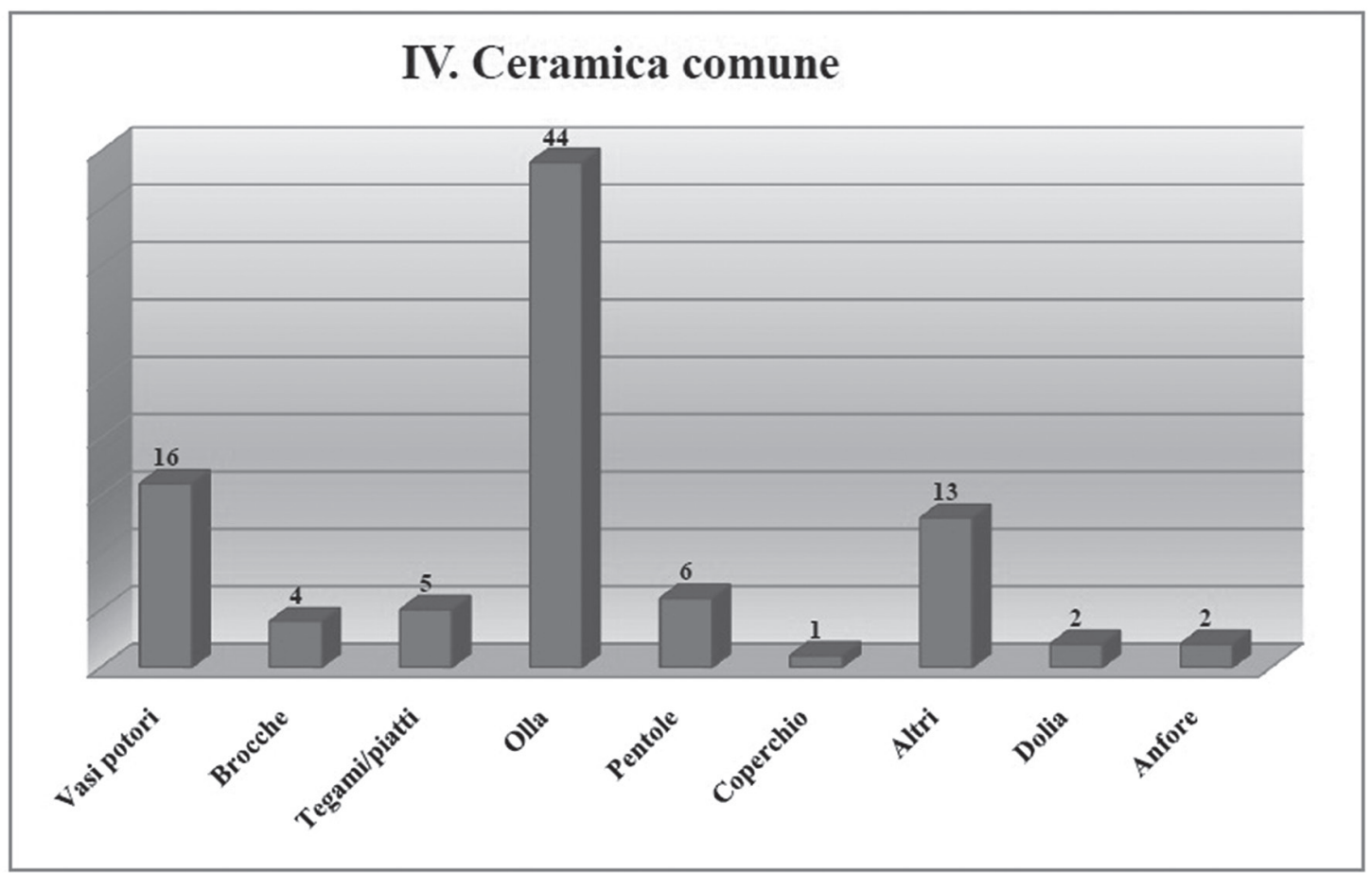

Fig. 40. Tipi della ceramica comune rinvenuti nello strato d'incendio nella parte meridionale della villa più antica.

brocche, il numero delle tazze è minore. I tipi di ceramica da fuoco sono per lo più olle (44 pz.) (Fig. 40), il numero delle pentole (6) e dei tegami (5) è molto minore e si rinvenne in tutto solo un coperchio, mentre in questo strato non trovammo nessun bacino, mortarium o turibulum. Il materiale dei vasi anche qui è prevalentemente rosso giallastro o rosso tendente al marrone, con frequenti inclusioni di calce e particelle nere sparse.

Oltre ai tre contesti sigillati e al materiale degli stessi, abbiamo pubblicato anche alcuni tipi caratteristici aventi soprattutto un certo rilievo tipologico, provenienti da siti in cui non si poteva effettuare osservazioni (per es. macerie e detriti, humus arato). Nell'ultimo ambiente verso $\mathrm{N}$ con pavimento di coccio pesto della serie di locali occidentali della parte centrale sono venuti alla luce olpai, una brocca di tipo Olcese 2, un tegame con rivestimento rosso, nonché un'olla di forma Olcese 6/7 che si poterono ricostruire quasi completamente. Si suppone che questi vasi si conservarono quasi intatti perché probabilmente rimasero in uso fino all'ultimo periodo della villa, fino alla seconda metà del III sec. Le tipologie menzionate, in base agli accertamenti stratigrafici eseguiti a Ostia, nelle Terme del Nuotatore, possono essere datate al II sec. fino all'inizio del III sec. L'ipotesi che fossero rimaste in uso in età più tarda è attestato per l'appunto dalle condizioni di rinvenimento osservate nella villa di San Potito. Prima abbiamo potuto osservare lo stesso fenomeno in base alla ceramica comune africana, infatti, nel ambiente XXIX, a O dalla II entrata principale della villa furono rinvenuti un piatto/coperchio, nonché una ceramica polita a strisce. Questi vasi probabilmente furono pure usati all'epoca della rovina della villa e per questa ragione si riuscì a ricostruirli da numerosi frammenti. ${ }^{349}$ La vasta commercializzazione della ceramica comune Nord-Africana è attestata nel periodo che va dalla seconda metà del II sec. al V. Per queste tipologie non si può eseguire una datazione più precisa. Si ipotizza che giungessero in Italia in accompagnamento delle forniture di cereali e di olio; è praticamente escluso che negli Abruzzi venisse importato separatamente questo tipo di vasellame molto semplice dall'Africa del Nord. 
Grazie per il sostegno ricevuto dalla Fondazione Nazionale per la Ricerca Scientifica (Hungarian Scientific Research Fund - abbreviazione OTKA progetto $\mathrm{N}^{\circ}$ K104717) che, ha reso possibile l'effettuazione di NAA (Analisi per Attivazione Neutronica) e di indagini chimiche e geologiche, eseguite dalla dott.ssa Márta Balla e il professore dr. György Szakmány. Si ringrazia Lavinia Sándor per la traduzione del testo in lingua italiana. Siamo grati a Zsuzsanna Ba e Magdolna Eber per la grafica, il disegno e la redazione delle tavole. Le tavole Excel sono opera di Ibolya Szücs e Alexandra Kiss. Per le foto esprimiamo i nostri ringraziamenti a Bernadette Purtzl e al Dr. Ferenc Redő.

\section{BIBLIOGRAFIA}

\section{Albintimilium \\ ANGELETTI et alii 2000}

Angera 1985

ANNECHINO 1977

ANNESE et alii 2000

BAILEY 1980

BATs 1988

BELTRÁN 1978

BERGAMINI 1973

Bonnet-Batigne Vallet 2001

BÓNIS 1942

BRECCIAROLI TABORELLI 2010

BRUCKNER 1965

BRUNO 2005

BUCHI 1975

Cagano 1994

CARbonara-Messineo 1991-1992

Carbonara-Messineo 1991

CARRARA 1998

Castel Giubileo v.

Cavalieri Manesse 1975

Ceramiche in Lombardia 1998

Cerulli Irelli 1977

Ciotola 2012
$=$ v. LAMBOGLIA 1950

= C. Angeletti-L. Formicone-D. Formicone: La villa romana a Grasciano sec.I a.C- II.d.C. Mostra archeologica, Museo Civico “G.Romuladi”. Notaresco 2000.

= Angera romana. Scavi nella necropoli 1970-1979. (a cura di G. Sena Chiesa). Roma 1985

= M. AnNeChino: Suppelletile fittile da cucina di Pompei. In: L'instrumentum domesticum di Ercolano e Pompei nella prima età imperiale (a cura di A. Carandini). Roma 1977, 105-120.

= C. Annese-G. De Felice-M. Turchiano: Ceramiche della prima e media età imperiali di riempimenti delle latrine dalla domus A. Ordona X (ed. G. Volpe). Bari 2000, 251-265.

= D. M. BAILEY: Catalogue of the Lamps of the British Museum. II. Roman lamps made in Italy. London 1980.

= M. BATs: Vaiselle et alimentation à Olbie de Provence (v. 350-v. 50 av.J-C.). Modèles cultureles et catégories céramique. Revue archéologique de Narbonnaise suppl.18. Paris 1988, 1-166.

= M. BELTRÁn LloRIS: Ceramica romana, tipologia y clasificacion. Zaragoza 1978.

= M. BERGAmini: La ceramica romana. Romagna antica. Quaderni degli Studi Romagnoli 8. Faenza 1973

= C. Bonnet-C. Batigne VALlet: Contribution à l'étude des céramiques gríses kaolinitiques voconces d'après le mobilier issu des fouilles de tracè drõ̃mais du TVG Mediterranée. Acta RCRF 37 (2001) 73-88

= É. BóNIS: Die kaiserzeitliche Keramik von Pannonien I. DissPann ser.II.20. Budapest 1942

= L. Brecciaroli TABorelli: Ceramica a pareti sottili. In: Oro, pane e scrittura. Memorie di una comunità inter Vercellas et Eporediam (a cura di L. Brecciaroli Taborelli) Studi e ricerche sulla Gallia Cisalpina. Roma 2011

= A. BRUCKNER: Küchengeschirr aus der Casa del Fauno in Pompeji. Acta RCRF 7 (1965) 7-14

= B. BRUNO: Le anfore di trasporto.in: D. Gandolfi: La ceramica e i materiali di età romana. Classi, produzioni, commerci e consumi. Bordighera 2005, 368-369

= E. BuchI: Lucerne dem Museo Nazionale di Aquileia I. Lucerne romane con marchio di fabbrica. Aquileia 1975

= A. Cagano: Un contesto ceramico di età imperiale. Scavi in Palazzo Calissario di Alba. Quaderni della Soprintendenza archeologica di Piemonte 12 (1994) 107-126

= A. Carbonara-G. Messineo: La Celsa (circ.XX). Bollettino della Commissione Archeologica Comunale di Roma 1991-1992, 179-199

= A. Carbonara-G. Messineo: Ceramica delle fornace dalla Celsa.in: G. Messineo: La via Flaminia da Porta del Popolo a Malborghetto. Roma 1991

= M. CARRARA: Prima porta. La collina della torre. Bollettino della Commissione Archeologica Comunale di Roma 99 (1998) 381-391

= QUILICI 1976

= G. CAVAlieri Manesse: Il capitolium e la decorazione architettonica romana di Brescia. Atti del convegno internazional per il XIX centenario della dedicazione del capitolium e il $150^{\circ}$ anniversario della scoperta. II. Brescia 1975, 53-66

= Ceramiche in Lombardia tra II sec. a.C. e VII sec. d.C. Raccolta dei dati inediti (a cura di G. Olcese). Documenti di archeologia 16. Milano 1998

= G. Cerulli Irelli: Officine di lucerne fittili a Pompei. Quaderni di cultura materiale I. L'instrumentum domesticum di Ercolano e Pompei nella prima metà mperiale. Roma 1977

= A. Ciotola: Ceramica con ingobbio rosso. In: Lacus Juturnae II. Materiali-Acta Instituti Romani Finnlandiae 38 (2012) 203-207 
CIPRIANO-DE FABRIZIO 1996

Coletti-Pavolini 1996

CORTI 2012

D'AMBROSIO-BORRIELLO 2001

DE BonIs et alii 1996

De CARo 1975

De Caro 1994

Deneauvue 1969

Di GiovanNi 1996

DOBRZANSKA 2000

DunCAN 1964

DYSON 1976

FABBRI 2008

FAGA 2008

FAGA 2011

GABLER 2011

GABLER 2012

GABLER-BEnCZE 1998

GABLER-REDŐ 1986

GABLER-REDŐ 2008

GALLO 1994

GAMBERO 1999

GASPARETTI 1996

GERVASINI 2005

GORI-PIERINI 2001

GOUDINEAU 1970
= M. T. CIPRIANO-S. DE FABRIZIO: Benevento. Il quartiere ceramico de Cellarulo: prime osservazioni sulla tipologia ceramica. In: Les céramiques communes de Campagne et de Narbonnaise (I er siécle avant J-C-II e siécle apres J-C.) Actes des Journées d'étude organisée par la Centre Jean Bérard et la Soprintendenza archeologica per le province di Napoli e Caserta (sous la direction de M.Bats). Naples 1996, 203-221

= M. Voletti- C. Pavolini: Ceramica comune da Ostia. In: Les céramiques communes de Campanie et de Narbonnaise. Actes des Journées d'étude organisée par le Centre Jean Bérard et la Soprintendenza archeologica per le Province di Napoli e Caserta (sous la direction M. Bats). Naples 1996, 391-419

$=$ C. CORTI: Ceramiche a rivestimento rosso della media e tarda età imperiale in: Insediamento romano della tesa di Mirandolo (MO). Ricognizioni e scavi 1930-2011. Quaderni di Archeologia dell'Emilia Romagna 30 (a cura di M. Calzolari- M.Foran)-Firenze 2012, 102-108

= A. D'Ambrosio-M. Borriello: Arule e bruciaprofumi da Pompei. Studi della Soprintendenza archeologica di Pompei 3. Napoli 2001

= A. De Bonis-L. Cavanassa-C. Grifa-A. Langella-V. Morro: Le ceramiche comuni di Cuma. In: Les céramiques communes de Campanie et de Narbonnaise. Actes des Journées d'étude organisées par le Centre Jean Bérard et la Soprintendenza Archeologica per le province di Napoli e Caserta (sous la direction M. Bats) Naples 1996, 309-330

= S. DE CARo: Le lucerne dell'officina LVC. Rendiconti della Accademia di Archeologia di Napoli 49 (1974) 107-134

$=$ S. DE CARO: La villa rustica in località Villa Regina a Boscoreale. Roma 1994

= J. Deneauvue: Lampes de Carthage. Paris 1969

$=$ V. Di GiovanNi: Produzione e consumo de la ceramica da cucina nella Campania romana. In: Les céramiques communes de Campanie et de Narbonnaise. Actes des Journées d'étude organisée par le Centre Jeand Bérard et la Soprintendenza archeologica per le province di Napoli e Caserta (sous la direction M.Bats). Naples 1996, 65-103

$=$ H. DobrZANSKa: Early Roman pottery imports from the Northern Pontic area and Asia minor found in Poland. Hellenistic traditions and Roman design. Acta RCRF 36 (2000) 337-340

= G. C. Duncan: A Roman Pottery near Sutri. Papers of the British School at Rome 32.n.s. 19 (1964) 38-88

= S. J.Dyson: Cosa. The Utilitarian Pottery. Mem.of American Academy Rome 33. Roma 1976

= F. FABBRI: Ceramic basins from the "Kilns of Vingone" in Scandicci (Florence, Italy). Aegean shapes in the pottery production in the territory of Roman Florentia. Acta RCRF 40 (2008) 339-345

4 I. FAGA: Ceramica a pareti sottili del complesso archeologico di San Lorenzo Maggiore (Napoli). Acta RCRF 41 (2008) 189-198

= I. FAGA: La ceramica a pareti sottili.in: Scoppieto II. I materiali (a cura di M. Bergamini). Firenze 2011, 127-227

= D. GABLER: Ceramica fine rinvenuta nella villa di San Potito (AQ). ActaArchHung 62 (2011) 91-124

= D. GABLER: Sigillate galliche meridionali ad Emona e in Italia. In: Emona between Aquileia e Pannonia (ed. I. Lazar- B. Županek). Koper 2012, 49-59

= D. GABLER-Á. BENCZE: Reperti vitrei della villa romana di San Potito di Ovindoli. Acta ArchHung 50 (1998) 91-197

= D. GABLER-F. REDŐ: Gli scavi nella villa romana a San Potito di Ovindoli (L'Aquila) 1983-1984. ActaArchHung 38 (1986) 41-87

= D. GABLER-F. Redỏ: Lo svolgimento strutturale della villa romana di San Potito. In: Richerche archeologiche a San Potito di Ovindoli e le aree limitrofe nell'antichità e nell'alto medioevo. (a cura di D. Gabler-F. Redö). L’Aquila 2008, 41-70

= A. Gallo: La casa di Lucio Elvio Severo a Pompei. Accademia di Archeologia, Lettere e Belle Arti. Monumenti IX. Napoli 1994

= L. GAMBERO: La Liguria costiera tra III e I sec.a.C. Mantova 1999

= G. GASPARETTI: Produzione e consumo della ceramica comune da mensa e dispensa nella Campania romana. In: Les céramiques communes de Campanie et de Narbonnaise ( 1 er s.av.J-C.-II e s. ap.J-C.). La vaiselle di cuisine et de table. Actes des Journées d'étude arquisées par le Centre Jean Bérard et la Soprintendenza Archeologica per le Province di Napoli e Caserta. Naples 27-28 mai 1994 sous la dirction M. Bats. Naples 1996, 19-63

= C. Gervasini: La ceramica a pareti sottili. In: D. Gandolfi: La ceramica e i materiali di età romana. Classi, produzione, commerci e consumi. Bordighera 2005, 279-310

= B. GoRI-T. PIERINI: La ceramica comune di argilla figulina. In: Gravisca. Scavi nel santuario greco. Bari 2001

$=\mathrm{CH}$. Goudineau: Note sur la céramique à engobe interne rouge-pompeien. (pompeianisch roten Platte). MEFR 82 (1970) 159-196 
HARSÁNYI 2006

HÁRSHEGYI 2008

HAYES 1973

HAYES 1983

HEERES 1972

HILGERS 1969

JONCHERAY 1974

KENRICK 1985

KOLLÁTH 2011

LAMBOGLIA 1950

LAMBOGLIA 1952

LARESE 1983

LEIBUNDGUT 1977

Luni I

Luni II

Maioli 1972-1973

MASSEROLI 1997

Marabini Moevs 1973

Martin-De Sena 2003

MARTIN-KILCHER 1994

MARUCCI 2006

MASSARI 1979

MAYET 1975

MazZeO SARACINO 1977

MeNCHELLi 2003

MenCHelli 2005

MENCHELli 2012

MENCHELli et alii 2005

MENCHELLI-PASQUinUCCI 2012

MENZEL 1954

MihaEsCu-BîRLiBA 1996

OLCESE 1993

OLCESE 1996
= E. HARSÁNYI: A füstölőtálak és a füstölés szokása a császárkorban. Antik tanulmányok 50 (2006) 57-73

= P. HÁrshegYi: Le anfore della villa romana di San Potito. In: Ricerche archeologiche a San Potito di Ovindoli e le aree limitrofe nell'antichità e nell'alto medioevo (a cura di D. GABLER-F. REDÖ). L'Aquila 2008, 133-177

= J. W. HAYES: The Roman Pottery from the South Stoa at Corinth. Hesperia 42 (1973) 416-470

= J. W. Hayes: The Villa Dionysos Excavations, Knossos. The Pottery. Annual of the British School, Athens 78 (1983) 97-169

= G. HeERES: Die römischen Bildlampen der Berliner Antiken-Sammlung. Berlin 1972

= W. HiLGERS: Lateinische Gefäßnamen. Bezeichnungen, Funktion und Form römischer Gefäße nach den antiken Schriftquellen. Beih. Bonner Jb 31. Düsseldorf 1969

= J.-P. Joncheray: Etude de l'épave Dramont “D” dite “de Pelvis". CahierArchSubaqu 3 (1974) 21-44

$=$ PH. KenRICK: The fine pottery. Excavations at Sidi Khrebish Benghazi (Berenice) III.1- suppl. to Libya Antiqua. Tripoli 1985

= Á. Kolláth: Le ceramiche medievali di San Potito. ActaArchHung 62 (2011) 125-146

= N. LAmbogliA: Gli scavi di Albintimilium e la cronologia della ceramica romana. Campagne di scavo 1938-1940. Bordighera 1950 (ristampa 1979)

= N. Lamboglia: La nave romana di Albegna. Storia e vicende della scoperta. RivStudi Liguri 18 (1964) 131-236

= A. LARESE: Le lucerne fittili e bronzee del Museo Concordiese di Portogruaro. Roma 1983

= A. LeIBundgut: Die römischen Lampen in der Schweiz. Eine kultur-und handelsgeschichtliche Studie. Bern 1977

= AAVV, Scavi di Luni. Relazione preliminare delle campagne di scavi 1970-1971 (a cura di A. Frova). Roma 1973-1974

= G. MASSARI-G. RATTI: Osservazioni sulla ceramica comune di Luni. In: Scavi di Luni II. Relazione delle campagne di Luni 1972-1974 (a cura di A. Frova). Roma 1977, 590-630

= M. G. MAIOLI: Vasi a pareti sottili grigie dal Ravennate. Acta RCRF 14-15 (1972-1973) 106-124

= S. MAsseroli: Ceramica a pareti sottili. Un pozzo e il suo contesto. Quaderni di ACME 29 (a cura di G. Sena Chiesa-S. Masseroli-T. Medici-M. Volonté). Milano 1997, 65-75

= M.t. Marabini Moevs: The thin walled Pottery from Cosa. (1948-1954) MemAmerican Academy Rome 32. Roma 1973, 283-301, 698-701, 762-767

= A. MARTin-E. De SENA: Ostia-Overview of the pottery. Acta RCRF 38 (2003) 43-49

= S. MARTIN-KILCHER: Die römischen Amphoren in Augst und Kaiseraugst. Ein Beitrag zur römischen Kulturgeschichte 2. Die Amphoren für Wein, Fischsauce, Südfrüchte (Gruppe 2-24) und Gesamtauswertung. Forschungen in Augst 7/2. Augst 1994

= A. MARUCCI: Foro transitorio. Lo scavo dei fori imperiali 1995-2000.I contesti ceramici. École française de Roma. (a cura di A. Santangeli Valenzani-R. Meneghini) Roma 2006

= G. MASSARI: Metodologia di ceramica applicata alla ceramica d'uso comune di Luni. Acta RCRF 19-20 (1979) 38-52

= F. MAYET: Les céramiques à parois fines dans la Péninsule Ibérique. Paris 1975

= L. MAZZeo SARAcino: Russi (Ravenna). Campagne di scavo 1971. NotSc 31 (1977) 5-156

$=\mathrm{S}$. Menchelli: Per una classificazione delle ceramiche comuni di età romana nell`Etruria settentrionale costiera. Acta RCRF 38 (2003) 279-286

= S. MenCHELli: Vasi comuni nella Tuscia settentrionale costiera. Aspetti regionali ed apporti mediterranei. Acta RCRF 39 (2005) 109-118

= S. MENCHELLI: 'L'ager Firmanus' dall' età tardo repubblicana alla conquista longobarda. I paesaggi piceni e romani nelle Marche meridionali. 2012

= S. Menchelli-M. Pasquinucci-I. Monacci-G. Picchi-P. Sangriso: Vada Volaterrana, scavi e ricerche 2004. Quadro del Laboratorio Universitario Voltereno 8 (2005) 217-225

= S. Menchelli-M. PAsQuinucci: Ceramiche con rivestimento rosso nella Tuscia settentrionale. Acta RCRF 42 (2012) 229-237

= H. MenZel: Antike Lampen im Römisch-Germanischen Zentralmuseum zu Mainz. Katalog des Römisch-Germanischen Zentralmuseums 15. Mainz 1954

= V. MiHAESCU BîRLiBA: Turibula: concerning the origin and the utilization of a pottery category from the Lower Danube. Acta RCRF 33 (1996) 97-102

= G. OLCESE: Le ceramiche comune di Albintimilium. Indagine archeologica e archeometrica. Quaderni del Dipartimento di Archeol. e Storia dell'arte Univ. Siena. Firenze 1993

= G. OLCESE: Ceramiche comuni di origine centro-meridionale tra il II. secolo a.C. e il I.d.C problemi aperti. L'evidenza dei reperti di Albintimilium. In: Les céramiques de Campanie et de Narbonnaise Ier a. av.J.C-IIe sec. ap.J.C. La vaiselle de cuisine et de table. Actes des Journées d'étude arquisées 
OLCESE 2003

Ostia I

Ostia II

Ostia III

Ostia IV

Ostia 1978

Ostia XIII

PALLECHI 2002

PANELla 1989

PATTERSON et alii 2003

PAVOLINI 1976-1977

PAVOLINI 2000

PEŇA 1987

Petru 1972

PLESNIČAR 1972

POHL-ZEVI 1970

PONGRÁCZ 1994

PUCCI 1975

PUCCI 1985

PUCCI 1992

QUILICI 1976

REYNOLDS et alii 2008

RICCI 1974

RICCI 1985

Rizzo 1998

ROBINSON 1959

SANTORO-BIANCHI-FABBRI 1997

SCHINDLER-KAUDELKA 1975

SCHINDLER-KAUDELKA 1998

SCHINDLER-KAUDELKA 2012 par le Centre Jean Bérard et la Soprintendenza Archeologica per le Province di Napoli e Caserta Naples 27-28 mai1994 (sous la direction M. Bats). Naples 1996, 421-445

= G. OLCESE: Ceramiche comuni a Roma e in area romana: produzione, circolazione e tecnologia (tarda età repubblicana-prima età imperiale (con contributi di: C. Coletti, E. G. Lorenzetti, M. Picon, G. Tassinari, G. Thierin Michael). Documenti di Archeologia 28. Mantova 2003

= AAVV: Ostia I. Le Terme del Nuotatore, scavo dell' ambiente IV. Studi miscellanei 13. Roma 1968

= AAVV Ostia II. F. BERTI et alii: Ostia II. Le Terme del Nuotatore, scavo dell'ambiente I. Studi miscellanei 16. Roma 1970

= A. Carandini-C. Panella (a cura di) Ostia III. Le Terme del Nuotatore, scavo degli ambienti III, IV, VII. Scavo dell' ambiente V e di un saggio nell'area Sud-Ovest. Studi miscellanei 21. Roma 1973

= A. CARAndini-C. Panella (a cura di) Ostia IV. Le Terme del Nuotatore. Scavo dell' ambiente XVI e dell'area XXV. Studi miscellanei 23. Roma 1977

= Ostia. La taberna dell'Invidioso. Piazzale delle Corporazioni, portico ovest. Saggi sotto il mosaico (a cura di M. Carta-I. Pohl-F. Zevi). NotSc suppl. al vol XXX. Roma 1978

= C. PAVolini: La ceramica comune. Le forme in argilla depurata nell'Antiquarium. Roma 2000

= S. PALLECHI: I mortaria di produzione centro-italiche: corpus dei bolli. Roma 2002

= C. PAnella: Le anfore italiche del II secolo d.C. In: Amphores romaines et histoire èconomique. dix ans de recherche. Col. École Française Rome 114. 1989, 141-156

$=$ H. Patterson-A. Bousquet-H. Di Giuseppe-F. Felici-S. Fontana-R. Witcher-S. Zamponi: Le produzioni ceramiche nella media valle del Tevere tra l'età reppublicana e tardoantica. Acta RCRF 38 (2003) 161-170

= C. PAvolini: Una produzione italica di lucerne: le Vogelkopflampen ad ansa traversata. Boll di Commissione Comunale di Roma 85 (1976-1977) 45-134

= C. Pavolini: Scavi di Ostia XIII. La ceramica comune. Le forme in argilla depurata dell'Antiquarium. Roma 2000

$=\mathrm{J}$. T. PEŇA: Roman period ceramic production in Etruria Tiberina: a geographical and compositional study I-II. Michigan 1987

= S. PETRU: Emonske nekropole (odkrite med leti 1635-1960). Catalogi et monographiae 7. Ljubljana 1972

= LJ. PLESNIČAR GEC: The northern necropolis of Emona. Catalogi et monographiae 8. Ljubljana 1972

= I. Pohl-F. Zevi: Ostia. Casa delle Pareti Gialle. Salone Centrale. Scavo sotto il pavimento a mosaico. NotSc 24 (1970) suppl. 43

= Zs. PongrácZ: Lucerne in: D. Gabler-F. Redő: Scavi nella villa romana di San Potito di Ovindoli (AQ) Rapporto 1989-1990. ActaArchHung 46 (1994) 192

= G. PuCCI: Cumanae testae. La parola del passato. RivStudi Antichi 164, sett-ott. Napoli 1975, 368-371

= G. PUCCI: Terra sigillata italica. Atlante delle forme ceramiche. Ceramica fine romana nel Bacino Mediterraneo (tardo ellenismo e primo impero). Enciclopedia dell'arte antica classica e orientale. Roma 1985, 359-406

= G. PuCCI: L'officina di un ceramista romano. Il territorio di Torrita di Siena nell' antichità. Firenze 1992

= L. QuILICI: Saggi di scavo attorno a Fidenae. NotSc ser.8, anno CCCLXXII, XXX 263-326

= D. Reynolds-D. R. HernandeZ-D. H. ÇONDI: Excavations in the Roman Forum of Buthrotum (Butrint): first to third century pottery assamblages and trade. Acta RCRF 40 (2008) 71-88

= A. RICCI: Per una cronologia delle lucerne tardo repubblicane. Rivista di Studi Liguri 38 (1974) $168-233$

= A. Ricci: Ceramica a pareti sottili. Atlante delle forme ceramiche. Ceramica fine romana nel bacino del Mediterraneo (tardo ellenismo e primo impero) II. Roma 1985, 231-357

= G. RIzzo: Samia etiamnunc in esculendis laudantur (Pl. N.H. XXXV. 160-161) 'vasi aretini' a Roma. MEFR 110 (1998) 799-848

= H. S. Robinson: Pottery of the Roman Period. Chronology. The Athenian Agora V. Princeton N.J. 1959

$=$ S. SANTORO BIANCHI-B. FABBRI: Il contributo delle analisi archeometriche allo studio delle ceramiche grezze e comuni. Il rapporto forma/funzione/impasto. Atti della I giornata di archeometria della ceramica (a cura di S. Santoro Bianchi). Studi e scavi 4. Bologna 1997

= E. SChINDlER-KAudelKA: Die dünnwandige Keramik vom Magdalensberg. Kärntner Museumsschriften 58. Klagenfurt 1975

$=$ E. SCHINDLER-KAUdELKA: Die dünnwandige Gebrauchskeramik vom Magdalensberg.2. Die pareti sottili vom Südhang des Magdalensberg Die Ausgrabungen auf dem Magdalensberg 1980 bis 1986. Klagenfurt 1998, 389-427

= E. SchindLeR-KAudelKa: La ceramica a pareti sottili del Magdalensberg 1975-1998-2011. In: Emona between Aquileia and Pannonia (ed. I. Lazar-B. Zuupanek). Piran 2012, 323-366 
SCHINDLER-SCHEFFENEGGER 1977

Settefinestre 1985

SIMONETT 1941

VEGAS 1963-1964

VEGAS 1968

VEGAS 1973

Vegas-Martín LóPeZ 1982

WARNER SLANE 1987

ZABEHLICKY-SCHEFFENEGGER 1992

ZACCAGNINI 1998

ŽERJAL 2008
= M. SChindLER-S. SCHEFFEnEgGer: Die glatta rote Terra Sigillata vom Magdalensberg. Archäologische Forschungen auf dem Magdalensberg 5. Kärntner Museumsschriften 62. Klagenfurt 1977

= A. RICCI: Settefinestre. Una villa schiavistica nell'Etruria romana 2. La villa e sui reperti. (a cura di A. Carandini-A. Ricci) Modena 1985

$=$ Сн. SimonetT: Tessiner Gräberfelder. Monographien zur Ur-und Frühgeschichte der Schweiz 3. Basel 1941

= M. Vegas: Difusión de algunas formas de vasitos da paredes fines. Acta RCRF 5-6 (1963-64) 61-83

= M. VegAS: Römische Keramik von Gabii (Latium). Bonner Jb 168 (1968) 13-55

= M. Vegas: Cerámica común del Mediterráneo Occidental. Univ.Barcelona. Publ.eventuales 22. Barcelona 1973

= M. Vegas-A. Martín López: Ceramica común y de paredas finas. In: M. Almagro Gorbea: El santuario de Juno en Gabii. Excavaciones 1956-1969. Roma 1982, 451-504

= K. WARNER SLANE: Italian sigillata imports in Corinth. Acta RCRF 35-36 (1987) 189-205

$=\mathrm{S}$. ZABEHLICKY-SCHEFFENEGGER: Terra sigillata tardo padana. Acta RCRF 31-32 (1992) 415-443

= C. ZACCAGNINI: Il thymiaterion nel mondo greco. Analisi, fonti, tipologia, impieghi. Roma 1998

$=$ T. ŽERJAL: Eastern imports in the ager Tergestinus. Acta RCRF 40 (2008) 131-140 